\title{
Mathematical Foundations for Probability and Causality
}

\author{
Glenn Shafer
}

\begin{abstract}
Event trees, and more generally, event spaces, can be used to provide a foundation for mathematical probability that includes a systematic understanding of causality. This foundation justifies the use of statistics in causal investigation and provides a rigorous semantics for causal reasoning.
\end{abstract}

Causal reasoning, always important in applied statistics and increasingly important in artificial intelligence, has never been respectable in mathematical treatments of probability. But, as this article shows, a home can be made for causal reasoning in the very foundations of mathematical probability. The key is to bring the event tree, basic to the thinking of Pascal, Huygens, and other pioneers of probability, back into probability's foundations. An event tree represents the possibilities for the step-by-step evolution of an observer's knowledge. If that observer is nature, then the steps in the tree are causes. If we add branching probabilities, we obtain a probability tree, which can express nature's limited ability to predict the effects of causes.

As a foundation for the statistical investigation of causality, event and probability trees provide a language for causal explanation, which gives rigorous meaning to causal claims and clarifies the relevance of different kinds of evidence to those claims. As a foundation for probability theory, they allow an elementary treatment of martingales,

1991 Mathematics Subject Classification. Primary ; Secondary . 
without distracting and irrelevant technicalities of measure theory. As a foundation for causal reasoning in artificial intelligence, they give rise to dynamic logics that can express expectation as well as certainty and can handle rigorously the task of refining descriptions of causal mechanisms. This article explores the role of event and probability trees in all three areas - the statistical investigation of causality, the foundations of probability, and causal reasoning.

We begin, in Section 1, with a look at how causal relations in finite probability trees imply statistical associations among events and variables, enabling observed associations to serve as evidence for the causal relations. This connection between causality and statistical association is a commonplace, but in the probability-tree setting it acquires, for the first time, a rigorous and detailed expression. Section 2 then explores how a finite event tree can be refined through the addition of more detail and studies the extent to which the causal relations studied in Section 1 are invariant under refinement. Section 3 generalizes the ideas of Section 1 by developing an abstract theory, in which event trees are thought of as partially ordered sets. This abstract approach accommodates infinities and therefore can serve as a foundation for advanced mathematical probability theory. Section 4 shows how probability structure can be added to abstract event trees. This is done using martingales rather than branching probabilities, so that partial probability structures become as natural as complete probability structures. Section 5 analyzes refinement for abstract event trees, and exploits this analysis to develop a new idea-the idea of an event space, in which events and situations defined at different levels of detail can coexist. Event spaces are sufficiently flexible to serve as a semantics for logics that can be used for probabilistic and causal reasoning, and they therefore provide a framework for the unification of probability modeling and artificial intelligence. 
This article grew out of a tutorial on my recent book, The Art of Causal Conjecture (MIT Press, 1996), and a few related articles (Shafer 1995, 1996b, 1996c). In its original design, the article was purely expository. As it has evolved, however, the article has become the vehicle for several new developments. The treatment of the statistics of causality in Section 1 covers only a few of the results on this topic that appear in The Art of Causal Conjecture, but these are developed in a new and more direct way that may be more informative for some readers. Sections 3 and 5, which deals with abstract event trees and their refinement, improve substantially on the analysis in The Art of Causal Conjecture. The work in Section 6 is entirely new.

\section{Using Probability Trees for Causal Explanation}

The idea of a probability tree (an event tree with branching probabilities) can be traced back to the seventeenth-century pioneers of probability theory, especially Christiaan Huygens (see Edwards 1987). In general, a probability tree represents the possibilities for the step-by-step evolution of an observer's knowledge. If that observer is nature, then the steps in the tree represent causes, and the probabilities express nature's limited ability to predict causes and their effects.

In this section, we rely on the simple graph-theoretical idea of a probability tree, as exemplified by Figures 1 and 2, to show how probability trees can be used to define causal relations among events and variables and how evidence for these causal relations can be found in statistical observations. 


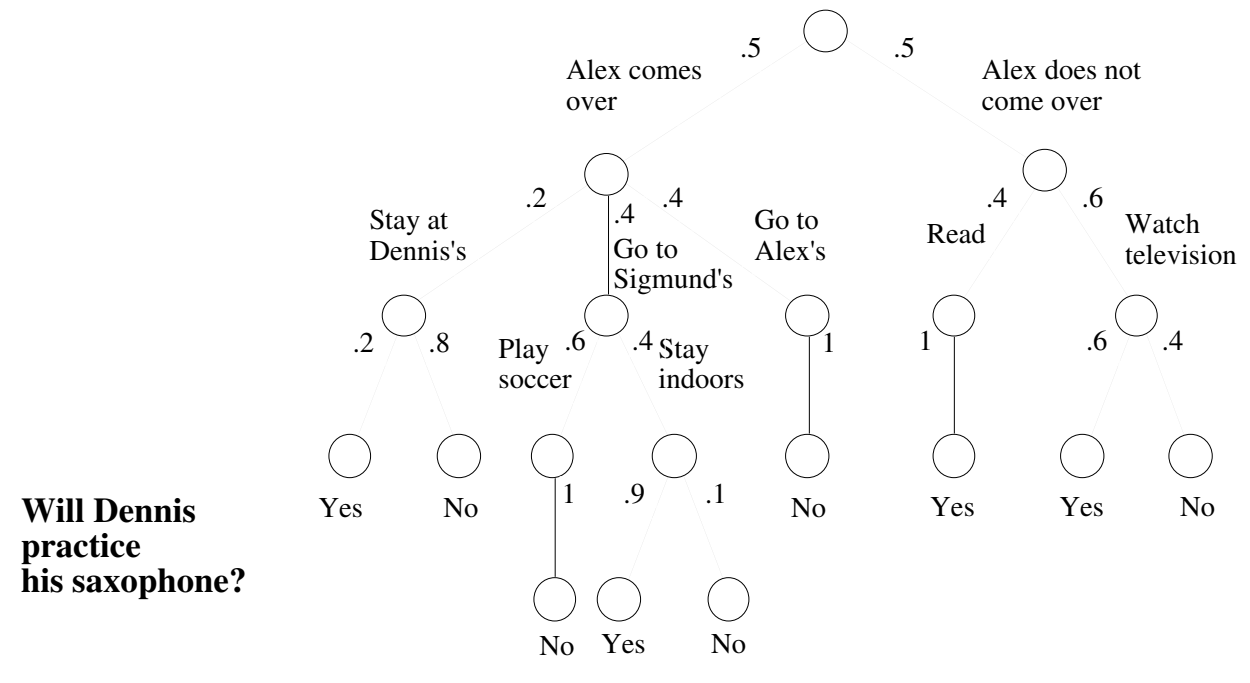

Figure 1 The habits of Dennis and his friends on summer afternoons. The numbers are probabilities for what will happen in each situation (circle).

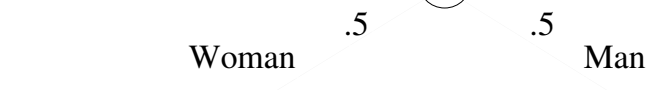

Sex

5

Education

8 yrs

$12 \mathrm{yrs}$

12 yrs

.5

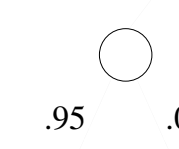

Salary

$\$ 8$

$\$ 12 \$ 8$

.95

.95

.05

.05

.95

$\bigcirc \bigcirc 0$

Figure 2 The norms of an imaginary discriminatory society. This society educates men more than women, but there is some overlap. People are usually paid in proportion to their education, but employers may deviate from proportionality for an exceptionally capable or hapless employee, provided they stay within the range of pay customary for the employee's sex. 
Most people who have 12 years of education are paid $\$ 12$, but a few women are paid less and a few men more. Hence women with 12 years of education are paid less on average than men with the same education. Similarly, most people who are paid $\$ 12$ have 12 years of education, but this group includes a few men with 16 years of education and a few women with only 8 . Hence women who are paid $\$ 12$ have less education on average than men paid the same. ${ }^{1}$

1.1. Causal Talk. Probability trees for nature provide a framework for a variety of causal talk. We can use Figures 1 and 2 to talk about singular causation-why Dennis forgot to practice on a particular occasion or why a particular man is well paid. We can also use them to talk about average causation—what makes Dennis likely to practice or why women are poorly paid on average. And we can use them to distinguish between contingent and structural causes. ${ }^{2}$ A step or sequence of steps in a tree is a contingent cause; Dennis's going to Alex's house is a contingent cause of his forgetting to practice. The habits, preferences, norms, or laws of physics that determine the possibilities and

${ }^{1}$ Situations where discrimination against women is alleged often have similar statistical features. Women are paid less on average than men with the same education but have less education on average than men with the same pay. See Dempster (1988) and Finkelstein and Levin (1990).

${ }^{2}$ The word "cause" is used here in an informal way. It will not used as a technical term in this article. This word has so many legitimate uses (in addition to some questionable ones), and it is so often the object of philosophical debates irrelevant to our purposes, that it would be misleading and unnecessarily contentious to assign it a particular technical meaning. (For a contrasting view, see Kutschera 1993.) 
their probabilities are structural causes; Dennis fails to practice at Alex's house because he prefers to do other things there.

1.2. Probability Talk. Standard probability talk is also easy in a probability tree. The paths through the tree-from the initial situation to the terminal situations-constitute the sample space. Following standard practice, we call subsets of the sample space events, and we call functions on it variables. In Figure 1, the event that Dennis practices is represented by the four paths to terminals situations marked "Yes." In Figure 2, salary is the variable whose value for a path is written next to the last step on the path.

In standard usage, a probability structure is given by a single probability measure on a sample space, but in a probability tree, we have many probability measures, one for each situation in the tree. As Figures 3 and 4 illustrate, each situation has its own probability for every event and its own expected value for every variable. This gives depth to the idea that steps in the tree-steps from one situation to another-are contributing causes. Their influence on events and variables is indicated by changes in the events' probabilities and the variables' expected values. 


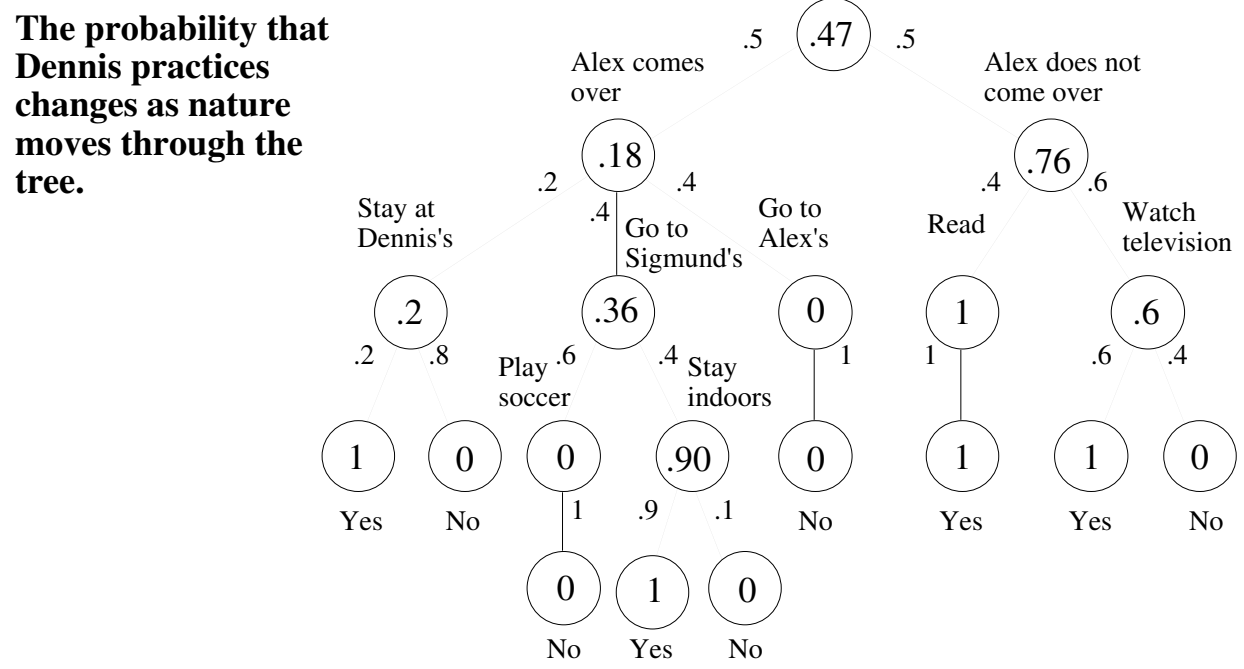

Figure 3 The probability that Dennis practices is shown in each situation.

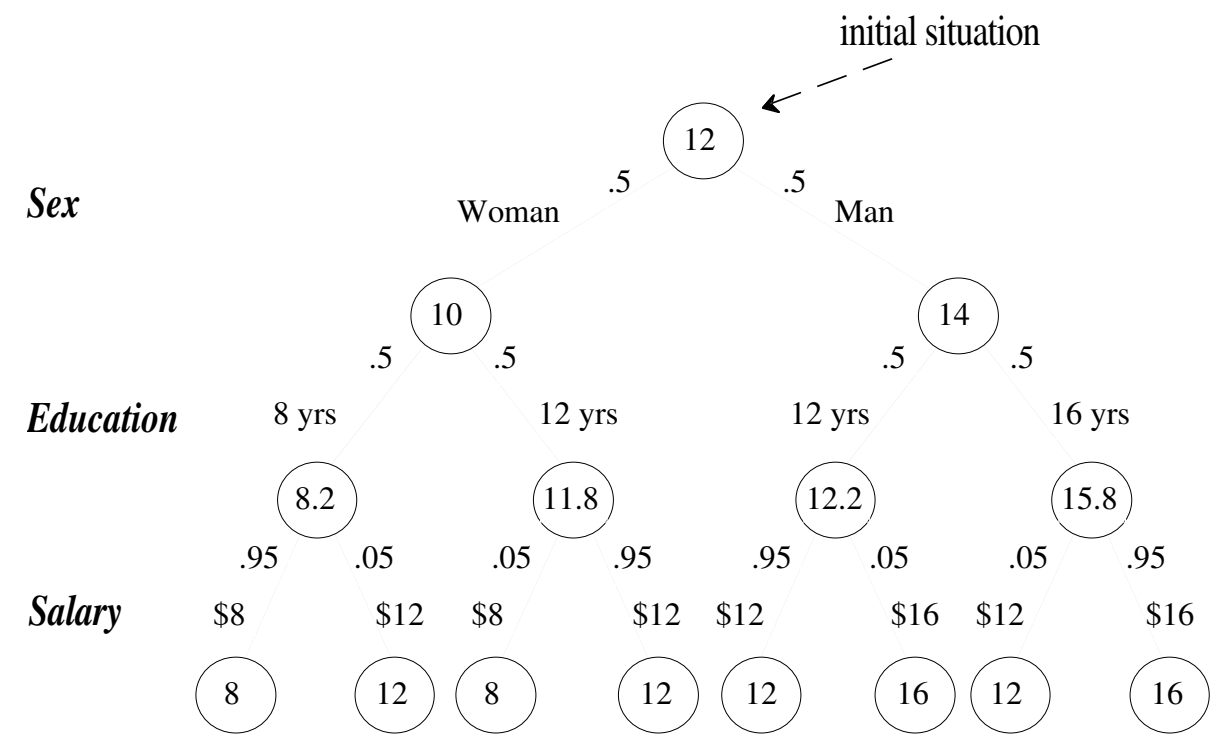

Figure 4 The expected value of salary is shown in each situation.

We will use a notation that makes explicit the dependence of probabilities and expected values on the situation:

- $\mathrm{P}_{\mathrm{S}}(\mathrm{E})$ is the probability of the event $\mathrm{E}$ in $\mathrm{S}$. 
- $\mathrm{E}_{\mathrm{S}}(\mathrm{X})$ is the expected value of the variable $\mathrm{X}$ in the situation $\mathrm{S}: \mathrm{E}_{\mathrm{S}}(\mathrm{X}):=$ $\sum \mathrm{XP}(\mathrm{X}=\mathrm{x})$.

- $\operatorname{Var}_{S}(X)$ is the variance of $X$ in $S: \operatorname{Var}_{S}(X):=E_{S}\left(\left[X-E_{S}(X)\right]^{2}\right)$

- $\operatorname{Cov}_{S}(X, Y)$ is the covariance of $X$ and $Y$ in $S: \operatorname{Cov}_{S}(X):=E_{S}\left(\left[X-E_{S}(X)\right]\left[Y-E_{S}(Y)\right]\right)$ $=\mathrm{E}_{\mathrm{S}}(\mathrm{XY})-\mathrm{E}_{\mathrm{S}}(\mathrm{X}) \mathrm{E}_{\mathrm{S}}(\mathrm{Y})$.

- $\mathrm{P}_{\mathrm{S}}(\mathrm{s})$ is probability of a step $\mathrm{s}$ in the situation $\mathrm{S}$. (If $\mathrm{s}$ is below $\mathrm{S}, \mathrm{P}_{\mathrm{S}}(\mathrm{s})$ is the product of the branching probabilities on all the steps from $\mathrm{S}$ to and including $\mathrm{s}$. If $\mathrm{s}$ is not below $\mathrm{S}, \mathrm{P}_{\mathrm{S}}(\mathrm{s})$ is zero.)

- $\Delta_{\mathrm{S}} \mathrm{X}$ is the change in $\mathrm{X}$ 's expected value on step s. (If $\mathrm{s}$ goes from $\mathrm{S}$ to $\mathrm{T}$, then $\Delta_{\mathrm{S}} \mathrm{X}:=$ $\left.\mathrm{E}_{\mathrm{T}}(\mathrm{X})-\mathrm{E}_{\mathrm{S}}(\mathrm{X}).\right)$

1.3. The Analysis of Variance. Analysis of variance is quite simple in probability trees: we simply apportion $\operatorname{Var}_{S}(X)$, the variance of $X$ in $S$, among the steps of the tree. The portion assigned to step $\mathrm{s}$ is

$$
\mathrm{P}_{\mathrm{S}}(\mathrm{s}) \cdot\left(\Delta_{\mathrm{S}} \mathrm{X}\right)^{2} .
$$

Figure 5 illustrates this decomposition for the example of Figure 4, with $\mathrm{X}$ as salary and $\mathrm{S}$ as the initial situation. 


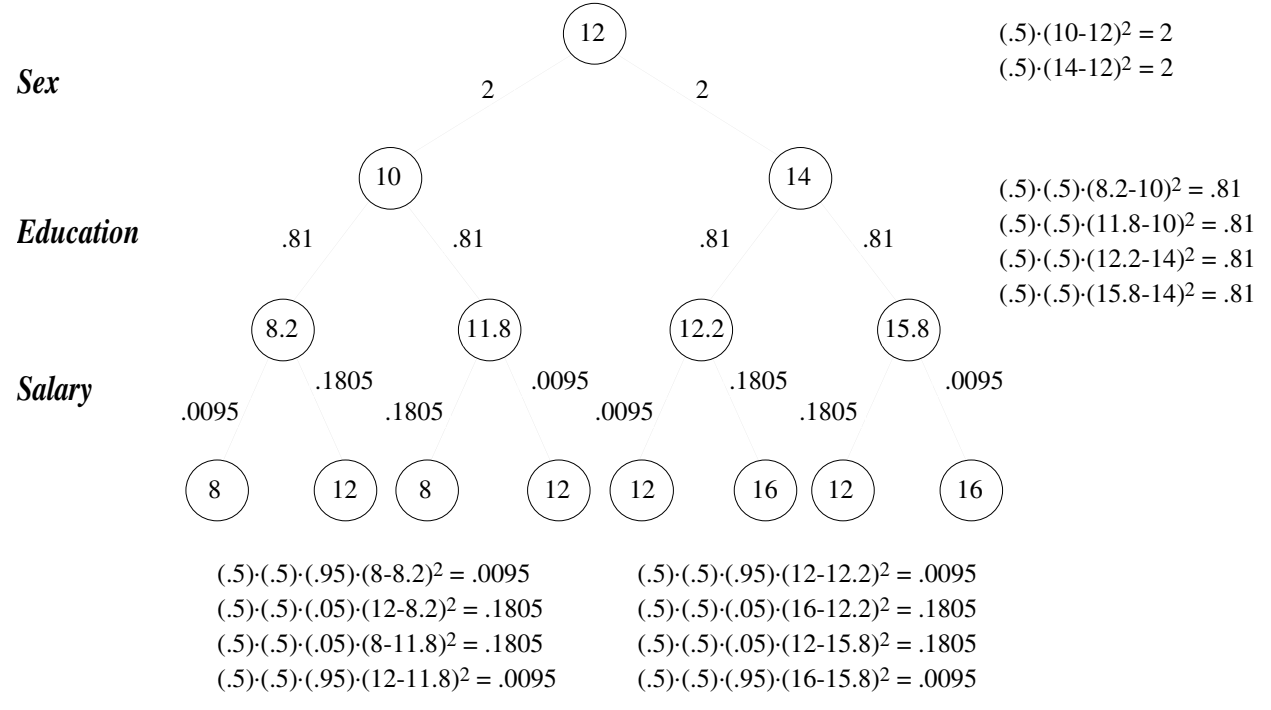

Figure 5 Apportioning the variance for salary. The two steps at which the sex of a child is determined account for $50 \%$ of the initial variance of salary (4 out of 8 units). The four steps at which education is determined account for another $40.5 \%$ (3.24 out of 8 units). The steps at the bottom of the tree account for the remaining $9.5 \%$.

The rule for apportioning variance among the steps of a probability tree generalizes to covariance;

$$
\mathrm{P}_{\mathrm{S}}(\mathrm{s}) \cdot \Delta_{\mathrm{S}} \mathrm{X} \cdot \Delta_{\mathrm{S}} \mathrm{Y}
$$

is the portion of $\operatorname{Cov}_{S}(\mathrm{X}, \mathrm{Y})$ we attribute to the step s. The following proposition establishes that this does indeed define a decomposition.

Proposition 1. Suppose $X$ and $Y$ are variables in a probability tree, and $S$ is a situation in the tree. Then

$$
\operatorname{Cov}_{S}(X, Y)=\sum_{s} P_{S}(s) \cdot \Delta_{S} X \cdot \Delta_{S} Y,
$$

where the sum is over all steps in the tree. 
Proof It suffices to consider the case where $S$ is the initial situation, since any situation is the initial situation in the subtree beginning with it.

In order to prove that (3) holds for the initial situation of every probability tree, we can use induction on the number of nonterminal situations. If there is only one nonterminal situation, as in Figure 6, then (3) reduces to

$$
\operatorname{Cov}_{S}(X, Y)=\sum P_{S}(\omega) \cdot\left[X(\omega)-E_{S}(X)\right] \cdot\left[Y(\omega)-E_{S}(Y)\right],
$$

$\omega$

where the sum is over the elements of the sample space. This is the usual definition of covariance.

In order to complete the proof, consider a probability tree with more than one nonterminal situation, and select a situation $\mathrm{U}$ that is neither terminal nor initial. Write

- $\mathrm{T}$ for the mother of $\mathrm{U}$,

- $\mathrm{V}_{1}, \ldots, \mathrm{V}_{\mathrm{k}}$ for the daughters of $\mathrm{U}$,

- $\mathrm{p}_{0}$ for the probability of $\mathrm{U}$ in $\mathrm{T}$, and

- $\mathrm{p}_{\mathrm{i}}$ for the probability of $\mathrm{V}_{\mathrm{i}}$ in $\mathrm{T}$,

as in Figure 7. If we remove $\mathrm{U}$, making the $\mathrm{V}_{\mathrm{i}}$ daughters of $\mathrm{T}$, then we obtain the tree on the right of Figure 7, which has one less nonterminal situation. This smaller tree has the same terminal situations and hence the same variables as the original tree, and when we assign probability $p_{0} p_{i}$ to $V_{i}$ in $T$, these variables have the same expected values in all the situations that remain. By the inductive hypothesis, (3) holds for this smaller tree. Hence it suffices to show that the sum on the right-hand side of (3) is the same for the smaller as for the larger tree. But when we reduce the larger tree to the smaller tree, the sum changes only inasmuch as

$$
\mathrm{qp}_{0} \Delta \mathrm{X} \Delta \mathrm{Y}+\mathrm{qp}_{0} \sum_{\mathrm{i}=1}^{\mathrm{k}} \mathrm{p}_{\mathrm{i}} \Delta_{\mathrm{i}} \mathrm{X} \Delta_{\mathrm{i}} \mathrm{Y}
$$


is replaced by

$$
\mathrm{qp}_{0} \sum_{\mathrm{i}=1}^{\mathrm{k}} \mathrm{p}_{\mathrm{i}}\left[\Delta \mathrm{X}+\Delta_{\mathrm{i}} \mathrm{X}\right]\left[\Delta \mathrm{Y}+\Delta_{\mathrm{i}} \mathrm{Y}\right]
$$

where $\mathrm{q}$ is the probability of $\mathrm{T}$ in the initial situation, $\Delta \mathrm{X}:=\mathrm{E}_{\mathrm{U}}(\mathrm{X})-\mathrm{E}_{\mathrm{T}}(\mathrm{X})$, and $\Delta_{\mathrm{i}} \mathrm{X}:=$ $\mathrm{E}_{\mathrm{V}_{\mathrm{i}}}(\mathrm{X})-\mathrm{E}_{\mathrm{U}}(\mathrm{X})$. Multiplying the binomials in (5), we obtain

$$
\mathrm{qp}_{0} \Delta \mathrm{X} \Delta \mathrm{Y}+\mathrm{qp}_{0} \sum_{\mathrm{i}=1}^{\mathrm{k}} \mathrm{p}_{\mathrm{i}} \Delta_{\mathrm{i}} \mathrm{X} \Delta_{\mathrm{i}} \mathrm{Y}+\mathrm{qp}_{0} \Delta \mathrm{Y} \sum_{\mathrm{i}=1}^{\mathrm{k}} \mathrm{p}_{\mathrm{i}} \Delta_{\mathrm{i}} \mathrm{X}+\mathrm{qp}_{0} \Delta \mathrm{X} \sum_{\mathrm{i}=1}^{\mathrm{k}} \mathrm{p}_{\mathrm{i}} \Delta_{\mathrm{i}} \mathrm{Y}
$$

And the last two terms are zero, because

$$
\mathrm{E}_{\mathrm{U}}(\mathrm{X})=\sum_{\mathrm{i}=1}^{\mathrm{k}} \mathrm{p}_{\mathrm{i}} \mathrm{E}_{\mathrm{V}_{\mathrm{i}}}(\mathrm{X}) \text { and } \mathrm{E}_{\mathrm{U}}(\mathrm{Y})=\sum_{\mathrm{i}=1}^{\mathrm{k}} \mathrm{p}_{\mathrm{i}} \mathrm{E}_{\mathrm{V}_{\mathrm{i}}}(\mathrm{Y})
$$

It should be emphasized that Equation (3) is a decomposition, not a representation of $\operatorname{Cov}_{\mathrm{S}}(\mathrm{X}, \mathrm{Y})$ as a weighted average. The right-hand side is not a weighted average of the products $\Delta_{\mathrm{S}} \mathrm{X} \cdot \Delta_{\mathrm{S}} \mathrm{Y}$, because the "weights" $\mathrm{P}_{\mathrm{S}}(\mathrm{s})$ do not add to one.

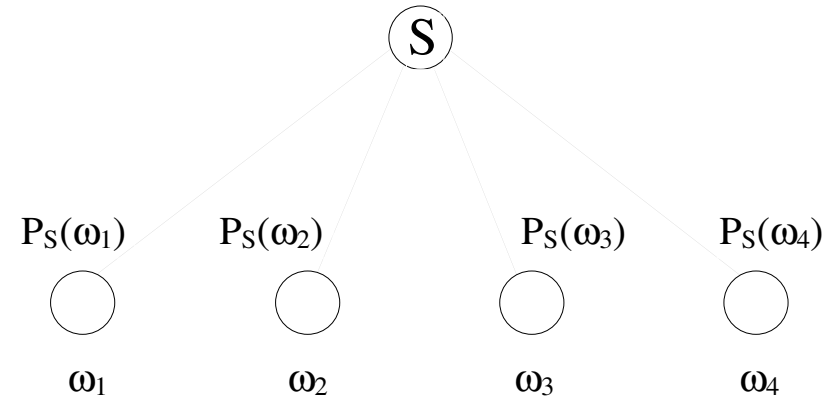

Figure 6 When there is only one nonterminal situation, the steps constitute the sample space, and the branching probabilities are the same as the probabilities of the elements of the sample space in the initial situation. 


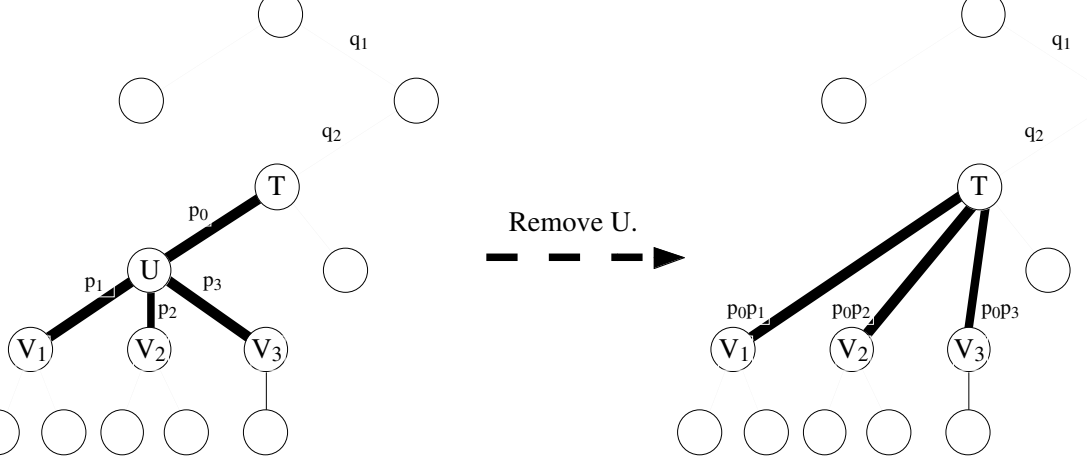

Figure 7 The terms in (4) are the portions of the covariance associated with the four steps marked on the left, while the terms in (5) are the portions associated with the three steps marked on the right. In this example, $\mathrm{q}=\mathrm{q}_{1} \mathrm{q}_{2}$.

1.4. Causal Conjecture. The rules for decomposing variance and covariance are rich in implications. Here are a few of them:

Proposition 2. Suppose $X$ and $Y$ are variables in a probability tree.

1. If there is no step in the tree where $X$ and $Y$ both change in expected value, then $X$ and $Y$ are uncorrelated in every situation $\left(E_{S}(X Y)=E_{S}(X) E_{S}(Y)\right.$ for every $\left.S\right)$.

2. If there is no step in the tree where the expected value of $X$ goes up while the expected value of $Y$ goes down, and vice versa, then their correlation is nonnegative in every situation $\left(E_{S}(X Y) \geq E_{S}(X) E_{S}(Y)\right.$ for every $\left.S\right)$.

3. If there is no step in the tree where $X$ and $Y$ both change in probability, then $X$ and $Y$ are independent in every situation $\left(P_{S}(X=x \& Y=y)=P_{S}(X=x) P_{S}(Y=y)\right.$ for every $x, y$, and $S$ ).

Proof If $\mathrm{X}$ and $\mathrm{Y}$ never change in expected value on the same step, then $\Delta_{\mathrm{S}} \mathrm{X} \Delta_{\mathrm{S}} \mathrm{Y}$ is zero for every step s. It follows from (3) that for every situation $\mathrm{S}, \operatorname{Cov}_{\mathrm{S}}(\mathrm{X}, \mathrm{Y})$ is zero, or $E_{S}(X Y)=E_{S}(X) E_{S}(Y)$ 
Similarly, if $\Delta_{\mathrm{S}} \mathrm{X}$ and $\Delta_{\mathrm{S}} \mathrm{Y}$ never have opposite signs, then their product is always nonnegative, and it then follows from (3) that for every $S, \operatorname{Cov}_{S}(X, Y) \geq 0$, or $E_{S}(X Y) \geq$ $\mathrm{E}_{\mathrm{S}}(\mathrm{X}) \mathrm{E}_{\mathrm{S}}(\mathrm{Y})$.

Statement 3 follows from Statement 1, because the probability that $\mathrm{X}$ has a particular value is the expected value of the variable that is equal to one when $\mathrm{X}$ takes that value and zero otherwise.

These statements illustrate the causal explanation of statistical patterns. If we observe that $\mathrm{X}$ and $\mathrm{Y}$ are uncorrelated in many different situations, then we may conjecture that they have no common causes - there are no steps in nature's tree that affect them both. If we observe that they are positively correlated in many different situations, then we may conjecture that they consistently have common causes - the events that raise or lower the expected level of one of them also raise or lower the expected level of the other.

We say that $\mathrm{X}$ and $\mathrm{Y}$ are causally uncorrelated when there is no step in nature's tree where they both change in expected value. Thus Statement 1 of Proposition 2 says that causally uncorrelated variables are statistically uncorrelated. Similarly, we say that X and $\mathrm{Y}$ are causally independent when there is no step in nature's tree where they both change in probability. Statement 3 says that causally independent variables are statistically independent.

We can also provide a causal interpretation for regression coefficients. According to standard theory, the coefficient of $\mathrm{X}$ in the linear regression of $\mathrm{Y}$ on $\mathrm{X}$ is

$$
\frac{\operatorname{Cov}(\mathrm{X}, \mathrm{Y})}{\operatorname{Var}(\mathrm{X})} \text {. }
$$

The following proposition reveals that this coefficient is a weighted average of the relative changes in Y's and X's expected values on the different steps in nature's tree. 
Proposition 3. Suppose $X$ and $Y$ are variables in a probability tree, and $S$ is a situation in the tree. Then the regression coefficient of $Y$ on $X$ in $S, \operatorname{Cov}_{S}(X, Y) / \operatorname{Vars}_{S}(X)$, is a weighted average, over all the steps in the tree where $X$ changes in expected value, of the ratio of Y's change in expected value to X's. The weight of a step s is the probability of $s$ in S multiplied by the square of X's change in expected value on s. In symbols:

$$
\frac{\operatorname{Cov}_{S}(X, Y)}{\operatorname{Var}_{S}(X)}=\frac{s \text { with } \Delta_{S} X \neq 0}{s \text { with } \Delta_{S} X \neq 0} \frac{\frac{\Delta_{S} Y}{\Delta_{S} X} P_{S}(s) \cdot\left(\Delta_{S} X\right)^{2}}{\left.P_{S} X\right)^{2}}
$$

Proof Equation (6) follows immediately from (3) when we write its right-hand side in the form

$$
\frac{\mathrm{P}_{\mathrm{S}}(\mathrm{s}) \cdot \Delta_{\mathrm{S}} \mathrm{Y} \cdot \Delta_{\mathrm{S}} \mathrm{X}}{\mathrm{s}_{\mathrm{S}}(\mathrm{s}) \cdot\left(\Delta_{\mathrm{S}} \mathrm{X}\right)^{2}} .
$$

If the ratios $\Delta_{\mathrm{S}} \mathrm{Y} / \Delta_{\mathrm{S}} \mathrm{X}$ that the regression coefficient averages are quite disparate, then the average is not very interesting, and we will say that the regression coefficient is causally meaningless; it has no simple causal interpretation. But if these ratios are quite similar, then we will feel that the average does have causal meaning. The most interesting case is where the ratios are all equal. (Figure 8 gives an example.) In this case, the regression coefficient will be the same in every situation, and we will say that there is a stable causal relation between $\mathrm{X}$ and $\mathrm{Y}$. This relation deserves a name; I have proposed that we call $\mathrm{X}$ a linear sign of $\mathrm{Y}$. The concept of linear sign is much more general than the concepts of linear causality usually considered by statisticians and philosophers (e.g., Freedman 1991, Humphreys 1989). 


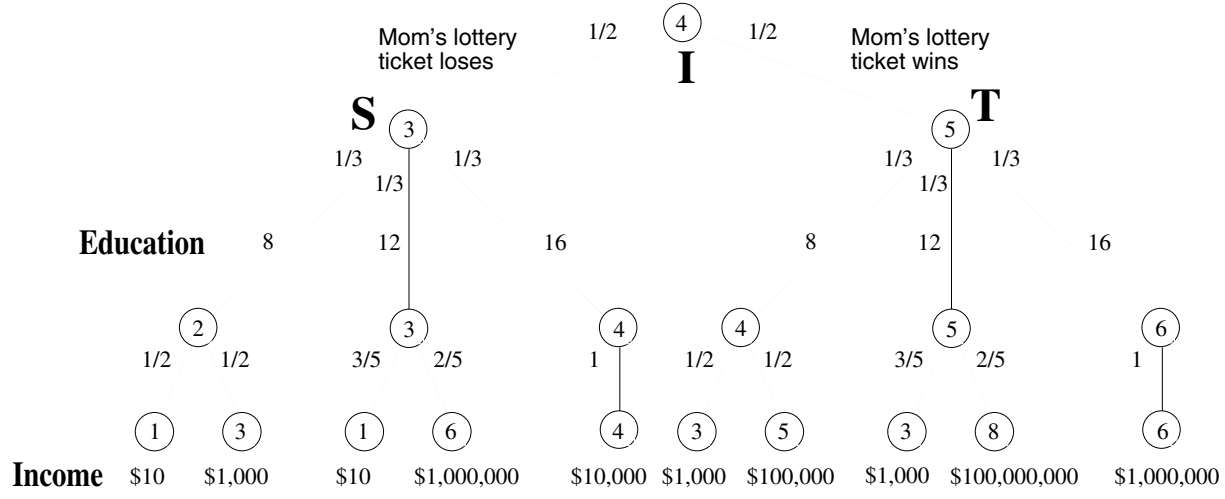

The expected value of $\log _{10}$ (Income) is shown in each situation.

Figure 8 In this society, a person's expected income goes up when the person gets more education. In fact, when the expected value of Education changes, the expected value of the logarithm (to the base 10) of Income changes by exactly one-fourth as much. Consequently, the coefficient of Education in the linear regression of $\log _{10}$ (Income) on Education is $1 / 4$ in every situation where Education is not yet settled. The regression is $1+(1 / 4)$ Education in the initial situation I, (1/4)Education in S, and 2+(1/4)Education in T.

Figure 9 summarizes our understanding of causality and statistical observation. Correlation does not prove causality; we cannot deduce a causal relation from a statistical association. But causality does imply statistical association, and hence statistical association can give us grounds for conjecturing a causal relation. Causality can explain correlation. 


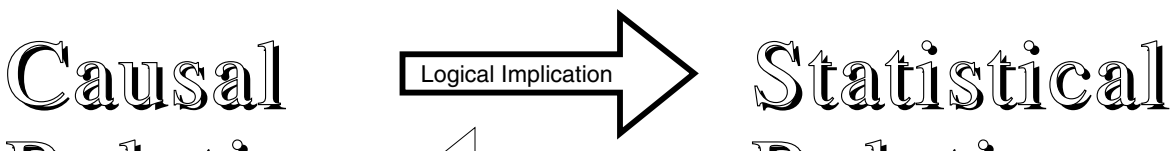 Relation conotur Relation}

\section{Examples}

$\mathrm{X}$ and $\mathrm{Y}$ are causally
uncorrelated.
$\begin{aligned} & \mathrm{X} \text { and } \mathrm{Y} \text { are causally } \\ & \text { independent. }\end{aligned}$

Figure 9 The dual connection between causal and statistical relations. More examples are given in The Art of Causal Conjecture.

\section{Refinement}

Probability trees often seem naive, especially as part of a causal discussion, simply because they necessarily leave aside many issues and factors that might be brought into the discussion. The probability tree in Figure 1, for example, says nothing the factors that decide whether Alex will come to Dennis's house on a particular afternoon. How can the tree be meaningful and valid if these factors are not taken into account?

The answer is that additional factors can be taken into account without falsifying the assertions made in a particular probability tree. In other words, we can construct a more refined tree that does take more into account but agrees with the assertions already made 
in the original tree. In order to make a convincing case for probability trees, we obviously need to show that such refinement really is possible. This is the purpose of this section.

This section works is the same finite, graph-theoretical context as the preceding section. In this context, as we will see, any refinement is a composition of elementary refinements, each of which involve interpolation below a single situation. In the next section, we will study refinement in the more general context of abstract event trees.

Once we have understood the idea of refinement, we will need to look again at the causal concepts of the preceding section. As we will see, they need to be strengthened slightly in order to for us to be sure that they will persist when a probability tree is refined.

2.1. Elementary Refinements. When we suppose that a given probability tree represents nature's limited ability to predict, we are not supposing that nature is blindfolded while each step is taken. When we suppose, for example, that nature gives even odds on whether a woman will complete 12 years of education or only 8 , we are supposing only that these are nature's odds at the moment when the woman is conceived (and her sex is determined). As Figure 10 illustrates, nature's odds on whether the woman eventually chooses more education may change in the course of the woman's childhood. 


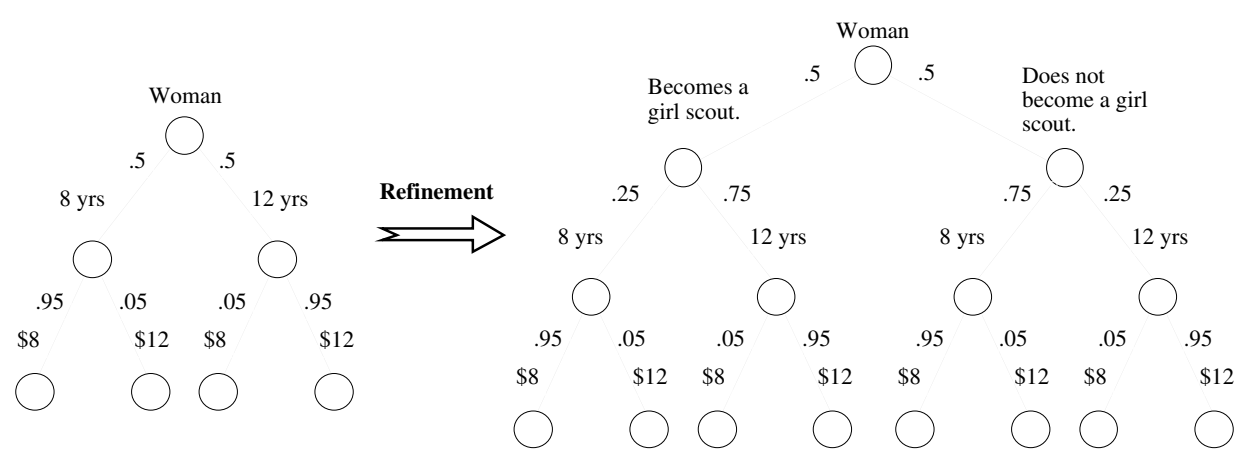

Figure 10 More detail on how the educational level of a woman is determined in the society of Figure 2. The more refined tree does not contradict any causal assertions in the original tree. It continues to assert, for example, that a woman with eight years of education has only a $5 \%$ chance of earning $\$ 12$.

We say that the probability tree on the right in Figure 10 is an elementary refinement of the probability tree on the left. An elementary refinement involves interpolating a new probability tree between a particular situation $\mathrm{S}$ and its daughters. The situation $\mathrm{S}$ may be the initial situation, as in Figure 10, a later situation (as when the tree on the left of Figure 10 is considered as a subtree of Figure 2), or even a terminal situation (in this case, we may prefer to say that we are adding a new tree below $\mathrm{S}$ rather than interpolating a tree between $\mathrm{S}$ and its daughters). As Figure 11 illustrates, the interpolation proceeds as follows:

1. If $\mathrm{S}$ is not terminal in the tree to be refined, remove the subtree below $\mathrm{S}$.

2. Construct a new subtree below $\mathrm{S}$.

3. If $\mathrm{S}$ was originally terminal, stop.

4. If $\mathrm{S}$ was not originally terminal, assign each terminal situation in the new subtree to one of the original daughters of $\mathrm{S}$, taking care to assign at least one to each original 
daughter $\mathrm{T}$, and taking care that the probabilities in $\mathrm{S}$ of the terminal situations assigned to T add up to T's usual probability in $\mathrm{S}$.

5. Then add to each terminal situation in the new subtree the subtree that followed the corresponding daughter of $\mathrm{S}$ in the original tree. (In the example, this takes us from the tree on the right in Figure 11 back to the tree on the right in Figure 10.)

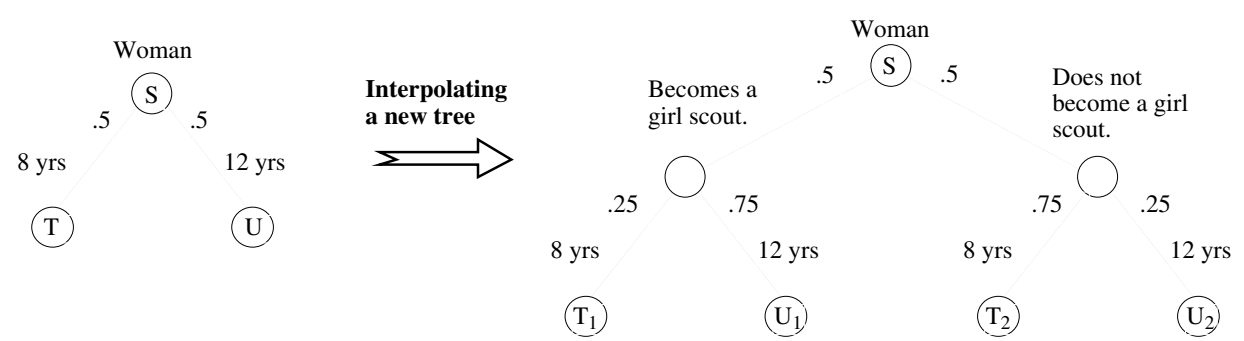

Figure 11 The daughters $T_{1}$ and $T_{2}$ of $S$ are assigned to $T$, while the daughters $U_{1}$ and $\mathrm{U}_{2}$ are assigned to $\mathrm{U}$. The total probability of $\mathrm{T}_{1}$ and $\mathrm{T}_{2}$ in $\mathrm{S}$ is $(.5)(.25)+(.5)(.75)$, or .5 , which was T's probability in S. Similarly, the probabilities of $U_{1}$ and $U_{2}$ add to the probability of $\mathrm{U}$.

Figure 12 shows the elementary refinement of Figure 10 with labels for all the situations in the refined tree that correspond to situations in the initial tree. The two unlabeled situations in the refined tree are not represented in the initial tree; they come into the story only when it is elaborated. Aside from S, all the labeled situations in the refined tree are more detailed versions of situations already present in the initial tree. Situations $\mathrm{T}_{1}$ and $\mathrm{T}_{2}$, for example, are different ways of adding detail to $\mathrm{T}$. This means that $T_{1}$ and $T_{2}$ agree with everything that $T$ already says, including what it says about possibilities and probabilities for future events. We say that $\mathrm{T}_{1}$ and $\mathrm{T}_{2}$ refine $\mathrm{T}$. 


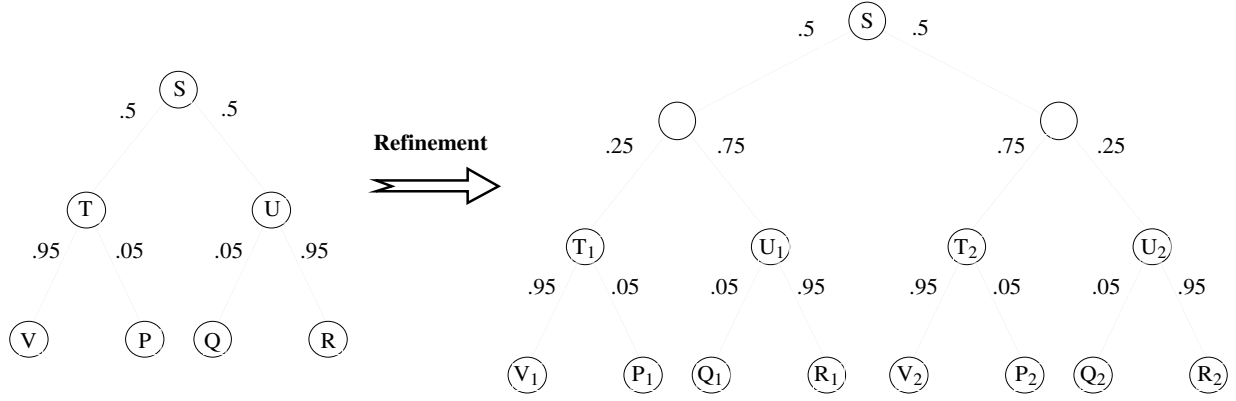

Figure 12 There are two situations in the more refined tree that are not represented in the initial tree.

2.2. Combining Elementary Refinements. We say that one probability tree is a refinement of another if it can be obtained by a sequence of elementary refinements. Figure 13 shows a refinement that can be obtained by combining two elementary refinements. In this example, the refinement does not provide more detail for situations already present in the initial probability tree; it merely adds some new situations.

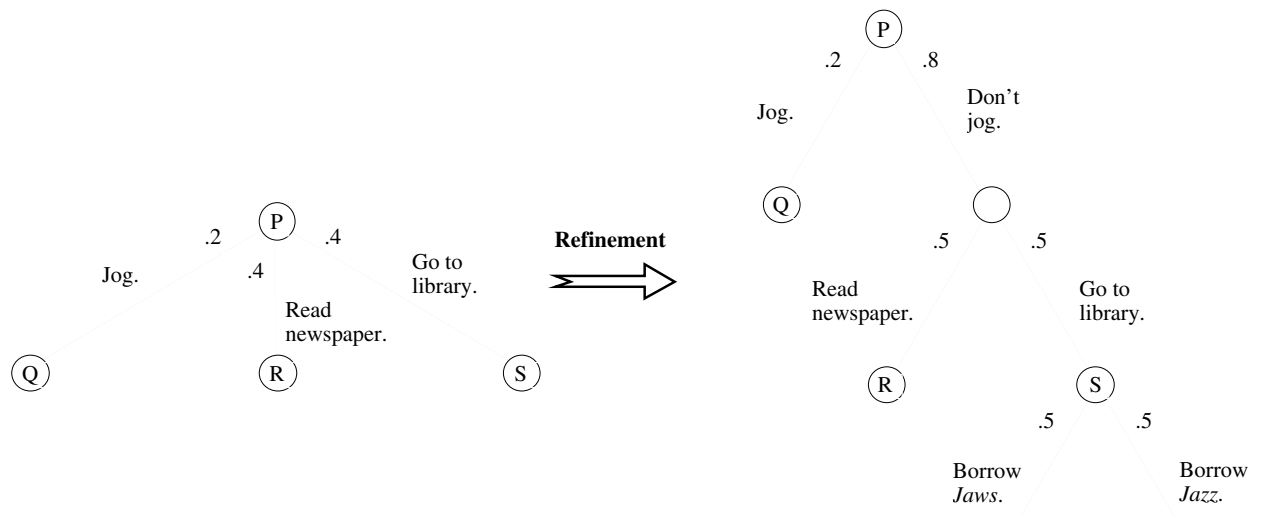

Figure 13 The refinement reveals that the decision whether to jog is taken before a choice is made between reading the newspaper and going to the library. It also shows what can happen at the library. 
2.3. Causal Relations Under Refinement. If the concepts that we defined in the preceding section—causal uncorrelatedness, causal independence, and linear sign—are really causal concepts, then they should not be affected by refinement of nature's tree. If we declare that two variables are causally uncorrelated (have no common causes) because we see that they do not change in expected value on the same step of nature's tree, then we do not want to see this judgment contradicted when we take a closer look at nature's tree.

Unfortunately, the concepts as we have defined them do not quite have this invariance under refinement. Figures 14 and 15 show the difficulty. In Figure 14, the events F and $\mathrm{G}$ appear to be causally independent, for there is no step where they both change in probability. But the refinement in Figure 15 shows that there are in fact steps where they both change in probability.

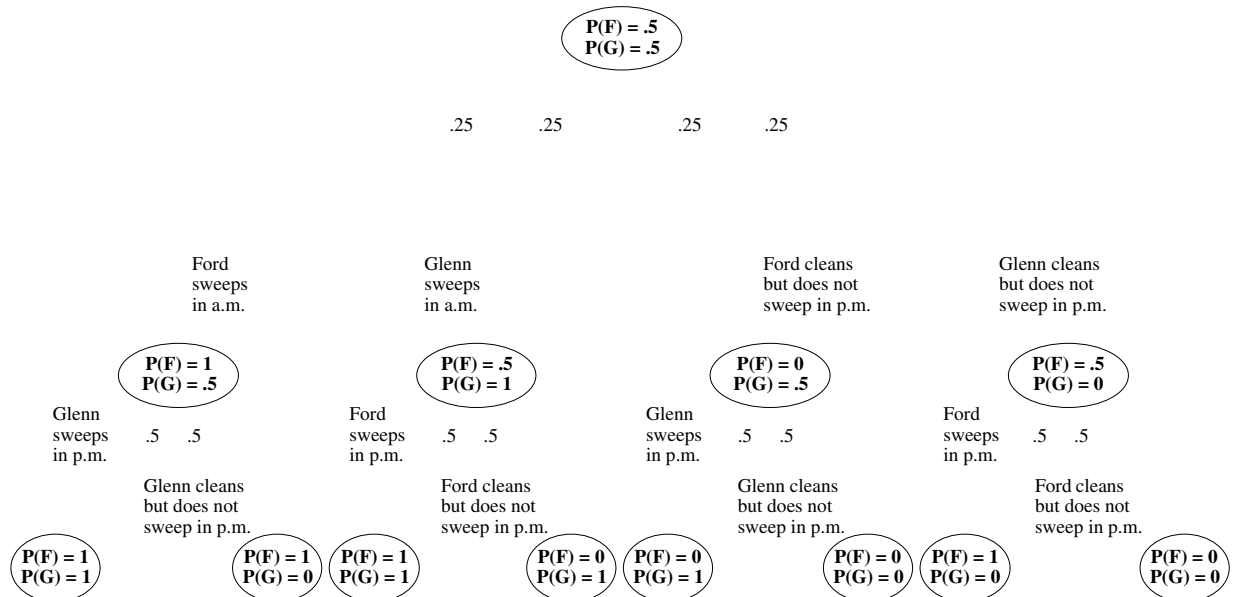

Figure 14 Ford and Glenn clean their room twice a day. One of them cleans in the morning; the other cleans in the afternoon. Sometimes cleaning requires sweeping, sometimes not. We write $\mathrm{F}$ for the event that Ford eventually sweeps the room, and $\mathrm{G}$ for the event that Glenn does. The probabilities of these events are shown for each situation. (Alternatively, F and $\mathrm{G}$ can be thought of as variables, eventually taking the values zero 
or one, and the numbers in the situations are then their expected values.) According to the definition given in Section 1, the events $\mathrm{F}$ and $\mathrm{G}$ are causally independent, because their probabilities do not change on the same step of the tree. (Equivalently, the variables are uncorrelated, because their expected values do not change on the same step.)

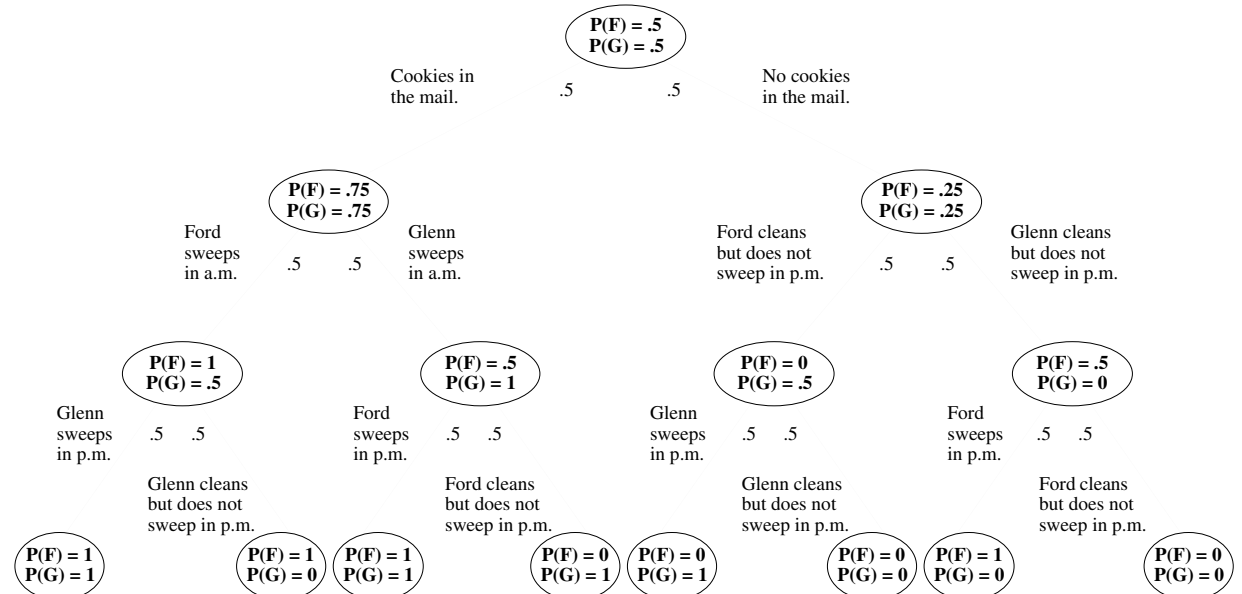

Figure 15 This refinement of Figure 14 reveals that when cookies come in the mail, the probability of sweeping increases in both the morning and afternoon cleanings. The refinement also shows that $\mathrm{F}$ and $\mathrm{G}$ are not really causally independent, for the step in the tree where cookies come increases the probability of both.

The source of the difficulty in this example is clear. It is true that there are no steps in Figure 14 where both events change in probability, but there are closely related steps-alternative steps-where they do. The step from the initial situation in which Ford sweeps in the morning does not change the probability of Glenn's eventually sweeping, but it has a sister that does. We obvious remedy is to rule this out.

Let us say that a situation in a probability tree influences a variable if the variable changes in expected value on at least one of the possible steps immediately after that situation. And let us then revise our earlier definitions as follows: 
- $\mathrm{X}$ and $\mathrm{Y}$ are causally uncorrelated if in a sufficiently refined version of nature's probability tree, there is no situation that influences both $\mathrm{X}$ and $\mathrm{Y}$.

- $\mathrm{X}$ and $\mathrm{Y}$ are causally independent if all functions of $\mathrm{X}$ are causally uncorrelated with all functions of $\mathrm{Y}$.

- $\mathrm{X}$ is a linear sign of $\mathrm{Y}$ with sign coefficient $\mathrm{b}$ if $\mathrm{X}$ and $\mathrm{Y}-\mathrm{bX}$ are causally uncorrelated.

We leave it to the reader to verify that these definitions require more than the earlier definitions and that when they are satisfied by nature's tree at one level of refinement, they will be satisfied by nature's tree at any greater level of refinement.

\section{Abstract Event Trees}

So far, our discussion has been based on a graph-theoretical conception of a tree. Trees are rooted directed graphs without cycles. (For simplicity, we have put the root at the top of our trees, directed all the arrows downward, and suppressed the arrowheads.) This conception is useful. We can draw small trees of this kind, and even large ones have straightforward computational representations. The intellectual tradition of probability is hardly limited, however, to the finite and discrete. So we need to explore more general ways of understanding probability trees. The first step, which we take in this section, is to think abstractly about event trees. In later sections, we will look at refinement, expectation, and probability in abstract event trees and at the more general concept of an event space. 
3.1. Event Trees Defined Abstractly. Abstractly, a tree is a special kind of partially ordered set. (See, for example, Jech 1978.) In this spirit, let us say that an event tree is a set $\Gamma$ with a partial ordering ${ }^{3}$ precedes that has two properties:

Axiom T0 $\Gamma$ has an element I such that I precedes $\mathrm{S}$ for all $\mathrm{S}$ in $\Gamma$.

Axiom T1 If $\mathrm{S}$ precedes $\mathrm{U}$ and $\mathrm{T}$ precedes $\mathrm{U}$, then $\mathrm{S}$ precedes $\mathrm{T}$ or $\mathrm{T}$ precedes $\mathrm{S}$.

We call the elements of an event tree situations. If $\mathrm{S}$ precedes $\mathrm{T}$, we say that $\mathrm{S}$ precedes or is a predecessor of $\mathrm{T}$, and that $\mathrm{T}$ follows or is a successor of $\mathrm{S}$. If $\mathrm{S}$ precedes $\mathrm{T}$ and $\mathrm{S} \neq \mathrm{T}$, we say $\mathrm{S}$ is a strict predecessor and $\mathrm{T}$ is a strict successor. If $\mathrm{S}$ precedes $\mathrm{T}$ or $\mathrm{T}$ precedes $\mathrm{S}$, we say $\mathrm{S}$ and $\mathrm{T}$ are sequenced. Thus Axiom $\mathrm{T} 1$ says that situations with a common successor are sequenced.

The situation I mentioned in Axiom T0 is, of course, unique. We call it $\Gamma$ 's initial situation.

As we will see more clearly shortly, precedes carries a double meaning: $S$ precedes $T$ means that

- T cannot happen unless $\mathrm{S}$ happens earlier (or at the same time, in which case $\mathrm{S}=\mathrm{T}$ ), and

- $\mathrm{T}$ is possible (but has not already happened strictly earlier) whenever $\mathrm{S}$ happens.

${ }^{3}$ Recall that a binary relation is a partial ordering if it is reflexive, asymmetric, and irreflexive. A binary relation is $\mathbf{r}$ is reflexive if $\mathrm{S} \mathbf{r} \mathrm{S}$ for all $\mathrm{S}$, irreflexive if $\mathrm{S} \mathbf{r} \mathrm{S}$ for no $\mathrm{S}$, symmetric if $\mathrm{S} \mathbf{r} \mathrm{T}$ implies $\mathrm{T} \mathbf{r} \mathrm{S}$, asymmetric if $\mathrm{S} \mathbf{r} \mathrm{T}$ and $\mathrm{T} \mathbf{r} \mathrm{S}$ implies $\mathrm{S}=\mathrm{T}$, and transitive if $\mathrm{S} \mathbf{r} \mathrm{T}$ and $\mathrm{T} \mathbf{r} \mathrm{U}$ implies $\mathrm{S} \mathbf{r} \mathrm{U}$. 
More succinctly: $\mathrm{S}$ precedes $\mathrm{T}$ means that $\mathrm{S}$ is a prerequisite for $\mathrm{T}$ and allows $\mathrm{T}$. Here we are understanding $\mathrm{S}$ and T not only as situations but also as instantaneous events. The instantaneous event $\mathrm{S}$ is the event that happens when and only when the event-tree observer arrives in the situation $\mathrm{S}$.

The following proposition shows that our abstract definition of an event tree is consistent with the graph-theoretic conception.

\section{Proposition 4.}

1. Suppose (T,precedes) is a partially ordered set satisfying Axioms T0 and T1, and suppose $\Gamma$ is finite. Then the Hasse diagram of ( $\Gamma$,precedes) is a finite directed tree, with the initial situation as its root. (Figure 16 explains what is meant by the Hasse diagram of a partially ordered set.)

2. Conversely, if we begin with a finite tree drawn as in the preceding sections, write $\Gamma$ for its set of situations, write I for the situation at the top, and write $S$ precedes $T$ whenever there is chain of steps downward from $S$ to $T$, then ( $\Gamma$,precedes) is a partially ordered set satisfying axioms TO and T1.

Statement 1 may fail when $\Gamma$ is infinite, because in this case, the journey from a situation $\mathrm{S}$ to a later situation $\mathrm{T}$ may fail to consist of discrete steps. For example, if we conceive of a different situation for each instant of time between $\mathrm{S}$ and $\mathrm{T}$, then a step can always be divided into smaller steps. 


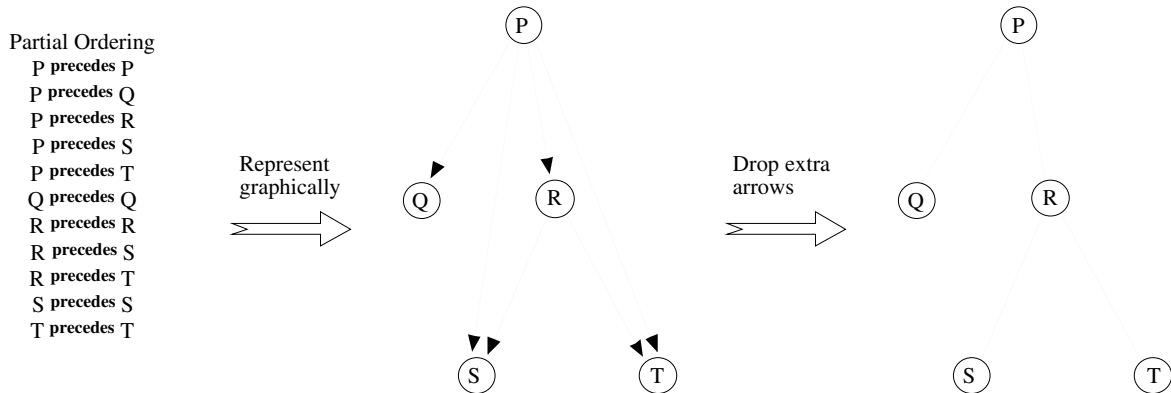

Figure 16 The Hasse diagram for a finite event tree. In general, to obtain the Hasse diagram of a finite partially ordered set ( $\Gamma$,precedes), we draw a graph with the elements of $\Gamma$ as nodes and an arrow from $\mathrm{S}$ to $\mathrm{T}$ whenever $\mathrm{S}$ precedes $\mathrm{T}$, and then we delete the arrow from $\mathrm{S}$ to $\mathrm{T}$ if there is a longer chain of arrows from $\mathrm{S}$ to $\mathrm{T}$. (In the figure, we have also deleted all arrowheads, on the understanding that steps are interpreted as arrows pointing downwards.)

Here, as a counterpoint, is a simple and familiar example of an infinite event tree. Let $\Gamma$ be the set of all continuous real-valued functions that have closed intervals of real numbers beginning at zero as domains and give the value zero to zero. In other words, each element of $\Gamma$ is a function $S$ on some closed interval of the form $[0, \tau]$, with $S(0)=0$. Define the partial ordering precedes on $\Gamma$ by saying that $S$ precedes $T$ if $T$ extends $S-$ i.e., if $\mathrm{S}$ is a function on $\left[0, \tau_{1}\right], \mathrm{T}$ is a function on $\left[0, \tau_{2}\right], \tau_{1} \leq \tau_{2}$, and $\mathrm{T}(\mathrm{t})=\mathrm{S}(\mathrm{t})$ for all $\mathrm{t}$ from 0 to $\tau_{1}$, inclusive. The initial situation is the function that has only zero in its domain and gives it the value zero.

3.2. Paths and the Sample Space. A path in an event tree is a maximal set of sequenced situations - a set $\omega$ of situations such that any two elements of $\omega$ are sequenced, and no other situation in the event tree is sequenced with them all. Every path contains the initial situation. When a path contains a situation, it contains all the 
situation's predecessors. In a finite event tree, every path contains exactly one terminal situation, and every terminal situation is in exactly one path. When a situation is in a path, we say it goes through the path.

We speak also of paths from a particular situation: a path from $\mathrm{S}$ is a maximal set of sequenced successors of S. Thus a path is the same as a path from the initial situation. When $\mathrm{S}$ precedes $\mathrm{T}$, we write $[\mathrm{S}, \mathrm{T}]$ for the set of all situations that follow $\mathrm{S}$ and precede $\mathrm{T}$ (this includes $\mathrm{S}$ and $\mathrm{T}$ ), and we call $[\mathrm{S}, \mathrm{T}]$ the closed interval between $\mathrm{S}$ and $\mathrm{T}$.

The set of all paths is the event tree's sample space, which we designate by $\Omega$. A subset of the sample space is a Moivrean event, and a function on the sample space is a variable.

In Section 1, following the custom in probability theory, we used "event," without qualification, to mean what we now call a Moivrean event, in honor of Abraham De Moivre. The adjective "Moivrean" is introduced here because we will need to distinguish the Moivrean concept from other event concepts, including a concept we encountered a moment ago- the concept of an instantaneous event represented by a situation.

3.3. Divergence. Two situations are divergent if they have no common successor. By Axiom T1, this is equivalent to their not being sequenced. Divergent situations are instantaneous events that cannot both happen. We write $\mathrm{S}$ divergent $\mathrm{T}$ when $\mathrm{S}$ and $\mathrm{T}$ are divergent.

We say that $\mathrm{S}$ implies $\mathrm{T}$ and write $\mathrm{S}$ implies $\mathrm{T}$ when every successor of $\mathrm{S}$ (including $\mathrm{S}$ itself) is sequenced with $\mathrm{T}$. This means that $\mathrm{S}$ cannot happen unless $\mathrm{T}$ happens (sooner, later, or at the same time).

We say that $\mathrm{S}$ foretells $\mathrm{T}$ and write $\mathrm{S}$ foretells $\mathrm{T}$ when $\mathrm{S}$ both precedes and implies $\mathrm{T}$. This is equivalent to the requirement that any path through $\mathrm{S}$ must later (or at the same 
time) go through $\mathrm{T}$ (see Figure 17). It means that $\mathrm{T}$ cannot happen strictly earlier than $\mathrm{S}$ but is inevitable (or happens at the same time) when $\mathrm{S}$ happens.

Like precedes, the binary relation foretells is a partial ordering on the event tree. The binary relation implies is transitive and reflexive, but not asymmetric and hence not a partial ordering. The binary relation divergent is symmetric.

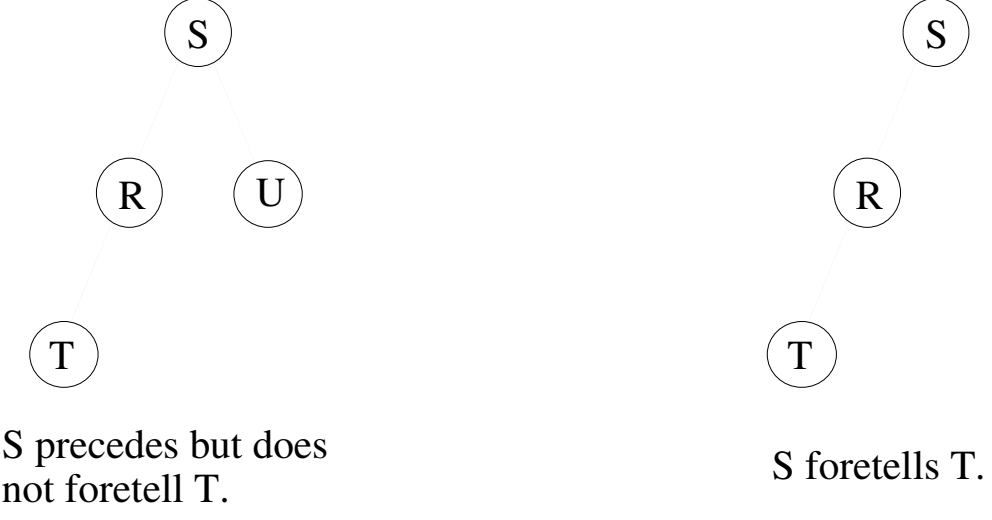

Figure 17 A situation $\mathrm{S}$ foretells a situation $\mathrm{T}$ if any path down the tree that passes through S must later pass through $\mathrm{T}$.

3.4. Clades. Let us call a nonempty set of divergent situations a clade. The interpretation of individual situations as instantaneous events extends to clades: a clade $\Xi$ is the instantaneous event that happens when and only when the event-tree observer arrives in a situation in $\Xi$. A clade, like a situation, can happen only once as the observer moves through the tree. The various relations we have defined for situations also extend to clades. Some of them (notably precedes and foretells) unpack in informative ways as they extend.

If $S$ is a situation, then $S$ and $\{S\}$, the clade consisting of $S$ alone, have the same meaning when they are considered as instantaneous events. We call $\{\mathrm{I}\}$, where I is the initial situation, the initial clade. 
When a path and a clade have a nonempty intersection, we say that the clade cuts the path, and that the path goes through the clade.

When two clades $\Xi$ and $\Phi$ are disjoint as sets, we write $\Xi$ separate $\Phi$. This means the two clades cannot happen at the same time.

We say $\Xi$ and $\Phi$ are divergent and write $\Xi$ divergent $\Phi$ if each situation in $\Xi$ is divergent from each situation in $\Phi$. This is equivalent to requiring that $\Xi$ and $\Phi$ be disjoint and their union be a clade. It means the two clades cannot both happen. It generalizes divergence for situations: $\{\mathrm{S}\}$ divergent $\{\mathrm{T}\}$ if and only if $\mathrm{S}$ divergent $\mathrm{T}$. If $\left\{\Xi_{\alpha}\right\}_{\alpha \in A}$ are divergent clades (if $\Xi_{\alpha}$ and $\Xi_{\beta}$ are divergent whenever $\alpha \neq \beta$ ), then the union $\cup_{\alpha \in \mathrm{A}} \Xi_{\alpha}$ is a clade.

Here are six more binary relations for clades:

- $\Xi$ prerequisite $\Phi$ ( $\Xi$ is a prerequisite for $\Phi)$ if every situation in $\Phi$ has a predecessor in $\Xi$. This means that $\Xi$ must happen before $\Phi$ can happen (though they may happen at the same time).

- $\Xi$ allows $\Phi(\Xi$ allows $\Phi)$ if every situation in $\Xi$ has a successor in $\Phi$. This means that $\Phi$ is possible (but cannot already have happened strictly earlier) whenever $\Xi$ happens. It does not rule out $\Phi$ happening and $\Xi$ not happening.

- $\Xi$ implies $\Phi$ ( $\Xi$ implies $\Phi)$ if any path through $\Xi$ also goes through $\Phi$. This means that $\Xi$ cannot happen unless $\Phi$ happens (sooner, later, or at the same time).

- $\Xi$ precedes $\Phi(\Xi$ precedes $\Phi)$ if $\Xi$ prerequisite $\Phi$ and $\Xi$ allows $\Phi$. This means that $\Phi$ cannot happen unless $\Xi$ happens first or at the same time, and $\Phi$ is possible whenever $\Xi$ happens.

- $\Xi$ foretells $\Phi$ ( $\Xi$ foretells or is a cause of $\Phi$ ) if $\Xi$ allows $\Phi$ and $\Xi$ implies $\Phi$. In other words, if a path goes through $\Xi$, it goes through $\Phi$ at the same time or later. This 
means that $\Phi$ cannot happen strictly earlier than $\Xi$ but is inevitable (or happens at the same time) whenever $\Xi$ happens. It does not rule out $\Phi$ happening and $\Xi$ not happening.

- $\Xi$ alw.foretells $\Phi$ ( $\Xi$ always foretells $\Phi)$ if $\Xi$ precedes $\Phi$ and $\Xi$ foretells $\Phi$. In other words, a path goes through $\Xi$ if and only if it goes through $\Phi$, and if it does go through them, it goes through $\Xi$ first or at the same time. This means that $\Phi$ cannot happen unless $\Xi$ happens first (or at the same time) and is inevitable (or happens at the same time) whenever $\Xi$ happens.

The relation implies is symmetric and transitive. The other five relations are partial orderings. The dependencies among the six relations are clarified by the Venn diagram in Figure 18. As this diagram indicates, $\Xi$ alw.foretells $\Phi$ can be defined from the other five relations in several ways. Instead of saying that it means $\Xi$ precedes $\Phi$ and $\Xi$ foretells $\Phi$, we can say, for example, that it means $\Xi$ prerequisite $\Phi$ and $\Xi$ implies $\Phi$.

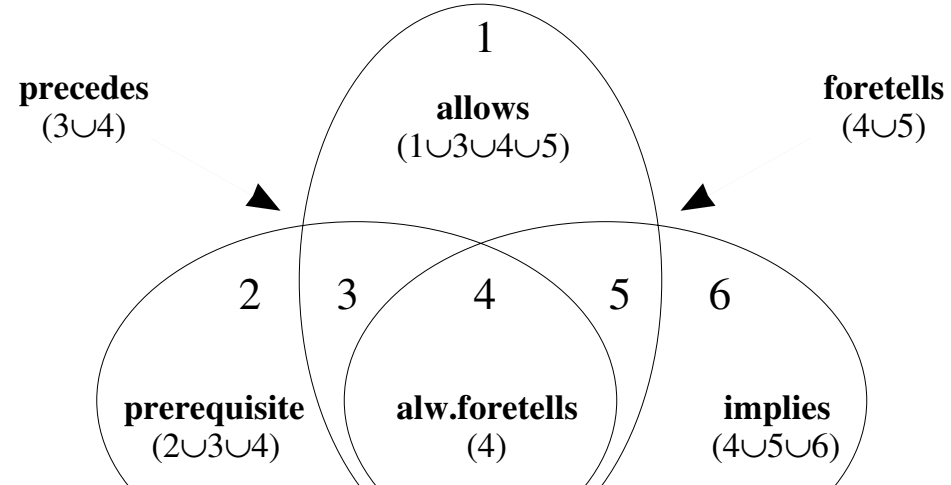

Figure 18 A Venn diagram for six binary relations on clades, thought of as sets of ordered pairs of clades. A Venn diagram for three sets can have as many as seven regions (not counting the region not in any of the sets), but the Venn diagram for 
prerequisite, allows, and implies has only six, because $\Xi$ prerequisite $\Phi$ and $\Xi$ implies $\Phi$ together imply $\Xi$ allows $\Phi$. By definition, precedes $=$ prerequisite $\cap$ allows and foretells $=$ allows $\cap$ implies. We can obtain alw.foretells in several ways; it is the intersection of either of the pair $\{$ prerequisite,precedes $\}$ with either of the pair $\{$ implies,foretells $\}$.

When the clades $\Xi$ and $\Phi$ are singletons, say $\Xi=\{S\}$ and $\Phi=\{T\}$, the six binary relations reduce to three. The two relations $\{\mathrm{S}\}$ prerequisite $\{\mathrm{T}\}$ and $\{\mathrm{S}\}$ allows $\{\mathrm{T}\}$ are equivalent to each other and hence to their conjunction, $\{\mathrm{S}\}$ precedes $\{\mathrm{T}\}$. And $\{S\}$ foretells $\{T\}$ and $\{S\}$ alw.foretells $\{T\}$ are equivalent. (See Figure 19.)

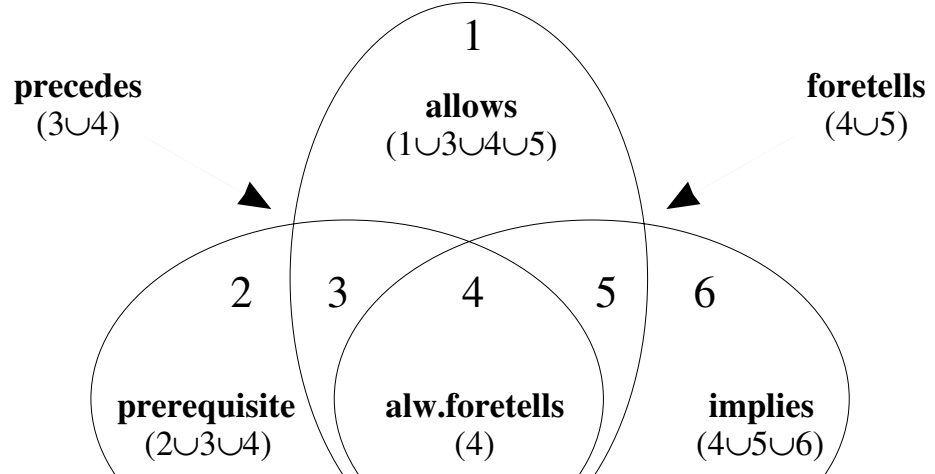

Figure 19 The six binary relations in Figure 16 reduce to three when we consider only individual situations. In this case, prerequisite is equal to allows and hence to their intersection, precedes. And alw.foretells and foretells are also equal.

We have defined precedes, foretells, and implies separately for clades and situations, but the definitions agree: $\{\mathrm{S}\}$ precedes $\{\mathrm{T}\}$ if and only if $\mathrm{S}$ precedes $\mathrm{T}$, and so on. Hence we can ignore the distinction between $\{\mathrm{S}\}$ and $\mathrm{S}$ when talking about relations between clades. The following equivalences are useful: 
- $\Xi$ prerequisite $\Phi$ if and only if $\Xi$ prerequisite T for every T in $\Phi$.

- $\Xi$ allows $\Phi$ if and only if S allows $\Phi$ for every $S$ in $\Xi$.

- $\Xi$ implies $\Phi$ if and only if $S$ implies $\Phi$ for every $S$ in $\Xi$.

- $\Xi$ foretells $\Phi$ if and only if S foretells $\Phi$ for every S in $\Xi$.

(The relations precedes and alw.foretells do not reduce in this way.) Of course, divergence and disjointness reduce completely to relations between individual situations:

- $\quad \Xi$ divergent $\Phi$ if and only if $S$ divergent $\mathrm{T}$ for every $\mathrm{S}$ in $\Xi$ and $\mathrm{T}$ in $\Phi$.

- $\quad \Xi$ separate $\Phi$ if and only if $S \neq \mathrm{T}$ for every $\mathrm{S}$ in $\Xi$ and $\mathrm{T}$ in $\Phi$.

3.5. Cuts. When a clade $\Phi$ consists of successors of a situation $S$, and every path through $\mathrm{S}$ also goes through $\Phi$, we say that $\Phi$ is a cut of $S .{ }^{4}$ Intuitively, a cut of $\mathrm{S}$ is an instantaneous event that cannot happen strictly before $\mathrm{S}$ happens or without $\mathrm{S}$ happening but must eventually happen once $S$ has happened. The clade $\{S\}$ qualifies as a cut of $S$.

The condition S alw.foretells $\Phi$ is equivalent to $\Phi$ being a cut of S. The condition $\Xi$ foretells $\Phi$ is equivalent to every situation in $\Xi$ having a cut in $\Phi$. The condition $\Xi$ implies $\Phi$ is equivalent to every situation in $\Xi$ having either a predecessor or a cut in $\Phi$. We refer to a cut of the initial situation simply as a cut. A cut is simply a clade that cuts every path. Intuitively, it is an instantaneous event that must eventually happen (see Figure 20).

${ }^{4}$ This differs from the definition of cut given in Section 11.2 of The Art of Causal Conjecture. 


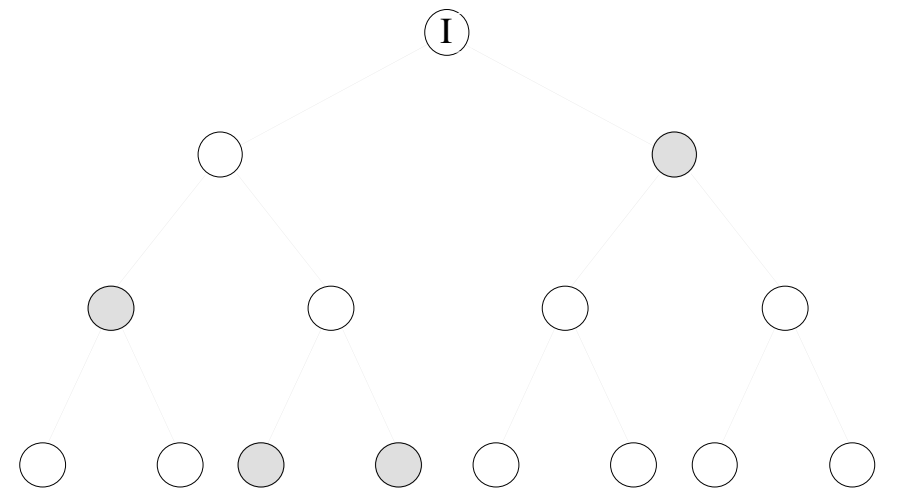

Figure 20 A cut.

Any cut is a maximal clade - a clade that is not contained in a larger clade. In a finite event tree, the converse is also true: any maximal clade is a cut. But as Figure 21 illustrates, a maximal clade may fail to be a cut in an infinite event tree. A clade cannot always be enlarged to a cut.

I

$\mathrm{S}_{1}$

$\mathrm{S}_{2}$

$\mathrm{S}_{3}$

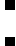

-

Figure 21 An event tree with one infinitely long path. This path does not go through the clade $\left\{S_{1}, S_{2}, \ldots\right\}$, which therefore is not a cut, although it is a maximal set of divergent situations.

The following proposition tells us more about cuts. 


\section{Proposition 5.}

1. If $T$ is a successor of $S$, then there is at least one cut of $S$ that contains $T$.

2. A set of sequenced successors of $S$ is a path from $S$ if and only if it intersects every cut of $S$.

3. Suppose $S$ is a situation, $\Phi$ is a set of divergent successors of $S$, and $\omega_{0}$ is a set of sequenced successors of $S$. Suppose (1) no situation in $\Phi$ precedes a situation in $\omega_{0}$, and (2) for every situation in $\Phi$, there is a divergent situation in $\omega_{0}$. Then $\omega_{0}$ can be enlarged to a path from $S$ that does not go through $\Phi$. (An example is provided by Figure 21, with $\Phi$ equal to the clade $\left\{S_{1}, S_{2}, \ldots\right\}$ and $\omega_{0}$ an infinite subset of the infinitely long path.)

Proof 1. If $\omega$ is a path from $S$ that does not go through $\mathrm{T}$, then we can choose a situation $\mathrm{U}$ in $\omega$ that is divergent from $\mathrm{T}$, thus obtaining a clade $\{\mathrm{T}, \mathrm{U}\}$ of successors of $\mathrm{S}$ that contains T and cuts $\omega$. More generally, if $\Xi$ is a clade of successors of $S$ that includes $T$, and $\omega$ is a path from $S$ not cut by $\Xi$, then we can construct another clade of successors of S, say $\Xi^{\prime}$, that contains T and cuts both $\omega$ and all the paths from S already cut by $\Xi$. (Choose a situation $\mathrm{U}$ divergent from $\mathrm{T}$ that cuts $\omega$. If $\mathrm{U}$ is divergent from all the situations in $\Xi$, then form $\Xi^{\prime}$ by adding $U$ to $\Xi$. If $U$ is sequenced with one or more situations in $\Xi$, then it must be their strict predecessor, and we can form $\Xi$ ' by adding $U$ and removing these strict successors of U.) It follows by the axiom of choice that there exists a clade of successors of $\mathrm{S}$ that contains $\mathrm{T}$ and cuts all paths from $\mathrm{S}$.

2. The definition of cut tells us that a path from $\mathrm{S}$ intersects every cut of S. Suppose, conversely, that $\omega$ is a set of sequenced successors of $S$ that intersects every cut of $S$. Consider any successor T of S not in $\omega$. By Statement 1 , there is a cut $\Xi$ of $S$ that contains $\mathrm{T}$, and by hypothesis, $\omega$ will contain a situation $\mathrm{U}$ that is also in $\Xi$. Since $\mathrm{T}$ and $\mathrm{U}$ are both in $\Xi$, they are divergent, and hence $\mathrm{T}$ is not sequenced with all the situations in 
$\omega$. This shows that $\omega$ is a maximal set of sequenced successors of $\mathrm{S}-$-i.e., that it is a path from $S$.

3. We begin by enlarging $\omega_{0}$ by adding all predecessors of its situations; the resulting set $\omega_{1}$ will still be sequenced and satisfy conditions (1) and (2). Any situation sequenced with all the situations in $\omega_{1}$ will follow all of them and hence will be divergent from $\Phi$. Hence it can be added to $\omega_{1}$ while preserving conditions (1) and (2). It follows by the axiom of choice that $\omega_{0}$ can be enlarged to a path without adding any situation in $\Phi$.

3.6. Subordinate Event Trees. Suppose $\Gamma$ is an event tree, with precedence ordering precedes, and suppose $\Gamma_{0}$ is a subset of $\Gamma$ satisfying this axiom:

Axiom ST0 $\Gamma_{0}$ contains a situation, say $\mathrm{I}_{0}$, that precedes all its other situations.

Then $\Gamma_{0}$ will qualify mathematically an event tree with the inherited precedence ordering (S precedes $\mathrm{T}$ in $\Gamma_{0}$ if and only if $\mathrm{S}$ and $\mathrm{T}$ are in $\Gamma_{0}$ and $\mathrm{S}$ precedes $\mathrm{T}$ in $\Gamma$ ). Axiom ST0 assures that $\Gamma_{0}$ has an initial situation (not necessarily the same as $\Gamma$ 's) and thus satisfies Axiom T0, and precedes satisfies Axiom $\mathrm{T} 1$ in $\Gamma_{0}$ because it does so in $\Gamma$. So we may call $\Gamma_{0}$ a subtree of $\Gamma$.

We need more than Axiom ST0, however, if we want to preserve relations of necessity when we move between $\Gamma$ and $\Gamma_{0}$. Figure 17 illustrates the issue. The smaller tree in that figure is a subtree of the larger tree, but as an event tree, it asserts that $\mathrm{S}$ foretells $\mathrm{T}$, which is not true in the larger tree. This suggests that we require the following axiom:

Axiom ST1 If $\Xi$ is a cut of $\mathrm{I}_{0}$ in $\Gamma_{0}$, then $\Xi$ is a cut of $\mathrm{I}_{0}$ in $\Gamma$. 
When a subtree $\Gamma_{0}$ satisfies Axiom ST1 as well as Axiom ST0, we say it is subordinate to $\Gamma$. (More colloquially, it is a sub event tree.) The subtree in Figure 15 does not satisfy ST1, because $\{\mathrm{T}\}$ is a cut of $\mathrm{S}$ in the smaller tree but not in the larger tree.

The following proposition clarifies the meaning of Axiom ST1.

Proposition 6. Suppose $\Gamma$ is an event tree. And suppose the subset $\Gamma_{0}$ of $\Gamma$ satisfies Axiom STO and hence is an event tree with the inherited precedence ordering, with $I_{0}$ as its initial situation. Then the following conditions are equivalent.

1. (Axiom ST1) If $\Xi$ is a cut of $I_{0}$ in $\Gamma_{0}$, then $\Xi$ is a cut of $I_{0}$ in $\Gamma$.

2. If $\omega$ is a path in $\Gamma$, and $\omega \cap \Gamma_{0}$ is nonempty, then $\omega \cap \Gamma_{0}$ is a path in $\Gamma_{0}$.

3. A nonempty set of situations in $\Gamma_{0}$ is a path in $\Gamma_{0}$ if and only if it is the intersection with $\Gamma_{0}$ of a path in $\Gamma$.

4. A set of situations in $\Gamma_{0}$ is a cut of $I_{0}$ in $\Gamma_{0}$ if and only if it is a cut of $I_{0}$ in $\Gamma$.

Proof 1. To show that Statement 1 implies Statement 2, consider a path $\omega$ in $\Gamma$, such that $\omega \cap \Gamma_{0}$ is nonempty. Since $\omega \cap \Gamma_{0}$ is nonempty, goes through $\mathrm{I}_{0}$, and hence it goes through every cut of $\mathrm{I}_{0}$ in $\Gamma$. It follows by Statement 1 that it goes through every cut of $\mathrm{I}_{0}$ in $\Gamma_{0}$, and hence that it is a path in $\Gamma_{0}$.

2. To show that Statement 2 implies Statement 1 , consider a cut of $I_{0}$ in $\Gamma_{0}$. It will cut every path in $\Gamma_{0}$, and by Statement 2 , this means that it will cut every path in $\Gamma$ that goes through $\mathrm{I}_{0}$ and so has a nonempty intersection with $\Gamma_{0}$. Thus it is a cut of $\mathrm{I}_{0}$ in $\Gamma$.

3. Statement 3 obviously implies Statement 2. To show that the two are equivalent, we must use Statement 2 to show that any path in $\Gamma_{0}$ is the intersection with $\Gamma_{0}$ of a path in $\Gamma$. Let $\omega_{0}$ be a path in $\Gamma_{0}$. Since $\omega_{0}$ is a set of sequenced situations in $\Gamma_{0}$, the axiom of 
choice assures that it can be enlarged to a path, say $\omega$, in $\Gamma$. By Statement $2, \omega \cap \Gamma_{0}$ is a path in $\Gamma_{0}$. Since it contains $\omega_{0}$, it is equal to $\omega_{0}$.

4. Statement 4 obviously implies Statement 1 . To complete the proof, notice that Statement 3 implies that if the clade $\Xi$ in $\Gamma_{0}$ is a cut of $I_{0}$ in $\Gamma$, then $\Xi$ is a cut of $I_{0}$ in $\Gamma_{0}$. As a cut of $I_{0}$ in $\Gamma, \Xi$ cuts every path in $\Gamma$ that goes through $I_{0}$. So by Statement $3, \Xi$ cuts all paths in $\Gamma_{0}$ and hence is a cut of $\mathrm{I}_{0}$ in $\Gamma_{0}$.

A subordinate event tree preserves all eight of the binary relations we have been studying.

Proposition 7. Suppose $\Gamma_{0}$ is an event tree subordinate to $\Gamma$. Suppose $\Xi$ and $\Phi$ are clades in $\Gamma_{0}$. Then $\Xi$ separate $\Phi$ in $\Gamma_{0}$ if and only if $\Xi$ separate $\Phi$ in $\Gamma$. And the analogous statements hold for divergent, prerequisite, allows, implies, precedes, foretells, and alw.foretells. Proof The disjointness of two sets is not affected, of course, by what larger set we place them in. The relations divergent, prerequisite, and allows are preserved because they depend only on relations of precedence between situations in the clades involved, and these relations are inherited by $\Gamma_{0}$ from $\Gamma$. By Statement 3 of Proposition 6 , implies is preserved. (Since $\Xi$ and $\Phi$ are clades in $\Gamma_{0}$, the condition that a path in $\Gamma$ through $\Xi$ also go through $\Phi$ is equivalent to the same condition on the path's intersection with $\Gamma_{0}$.) Finally, since precedes, foretells, and alw.foretells are defined in terms of prerequisite, allows, and implies, they are also preserved.

3.7. Humean Events. Thought of as an event, a situation $S$ or a clade $\Xi$ is instantaneous; it is the arrival of the event-tree observer in S or $\Xi$. In discussions of causality, we may also be interested in events that are not instantaneous. Such events can 
be thought of as changes from one clade to another, and hence can be represented as pairs of clades.

Formally, if $\Xi$ precedes $\Phi$, then we call the pair $(\Xi, \Phi)$ a Humean event. The clade $\Xi$ is its tail, and the clade $\Phi$ is its head. The head and tail may be identical; in this case the Humean event is instantaneous. A Humean event is simple if its head and tail both consist of single situations, initial if its tail is initial, inevitable if its head is a cut (in which case its tail is also a cut), full if its tail implies its head (since the tail precedes the head, this is the same as requiring that the tail cause the head, or that the tail be a full cause of the head) and proper if the head does not include a cut of any of the situations the tail. Two Humean events are divergent if their heads are divergent.

A simple Humean event $(\mathrm{S}, \mathrm{T})$ can be identified with the closed interval $[\mathrm{S}, \mathrm{T}]$. More generally, any Humean event $(\Xi, \Phi)$ can be thought of as the set consisting of all closed intervals that begin in $\Xi$ and end in $\Phi$.

A step from a situation $\mathrm{S}$ to a daughter $\mathrm{T}$ in a finite event tree is, of course, a closed interval and hence a Humean event. But individual steps cannot play in a general theory the special role they play when the event tree is finite. A daughter of $\mathrm{S}$ is a nearest strict successor-a strict successor of $\mathrm{S}$ that has no strict predecessor that is also a strict successor of S. In infinite event trees, where situations can have strict successors without having nearest strict successors, we must look to Humean events to play the philosophical and mathematical roles assigned to steps in Section 1: Humean events can represent contingent causes, and as we will see in the next section, we can apportion a variable's variance among Humean events.

Like clades, Humean events retain their identity, in a certain sense, under refinement. If $\rho$ refines $\Gamma$ to $\Gamma^{*}$, then $(\Xi, \Phi)$ and $(\rho(\Xi), \rho(\Phi))$ represent the same Humean event. 


\section{Martingales in Event Trees}

Turning an event tree into a probability tree is simple in the finite case: we merely add branching probabilities below each nonterminal situation. This approach does not always work for infinite trees, for two reasons. One the one hand, there may be a continuum of daughters. On the other hand there may be no daughters at all, as in the example at the end of Subsection 3.1. How can we proceed?

The obvious answer is to shift attention from branching probabilities to expected values. Expected values are assigned to situations, not to branches or steps, and situations remain prominently present in our general theory of event trees. Instead of thinking of a probability structure as a collection of branching probabilities, we think of it as a linear space of functions-martingales, we shall call them-that assign numbers (intuitively, expected values for numerical variables) to situations. Instead of axiomatizing probabilities we axiomatize martingales.

This approach has a number of advantages. Most importantly, it points the way to generalizations that allow probability to play a wider role in causal reasoning. Traditionally, probability theory has insisted that probabilities be defined for all events, and in the context of a finite event tree, this means that every step should have a branching probability. But when we think of probability structure in terms of a linear space of martingales, it is no longer natural to insist that the structure be complete in this way. Each martingale in the structure is a declaration by the event-tree observer that certain bets are fair-that they are among the bets the observer can make and expect to break even —and the observer may have this kind of confidence in only a limited number of bets. The events represented in the tree may include the observer's own action, on which it may not be sensible for her to bet. And when the event tree and the probability structure represent nature, so that the martingales represent regularities that nature can 
use for prediction, the extent of such regularities should be regarded as an empirical question.

4.1. Martingales Defined. Let us call a numerical function on an event tree $\Gamma$ a process. A process $\mu$ is a martingale if it satisfies the following axiom:

Axiom $\mathbf{M}$ If $S \in \Gamma$, and $\Xi$ is a cut of $S$, then there exist situations $T_{1}$ and $T_{2}$ in $\Xi$ such that $\mu\left(\mathrm{T}_{1}\right) \leq \mu(\mathrm{S}) \leq \mu\left(\mathrm{T}_{2}\right)$.

If the event tree is finite, this is equivalent to saying that every nonterminal situation $\mathrm{S}$ has daughters $T_{1}$ and $T_{2}$ such that $\mu\left(T_{1}\right) \leq \mu(S) \leq \mu\left(T_{2}\right)$.

For every number a, the process that is identically equal to a is a martingale. If $\mu$ is a martingale, and a is a number, then a $\mu$ is a martingale. More generally, if $\mu$ is a martingale, and $\mathrm{f}$ is a monotonic function, then $\mathrm{f}(\mu)$ is a martingale. On the other hand, as Figure 22 illustrates, the pointwise sum of two martingales is not necessarily a martingale.

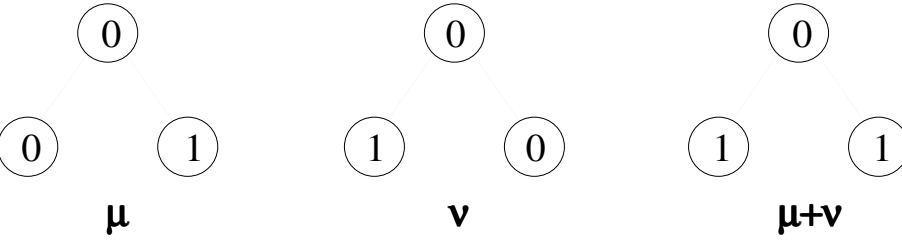

Figure 22 The processes $\mu$ and $v$ are martingales, but the process $\mu+v$ is not.

The expected values of a numerical variable in a finite probability tree obviously form a martingale. More precisely, if $\mathrm{X}$ is a variable in a finite probability tree, and we define a process $\mathbf{E}(\mathrm{X})$ on the tree by setting 
(7) $\mathrm{E}(\mathrm{X})(\mathrm{S}):=\mathrm{E}_{\mathrm{S}}(\mathrm{X})$,

then $\mathrm{E}(\mathrm{X})$ is a martingale. And the variable $\mathrm{X}$ is identified by $\mathrm{E}(\mathrm{X})$; its value on a path is given by $\mathbf{E}(\mathrm{X})$ 's value on the terminal situation in that path. The abstract concept of martingale does not depend, however, on the event tree having been made into a probability tree by the addition of branching probabilities. Nor does a martingale necessarily determine a variable.

We say a process is determinate in a situation $\mathrm{S}$ if all $\mathrm{T}$ that follow $\mathrm{S}$ are assigned the same value as $\mathrm{S}$ by the process. We say a process is terminating if it is determinate in some cut. If a process is terminating, then it determines a variable, whose value on each path is the value the process eventually assumes on that path. We then say that the process evaluates the variable, and we may think of the values of the process as "expected values" of the variable. But we need not assume that a martingale always settles down in this way.

4.2. Catalogs. We call a linear space of martingales on an event tree a catalog. Catalogs provide the principal concept of probability structure in the remainder of this article. Intuitively, a catalog represents all the gambling strategies an observer in the event tree considers fair. The assumption that these strategies form a linear space derives from the judgment that fairness is preserved under the formation of linear combinations. If a given bet is fair, then it is fair to double it, and if two bets are individually fair, then it is fair to combine them.

A catalog can contain at most one martingale evaluating a given variable. (If two martingales in a catalog evaluate the same variable, then their difference, which is also in the catalog and is therefore a martingale, will have the value zero everywhere by Axiom M.) Whenever the catalog does contain a martingale that evaluates $\mathrm{X}$, we write $\mathrm{E}(\mathrm{X})$ for 
this martingale, and we call $\mathrm{E}(\mathrm{X})$ the expectation of $\mathrm{X}$. We write $\mathrm{E}_{\mathrm{S}}(\mathrm{X})$ for $\mathrm{E}(\mathrm{X})(\mathrm{S})$, and we call $\mathrm{E}_{\mathrm{S}}(\mathrm{X})$ the expected value of $\mathrm{X}$ in $\mathrm{S}$.

In the case of a variable $\mathrm{E}$ that takes only the values zero and one (an event), we write $\mathbf{P}(E)$ instead of $\mathbf{E}(E)$ and $P_{S}(E)$ instead of $E_{S}(E)$; we call the martingale $\mathbf{P}(E)$ the probability of $\mathrm{E}$, and we call the number $\mathrm{P}_{\mathrm{S}}(\mathrm{E})$ the probability of $\mathrm{E}$ in $\mathrm{S}$. It often happens that a catalog evaluates a variable $X$ even though it does not evaluate all events of the form $\{X=x\}$. When a catalog evaluates all events of the form $\{X=x\}$, we say it probabilizes X.

In the case of a finite probability tree, the set of martingales $\{E(X) \mid X$ is a variable $\}$, where $\mathbf{E}(\mathrm{X})$ is given by (7), is a catalog. Such a catalog is maximal; since it already contains a martingale evaluating every variable, it is not a subspace of a strictly larger catalog. In general, we call a maximal catalog on an event tree a probability catalog. While probability catalogs are interesting, smaller catalogs are also interesting, even in the case of finite event trees. An important example is the finite decision tree, where branching probabilities are provided for the branchings below chance situations, but not for those below decision situations. Figure 23 gives an example. If we form martingales by placing bets according to the odds given by the branching probabilities below chance situations, then these martingales form a catalog, which we call the fair-bet catalog for the decision tree. Each martingale in the catalog evaluates a variable, but not all variables are evaluated by martingales in the catalog; the evaluated variables satisfy linear constraints imposed by the fact that the martingales are all constant on the daughters of each decision situation. For details, and for an analysis of the structure of all catalogs on finite event trees, see Section 12.2 of The Art of Causal Conjecture. 


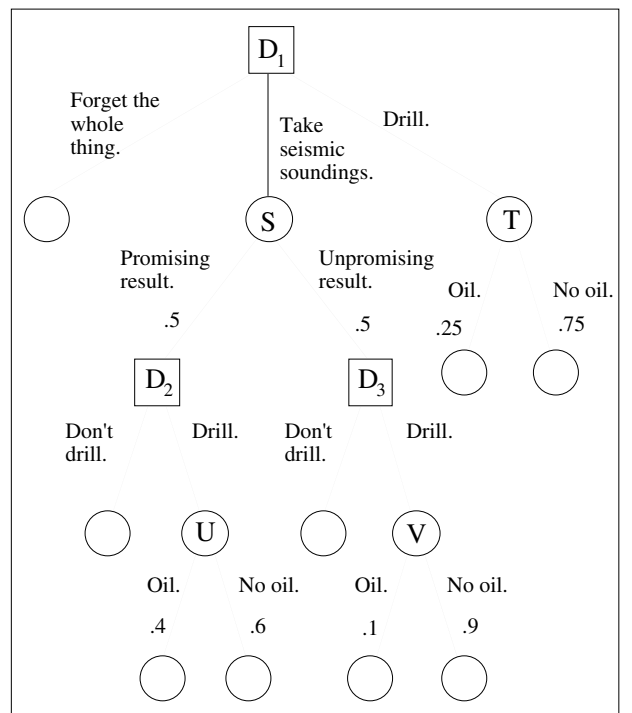

A Decision Tree

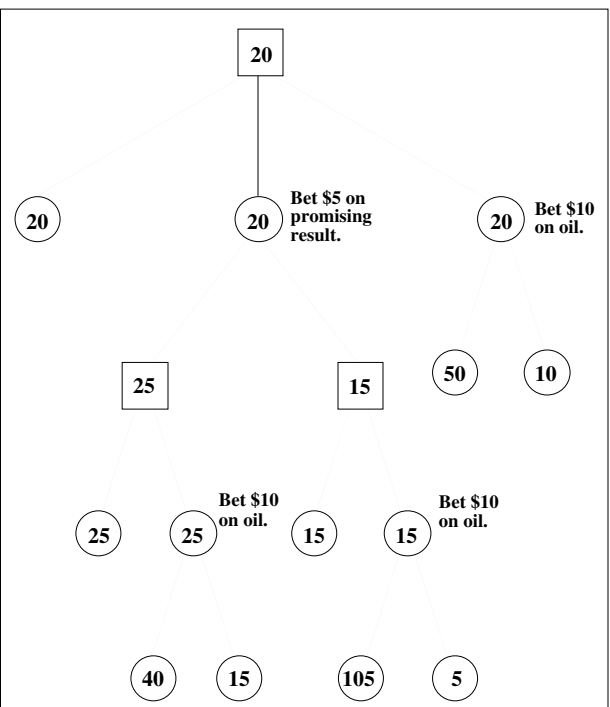

A Martingale for the Decision Tree

Figure 23 The decision tree on the left is adapted from the problem of the oil wildcatter in Raiffa (1968). There are branching probabilities for the steps following the nonterminal chance situations $\mathrm{S}, \mathrm{T}, \mathrm{U}$, and $\mathrm{V}$, but no branching probabilities following the decision situations $\mathrm{D}_{1}, \mathrm{D}_{2}$, and $\mathrm{D}_{3}$. On the right is a martingale obtained by betting according to the branching probabilities that are given.

In principle, any catalog can be enlarged to a probability catalog, and we might take the view that a catalog that falls short of being a probability catalog is an incomplete expression of opinion. This seems wrong-headed, however. If we take a constructive view of probability judgment (Shafer and Tversky 1985), then we expect a person's probability opinions to be limited. And if we think of nature's probabilities as reflecting regularities in the world, then we have no reason to believe that such regularities are to be found in every direction. 
4.3. Stopping a Martingale. If $\mu$ is a martingale, and $\Xi$ is a cut, then we write $\mathbf{E}_{\Xi}(\mu)$ for the process given by

$$
\mathrm{E}_{\Xi}(\mu)(\mathrm{S}):=\mu(\Xi(\mathrm{S}))
$$

where

$$
\Xi(S):=\left\{\begin{array}{l}
S \text { if } T \in \Xi \text { and } S \text { precedes } T \\
R \text { if } R \in \Xi \text { and } R \text { precedes } S .
\end{array}\right.
$$

It is easy to see that $\mathbf{E}_{\Xi}(\mu)$ is a martingale. We call it the expectation of $\mu$ in $\Xi$.

Intuitively, it is the result of stopping $\mu$ in $\Xi$. Figure 24 illustrates the idea.

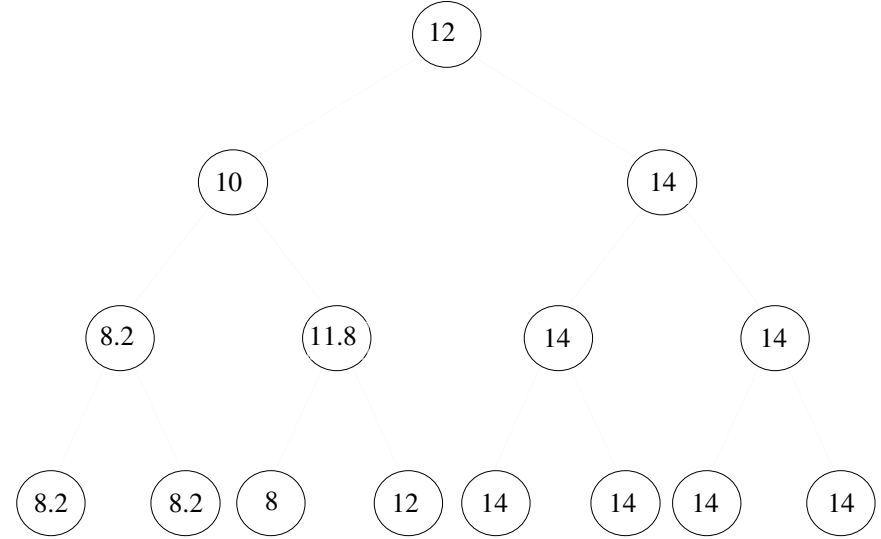

Figure 24 The martingale $\mathbf{E}_{\Xi}(\mu)$, where $\mu$ is the martingale in Figure 4, and $\Xi$ is the cut in Figure 20. This martingale agrees with $\mu$ down to $\Xi$ and then remains unchanged the rest of the way down the tree.

The expectation of $\mu$ given $\Xi$ is determinate in $\Xi$. If $\mu$ is determinate in $\Xi$, then $\mathbf{E}_{\Xi}(\mu)=\mu$. If $\Xi$ and $\Phi$ are cuts and $\Xi$ precedes $\Phi$, then

$$
\mathbf{E}_{\Xi}\left(\mathbf{E}_{\Phi}(\mu)\right)=\mathbf{E}_{\Xi}(\mu)
$$

an event-tree form of the rule of iterated expectation.

We call a catalog MDoob if it satisfies the following axiom: 
Axiom $\mathbf{V}$ If $\mu \in \mathrm{M}$ and $\Xi$ is a cut, then $\mathrm{E}_{\Xi}(\mu) \in \mathrm{M}$.

Probability catalogs are Doob, as are the fair-bet catalogs for decision trees. A catalog that is not Doob can always be enlarged to one that is by adding all finite linear combinations of its expectations and its elements. The idea of fairness could justify our incorporating Axiom $\mathrm{V}$ into the definition of catalog, but since any linear space of martingales can be enlarged in a canonical way to satisfy them, simplicity and convenience is best served by calling any linear space of martingales a catalog.

4.4. Lower and Upper Probabilities. Consider an event tree $\Gamma$ with a fixed Doob catalog M. We say that a process $\mu$ on $\Gamma$ is eventually less than or equal to a variable $\mathrm{X}$ after $S$, and we write $\mu \leq \downarrow_{S} X$, if in every path $\omega$ from $S$, there is a situation $T$ such that $\mu(\mathrm{U}) \leq \mathrm{X}(\omega)$ for all $\mathrm{U}$ following $\mathrm{T}$. We define $\mu \geq \downarrow_{\mathrm{S}} \mathrm{X}$ similarly.

Given a variable $X$, we set

$$
\mathrm{E}_{\mathrm{S}}^{-}(\mathrm{X}):=\sup \left\{\mu(\mathrm{S}) \mid \mu \in \mathrm{M}, \mu \leq \downarrow_{\mathrm{S}} \mathrm{X}\right\},
$$

and we write $\mathbf{E}^{-}(\mathrm{X})$ for the process given by $\mathbf{E}^{-}(\mathrm{X})(\mathrm{S}):=\mathrm{E}_{\mathrm{S}}^{-}(\mathrm{X})$. We call $\mathbf{E}^{-}(\mathrm{X})$ the lower expectation of $\mathrm{X}$, and we call $\left.\mathrm{E}_{\mathrm{S}}^{-} \mathrm{X}\right)$ the lower expected value of $\mathrm{X}$ in $\mathrm{S}$.

Dual to the idea of lower expectation is the idea of upper expectation. The upper expected value of $\mathrm{X}$ in $\mathrm{S}$ is

$$
\mathrm{E}_{S}^{+}(\mathrm{X}):=\inf \left\{\mu(\mathrm{S}) \mid \mu \in \mathrm{M}, \mu \geq \downarrow_{\mathrm{S}} \mathrm{X}\right\},
$$

and the upper expectation of $\mathrm{X}$ is the process $\mathbf{E}^{+}(\mathrm{X})$ given by $\mathbf{E}^{+}(\mathrm{X})(\mathrm{S}):=\mathrm{E}_{\mathrm{S}}^{+}(\mathrm{X})$. Notice that $\mathbf{E}^{-}(X)=-\mathbf{E}^{+}(-X)$.

The ideas of lower expectation and upper expectation are most easily understood in terms of a house, an infinitely rich observer in the event tree, who is willing to make a bet 
with another observer whenever that bet is in the catalog or is more favorable to herself than one in the catalog. The lower expected value $E_{S}^{-}(X)$ is the highest price the house would pay for $\mathrm{X}$ in $\mathrm{S}$, while the upper expected value $\mathrm{E}_{S}^{+}(\mathrm{X})$ is the lowest price she would charge for $\mathrm{X}$ in $\mathrm{S}$. If the house were the only betting partner available to us, we would think of $E_{S}^{-}(X)$ as the scrap value of $X$ in $S$, and of $E_{S}^{+}(X)$ as the cost of $X$ in $S$.

Here are some informative properties of lower expectation.

\section{Proposition 8.}

1. The supremum in (8) is attained. In other words, for each $S$ there is a martingale $\mu$ in $M$ such that $\mu \geq \downarrow_{S} X$ and $\mu(S)=E_{S}^{-}(X)$.

2. $E^{-}(X)$ is a martingale.

3. $E^{-}(X)$ is the infimum of the expectations of $X$ for the various probability catalogs that contain $M$. There is a probability catalog containing $M$ for which $\mathbf{E}^{-}(X)$ is the expectation of $X$.

4. If $\Xi$ is evaluated by a martingale in $M$, so that the expectation $E(X)$ exists, then $E^{-}(X)=E(X)$.

(See Section 12.6 of The Art of Causal Conjecture. We leave it to the reader to formulate the corresponding statements about upper expectation.) According to Statement 3, lower expected values can be thought of as lower bounds. Upper expected values can similarly be thought of as upper bounds. Different ways of completing the definition of the branching probabilities in an event tree with a catalog produce different expected values for $\mathrm{X}$ in $\mathrm{S}$, and $\mathrm{E}_{\mathrm{S}}^{-}(\mathrm{X})$ and $\mathrm{E}_{\mathrm{S}}^{+}(\mathrm{X})$ are tight lower and upper bounds, respectively, on these expected values. 
If we are in a position to enlarge the catalog to a probability catalog, by taking actions that fix the branching probabilities, then it is appropriate to view lower expected and upper expected values as bounds on expected values. Figure 25 illustrates the point. But when it is not within our power to transform the tree into a probability tree by enlarging its catalog, the idea that the lower and upper expectations are bounds should be treated gingerly. There is no reason to say that there is an unknown expectation between the lower expectation and upper expectation.

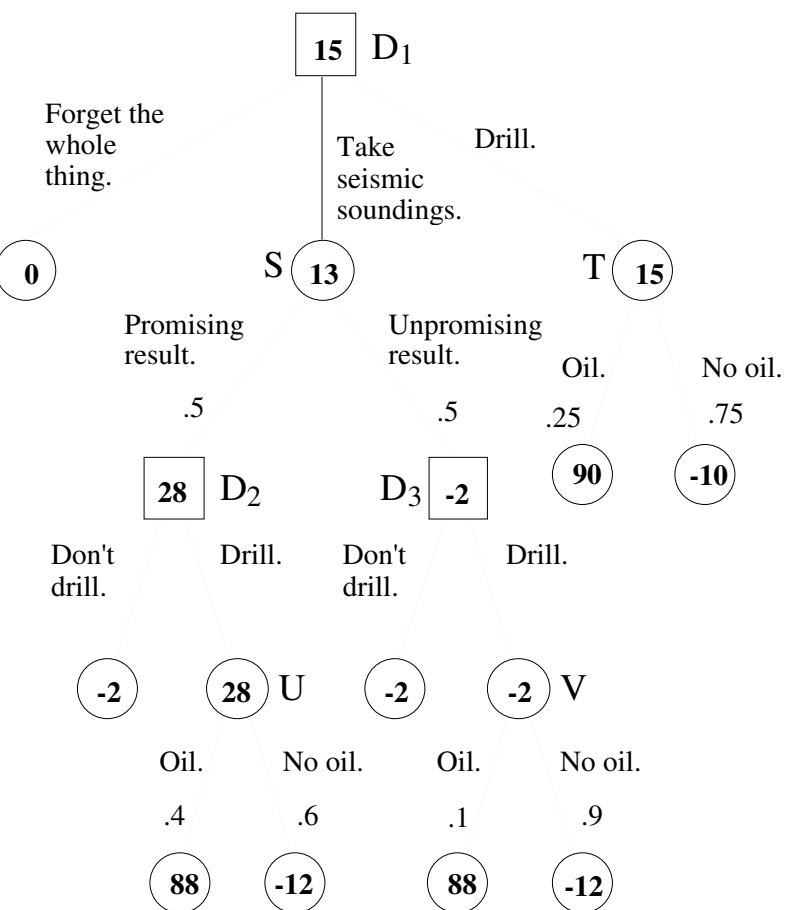

Figure 25 The upper expectation of a wildcatter's payoff. We assume that it costs $\$ 10,000$ to drill and $\$ 2,000$ to take seismic soundings, and that the wildcatter will earn $\$ 100,000$ if she finds oil. This gives the payoff $X$ shown in the terminal situations. The upper expected values of $\mathrm{X}$ are shown in the other situations. If the wildcatter wants to choose the branching probabilities in the decision situations so as to maximize $\mathrm{X}^{\prime} \mathrm{s}$ expected value in the initial situation, then the best she can do is $\mathbf{E}_{\Omega}^{+}(\mathrm{X})$, or $\$ 15,000$. 
This is achieved by drilling in the initial situation with probability one. The choice of branching probabilities for $\mathrm{D}_{2}$ and $\mathrm{D}_{3}$ is then immaterial, but in order to achieve the upper expected value in $\mathrm{D}_{2}$, we should drill with probability one there. Any choice of branching probabilities will achieve the upper expected value in $\mathrm{D}_{3}$.

When $\mathrm{E}$ is an event (a variable that takes only the values zero and one), we write $\mathrm{P}_{\mathrm{S}}^{-}(\mathrm{E})$ for $\mathrm{E}_{\mathrm{S}}^{-}(\mathrm{E})$ and call it the lower probability of $\mathrm{E}$ in $\mathrm{S}$. Similarly, we write $\mathrm{P}_{\mathrm{S}}^{+}(\mathrm{E})$ for $\mathrm{E}_{\mathrm{S}}^{+}(\mathrm{E})$ and call it the upper probability of $\mathrm{E}$ in $\mathrm{S}$. The lower probability $\mathrm{P}_{\mathrm{S}}^{-}(\mathrm{E})$ is the highest price the house would pay for a contract that returns $\$ 1$ if $E$ happens, while the upper probability $\mathrm{P}_{\mathrm{S}}^{+}(\mathrm{E})$ is the lowest price at which she would sell such a contract.

4.5. Causality. The theory of causality that we developed in Section 1 can be extended to the general context of event trees with catalogs provided that we make appropriate regularity assumptions. The simplest adequate assumption, perhaps, is that each path in the event tree is compact in the interval topology. This means that for any collection of closed intervals (see Subsection 3.2) that has $\omega$ as its union, there is a finite subcollection that also has $\omega$ as its union.

Let us call a Humean event of the form $(\mathrm{S}, \Xi)$, where $\Xi$ is a cut of $\mathrm{S}$, an experiment, and let us call the set of situations

$\{T$ I S precedes $T$ and there exists $U \in \Xi$ such that $T$ precedes $U$ \} the scope of the experiment $(\mathrm{S}, \Xi)$. Let us call a collection of experiments a cover if the union of the scopes is equal to the entire event tree.

We say that an experiment forbears a martingale if the martingale has the same value on all situations in the experiment's scope. We say that two martingales are uncorrelated if there exists a cover, every experiment in which forbears at least one of the two 
martingales. The following proposition is about uncorrelated martingales is illustrated by Figure 26.

Proposition 9. Suppose $\mu$ and $v$ are uncorrelated martingales. Then $\mu+v$ and $\mu v$ are martingales. More generally, $f(\mu, v)$ is a martingale whenever $f(x, y)$ is monotonic in both its arguments.

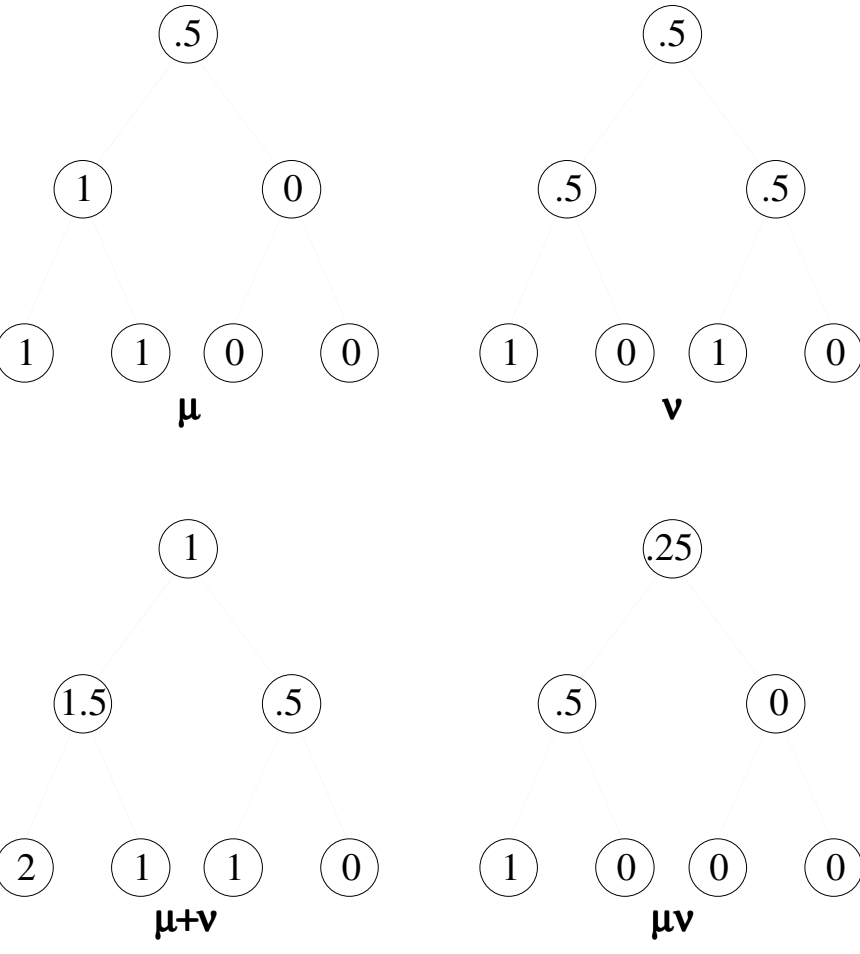

Figure 26 Two uncorrelated martingales, their sum, and their product.

We say that two variables $\mathrm{Y}$ and $\mathrm{Z}$ are causally uncorrelated if they are evaluated and the martingales evaluating them are uncorrelated. The next proposition then spells out within in this general theory the fact, which we took notice of for finite probability trees in Section 1, that causal uncorrelatedness implies uncorrelatedness in the usual sense. 
Proposition 10. Consider an event tree with a Doob catalog M.

1. If $\mu$ and $v$ are uncorrelated martingales in $M$, then $\mu v$ is in $M$.

2. If $X$ and $Y$ are uncorrelated variables, then the product $X Y$ is evaluated, and $E(X Y)$ $=E(X) E(Y)$.

From the idea of causal uncorrelatedness, we can also construct the other causal ideas that we mentioned in Section 1. For example, we say that two variables $\mathrm{X}$ and $\mathrm{Y}$ are causally independent if they are probabilized and $\{\mathrm{X}=\mathrm{x}\}$ is uncorrelated with $\{\mathrm{Y}=\mathrm{y}\}$ for all $\mathrm{x}$ and $\mathrm{y}$, and we can then prove that causally independent variables are independent in the usual sense.

4.6. Limit Theorems. The idea of lower probability is important because it allows us to formulate the traditional limit theorems of probability theory, which assert that various things are almost certain to happen in a long run of trials. We simply interpret "almost certain" as "with very high lower probability."

An example of this approach is provided by the following version of the law of large numbers.

Proposition 11. For any positive real number $K$ (no matter how large) and any positive real numbers $\varepsilon$ and $\delta$ (no matter how small), there exists an integer $N$ such that if $n \geq N$ and $X_{1}, X_{2}, . ., X_{n}$ are causally uncorrelated variables in an event tree with a Doob catalog, all the $X_{i}$ have value zero in the initial situation $I$, all are bounded by $K$, and $\bar{X}$ is their

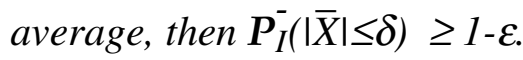


The law of large numbers has the same significance in our theory as it does in standard probability theory. It implies that if situations are replicated as nature moves through her tree, we can estimate nature's probabilities in these situations from observed frequencies.

Other limit theorems, especially the law of large numbers and the law of the iterated logarithm, and have been treated from essentially the point of view taken here by Vovk (1993a,b).

\section{Refinement of Abstract Event Trees}

How can we describe abstractly the relation between an event tree and a refinement? The examples in Section 2 suggest that we specify, for each situation $\mathrm{S}$ in the original tree, the situations in the refinement that agree with S, in the sense that they represent the same instantaneous event, possibly describing it in more detail. These situations are divergent-i.e., they form a clade, say $\rho(S)$ in the refinement. If we set $\rho(\Xi):=$ $\cup_{S \in \Xi} \rho(S)$ when $\Xi$ is a clade in the original tree, then $\rho(\Xi)$ is also a clade in the refinement, consisting of situations that agree with and possibly add more detail to some situation in $\Xi$. The mapping from clades to clades thus defined fully specifies the relationship between the two event trees.

5.1. An Axiomatization of Refinement. Formally, let us say that a mapping $\rho$ from clades in an event tree $\Gamma$ to clades in an event tree $\Gamma^{*}$ refines $\Gamma$ to $\Gamma^{*}$ if it satisfies the following axioms: 
Axiom R0 $\Xi$ is initial if and only if $\rho(\Xi)$ is initial. ${ }^{5}$

Axiom R1 $\Xi$ separate $\Phi$ if and only if $\rho(\Xi)$ separate $\rho(\Phi)$.

Axiom R2 $\Xi$ divergent $\Phi$ if and only if $\rho(\Xi)$ divergent $\rho(\Phi)$.

Axiom R3 $\Xi$ prerequisite $\Phi$ if and only if $\rho(\Xi)$ prerequisite $\rho(\Phi)$.

Axiom R4 $\Xi$ allows $\Phi$ if and only if $\rho(\Xi)$ allows $\rho(\Phi)$.

Axiom R5 $\Xi$ implies $\Phi$ if and only if $\rho(\Xi)$ implies $\rho(\Phi)$.

Axiom R6 If $\left\{\Xi_{\alpha}\right\}_{\alpha \in A}$ are divergent clades in $\Gamma$, then $\rho\left(\cup_{\alpha \in A} \Xi_{\alpha}\right)=\cup_{\alpha \in A} \rho\left(\Xi_{\alpha}\right)$.

When $\rho$ refines $\Gamma$ to $\Gamma^{*}$, we say $\Gamma^{*}$ is a refinement of $\Gamma$, and $\Gamma$ is a coarsening of $\Gamma^{*}$.

Axioms R0-R6 are easy to justify on the understanding that (1) a clade represents the instantaneous event that the observer arrives in a situation in the clade, and (2) $\Xi$ and $\rho(\Xi)$ represent the same instantaneous event. From this perspective, the axioms merely say that the meaning of various relations among instantaneous events (being divergent, one being a prerequisite for the other, etc.) is not affected by the choice of an event tree in which to represent the events.

We will abbreviate $\rho(\{S\})$ to $\rho(S)$. By Axiom R2, the clades $\rho(S)$ are all disjoint. When $S^{*}$ is an element of $\rho(S)$, we say $S^{*}$ refines or is refinement of $S$, and $S$ coarsens or is a coarsening of $\mathrm{S}^{*}$.

Given a subset $\gamma^{*}$ of $\Gamma^{*}$, we write $\rho^{-1}\left(\gamma^{*}\right)$ for the subset of $\Gamma$ consisting of all coarsenings of situations in $\gamma^{*}$.

The following proposition clarifies some aspects of refinement.

5In other words, $\rho(\{\mathrm{I}\})=\left\{\mathrm{I}^{*}\right\}$, where I and $\mathrm{I}^{*}$ are the initial situations in $\Gamma$ and $\Gamma^{*}$, respectively. 
Proposition 12. Suppose $\rho$ refines $\Gamma$ to $\Gamma^{*}$, and suppose $\Xi$ and $\Phi$ are clades in $\Gamma$.

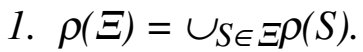

2. $\Xi=\Phi$ if and only if $\rho(\Xi)=\rho(\Phi)$.

3. $\Xi$ precedes $\Phi$ if and only if $\rho(\Xi)$ precedes $\rho(\Phi)$.

4. $\Xi$ foretells $\Phi$ if and only if $\rho(\Xi)$ foretells $\rho(\Phi)$.

5. $\Xi$ alw.foretells $\Phi$ if and only if $\rho(\Xi)$ alw.foretells $\rho(\Phi)$.

6. Suppose $S$ coarsens $S^{*}, T$ coarsens $T^{*}$, and $S^{*}$ precedes $T^{*}$. Then $S$ precedes $T$.

7. $\Phi$ contains a cut of $S$ if and only if $\rho(\Phi)$ contains a cut of every refinement of $S$.

8. Suppose $S^{*}$ and a successor $T^{*}$ refine situations in $\Gamma$. Then $T^{*}$ can be extended to a cut of $S^{*}$ consisting of situations that refine situations in $\Gamma$.

9. The event tree, say $\Gamma^{*} 0$, obtained by omitting from $\Gamma^{*}$ all situations that do not refine situations in $\Gamma$, is subordinate to $\Gamma^{*}$. Moreover, the mapping $\rho$, thought of as a mapping from clades of $\Gamma$ to clades of $\Gamma^{*} 0$, refines $\Gamma$ to $\Gamma^{*} 0$.

10. Suppose $T$ coarsens $T^{*}$ and $S$ precedes $T$. Then there exists a situation $S^{*}$ that refines $S$ and precedes $T^{*}$.

11. Suppose $S$ coarsens $S^{*}$ and $S$ precedes $T$. Then there exists a situation $T^{*}$ that follows $S^{*}$ and refines $T$.

12. Suppose $S$ coarsens $S^{*}$. Then (1) if $\omega^{*}$ is a path from $S^{*}$, then $\rho^{-1}\left(\omega^{*}\right)$ is a path from $S$, and (2) if $\omega$ is a path from $S$, then $\omega=\rho^{-1}\left(\omega^{*}\right)$ for some path $\omega^{*}$ from $S^{*}$.

13. Suppose $S$ coarsens $S^{*}$, and $\Phi$ is a cut of $S$. Then $\Phi=\rho^{-1}\left(\Phi^{*}\right)$ for some cut $\Phi^{*}$ of $S^{*}$.

Proof 1. Statement 1 is a special case of Axiom R6.

2. Since the $\rho(S)$ are disjoint, by Axiom R1, Statement 2 follows from Statement 1.

3. Statement 3 follows from Axioms R3 and R4.

4. Statement 4 follows from Axioms R4 and R5. 


\section{Statement 5 follows from Axioms R3 and R5.}

6. By Axiom R2, S and T must be sequenced rather than divergent. So either $\mathrm{S}$ precedes $\mathrm{T}$ or $\mathrm{T}$ precedes $\mathrm{S}$. If $\mathrm{T}$ precedes $\mathrm{S}$, then by Axiom R3, there exists a refinement $\mathrm{T}^{*}{ }_{2}$ of $\mathrm{T}$ that precedes $\mathrm{S}^{*}$ and hence $\mathrm{T}^{*}$; since $\rho(\mathrm{T})$ is a clade, this implies that $\mathrm{T}^{*}{ }_{2}=\mathrm{S}^{*}$ $=\mathrm{T}^{*}$; and so by Axiom R1, $\mathrm{S}=\mathrm{T}$. Hence $\mathrm{S}$ precedes $\mathrm{T}$.

7. Recall that (1) $\Phi$ containing a cut of $\Xi$ is equivalent to $\Xi$ foretells $\Phi$ and (2) $\Xi$ foretells $\Phi$ is equivalent to $S$ foretells $\Phi$ for all $S$ in $\Xi$. Using these equivalences and Statement 4 , we see that $\Phi$ containing a cut of $S$ is equivalent to $S$ foretells $\Phi$, which is equivalent to $\rho($ S) foretells $\rho(\Phi)$, which is equivalent to $\rho(\Phi)$ containing a cut of $\rho(S)$, which is equivalent to $\rho(\Phi)$ containing a cut of $S^{*}$ for every $S^{*}$ in $\rho(S)$.

8. Let $\mathrm{S}$ and $\mathrm{T}$ be the situations refined by $\mathrm{S}^{*}$ and $\mathrm{T}^{*}$, respectively. By Statement 6 , S precedes T. By Proposition 5, T can be extended to a cut $\Phi$ of S. By Statement 7, $\rho(\Phi)$ contains a cut of $S^{*}$. Since $T^{*}$ is a successor of $S^{*}$ and is in $\rho(\Phi)$, the maximality of the cut requires that it contain $\mathrm{T}^{*}$.

9. In order to show that $\Gamma^{*} 0$ is subordinate to $\Gamma^{*}$, it suffices to consider a path $\omega$ in $\Gamma^{*}$ and to show that $\omega \cap \Gamma^{*}{ }_{0}$ is a path in $\Gamma^{*} 0$. To show that $\omega \cap \Gamma^{*}{ }_{0}$ is a path in $\Gamma^{*} 0$, it suffices to consider a situation $S^{*}$ in $\Gamma^{*} 0$ that is sequenced with all the situations in $\omega \cap \Gamma^{*} 0$ and to show that it is in $\omega$. We write $S$ for the situation in $\Gamma$ that $S^{*}$ refines, and we consider two cases: (1) $\mathrm{S}^{*}$ has a successor in $\omega \cap \Gamma^{*}{ }_{0}$, and (2) $\mathrm{S}^{*}$ has no successor in $\omega \cap \Gamma^{*} 0$. In case (1), $S^{*}$ is in $\omega$ because it has a successor in $\omega$. In case (2), $S^{*}$ is a successor of all the situations in $\omega \cap \Gamma^{*} 0$. By Statement $8, S^{*}$ can be extended to a cut consisting of situations in $\Gamma^{*} 0$. Choose such a cut, and let $\mathrm{T}^{*}$ be the situation it has in common with $\omega$. Then $T^{*}$ is a predecessor of $S^{*}$ and yet is in the same cut as $\mathrm{S}^{*}$. This means that $T^{*}=S^{*}$, so that $S^{*}$ is in $\omega$. 
Since the mapping $\rho$ is not altered, Axioms R0, R1, and R6 continue to hold. Omitting situations from an event tree does not alter any precedence relations for situations that remain. From this we can see first that it does not alter the set of prerequisite relations or the set of allows relations among clades that remain, then that it does not alter the set of precedes relations, and then that it does not alter the set of divergent relations. Hence Axioms R2, R3, and R4 continue to hold under $\rho_{0}$.

The case of implies relations is slightly more subtle. In general, omitting situations from an event tree cannot destroy implies relations, but it can introduce new ones. So we must consider the case where $\rho(\Xi)$ implies $\rho(\Phi)$ does not hold in $\Gamma^{*}$. Is it possible that when we omit all the situations that do not refine situations in $\Gamma$, we omit all the successors of $\rho(\Xi)$ divergent from $\rho(\Phi)$, so that $\rho(\Xi)$ implies $\rho(\Phi)$ holds in $\Gamma^{*}$ ? No. Since $\Xi$ implies $\Phi$ does not hold in $\Gamma$, there is a successor T of $\Xi$ that is divergent from $\Phi$. Any refinement $T^{*}$ of T will be a successor of $\rho(\Xi)$ divergent from $\rho(\Phi)$.

10. Statement 10 follows from Axiom R3.

11. Statement 11 follows from Axiom R4.

12. By Statement 6 , any two situations in $\rho^{-1}\left(\omega^{*}\right)$ are sequenced. So in order to show that $\rho^{-1}\left(\omega^{*}\right)$ is a path from $S$, it suffices to consider a successor $T$ of $S$ that is sequenced with all the situations in $\rho^{-1}\left(\omega^{*}\right)$ and show that $\mathrm{T}$ is in $\rho^{-1}\left(\omega^{*}\right)$. We consider two cases. (1) T has a successor $\mathrm{U}$ in $\rho^{-1}\left(\omega^{*}\right)$. Let $\mathrm{U}^{*}$ be $\mathrm{U}$ 's refinement in $\omega^{*}$. Statement 10 tells us that $\mathrm{T}$ has a refinement $\mathrm{T}^{*}$ that precedes $\mathrm{U}^{*}$ and hence is also in $\omega^{*}$. So $\mathrm{T}$ is in $\rho^{-1}\left(\omega^{*}\right)$. (2) T does not have a successor in $\rho^{-1}\left(\omega^{*}\right)$; all the situations in $\rho^{-1}\left(\omega^{*}\right)$ precede T. In this case, choose a cut $\Phi$ of S containing T. By Statement 7, $\rho(\Phi)$ contains a cut of $S^{*}$, and hence the path $\omega^{*}$ goes through $\rho(\Phi) ; \rho^{-1}\left(\omega^{*}\right)$ and $\Phi$ have a situation in common. Since T is sequenced with all the situations in $\rho^{-1}\left(\omega^{*}\right)$ and 
divergent from all the other situations in $\Phi$, none of the other situations in $\Phi$ can be in $\rho^{-1}\left(\omega^{*}\right)$. So T is in $\rho^{-1}\left(\omega^{*}\right)$.

Now suppose $\omega$ is a path from S. Choose a situation $\mathrm{T}$ in $\omega$. Using Statement 11, choose a situation $\mathrm{T}^{*}$ that follows $\mathrm{S}^{*}$ and refines $\mathrm{T}$. Let $\omega^{*} 0$ be the predecessors of $\mathrm{T}^{*}$. Then $\omega^{*} 0$ is a set of sequenced successors of $S^{*}$, and by Statements 6 and $10, \rho^{-1}\left(\omega^{*}\right)$ is the subset of $\omega$ consisting of all situations that follow $\mathrm{S}$ and precede T. For any successor $\mathrm{T}_{1}$ of $\mathrm{T}$ in $\omega$, we can repeat this exercise, extending $\omega^{*} 0$ to a set $\omega^{*}{ }_{1}$ of sequenced successors of $S^{*}$ that includes a refinement of $T_{1}$, so that $\rho^{-1}\left(\omega^{*} 1\right)$ is the subset of $\omega$ consisting of all situations that follow $\mathrm{S}$ and precede $\mathrm{T}_{1}$. By the axiom of choice, we can enlarge $\omega^{*} 0$ to a set $\omega^{*} \infty$ of sequenced successors of $S^{*}$ such that $\rho^{-1}\left(\omega^{*} \infty\right)=\omega$. Again by the axiom of choice, we can enlarge $\omega_{\infty}^{*}$ to a path $\omega^{*}$ from $S^{*}$, since this will only involve adding successors that do not refine situations in $\Gamma$, we will have $\rho^{-1}\left(\omega^{*}\right)=\omega$.

13. By Statement 7, $\rho(\Phi)$ contains a cut of $S^{*}$. Let $\Phi^{*}$ be this cut. In order to show that $\Phi=\rho^{-1}\left(\Phi^{*}\right)$, we must show that $\Phi^{*}$ contains a refinement of an arbitrary situation $\mathrm{T}$ in $\Phi$. There is a path from $S$ through $T$, and by Statement 12 , this path is equal to $\rho^{-1}\left(\omega^{*}\right)$ for some path from $\mathrm{S}^{*}$. Let $\mathrm{T}_{1} *$ be the refinement of $\mathrm{T}$ in $\omega^{*}$, and let $\mathrm{T}_{2} *$ be the situation where $\Phi^{*}$ cuts $\omega^{*}$. Since they are both in $\omega^{*}, \mathrm{~T}_{1}^{*}$ and $\mathrm{T}_{2} *$ are sequenced. Since they are both in $\rho(\Phi)$, they must be divergent or equal. So they are equal; $\Phi^{*}$ contains a refinement of $\mathrm{T}$. 


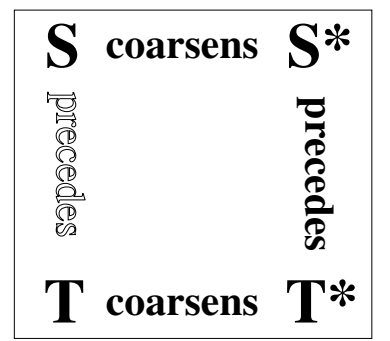

If the relations in bold hold, then the relation in outline holds.

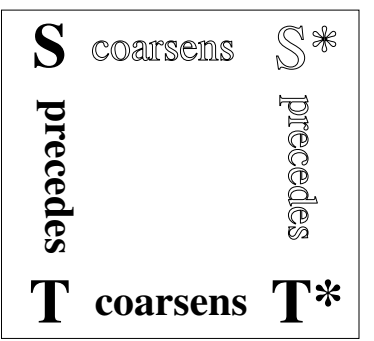

If the relations in bold hold, then there exists $\mathrm{S}^{*}$ such that the relations in outline hold.

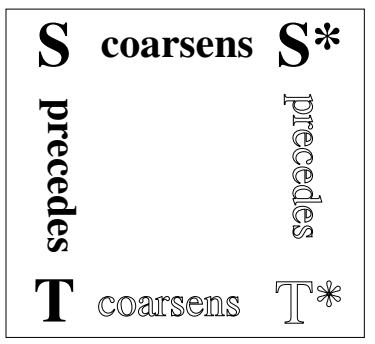

If the relations in bold hold, then there exists $\mathrm{T}^{*}$ such that the relations in outline hold.

Figure 27 Statements 6, 10, and 11 of Proposition 12.

5.2. An Alternative Axiomatization. Axioms R0-R6 constitute only one way of axiomatizing refinement; there are many others, some much more austere. For example, instead of thinking of refinement from $\Gamma$ to $\Gamma^{*}$ as a mapping that assigns a clade in $\Gamma^{*}$ to each clade in $\Gamma$, we can step back to thinking of it as a mapping that assigns a clade of situations $\rho(S)$ in $\Gamma^{*}$ to each situation $S$ in $\Gamma$, and we can adopt the following axioms:

Axiom R0' $\rho(\mathrm{I})=\left\{\mathrm{I}^{*}\right\}$.

Axiom R1' All the $\rho(\mathrm{S})$ are disjoint from each other.

Axiom R2' If $S$ divergent $T$, then $\rho(S)$ divergent $\rho(T)$.

Axiom R3' If $S$ precedes $T$, then $\rho(S)$ precedes $\rho(T)$.

Axiom R4' Suppose $\Phi$ is a clade in $\Gamma$ and $S^{*}$ is in $\rho(S)$. Then if $\Phi$ is a cut of $S$ if and only if $\cup_{T \in \Phi} \rho(T)$ contains a cut of $S^{*}$.

As the following proposition shows, Axioms R0'-R4' are equivalent to Axioms R0-R6.

\section{Proposition 13.}


1. Suppose $\rho$ is a mapping from clades in $\Gamma$ to clades in $\Gamma^{*}$ that satisfies Axioms $R 0$ R6. Define a new mapping $\rho$ from situations in $\Gamma$ to clades in $\Gamma^{*}$ by setting $\rho(S):=$ $\rho(\{S\})$. Then this new mapping $\rho$ satisfies Axioms $R O^{\prime}-R 4^{\prime}$.

2. Suppose $\rho$ is a mapping from situations in $\Gamma$ to clades in $\Gamma^{*}$ that satisfies Axioms $R 0^{\prime}-R 4^{\prime}$. Define a new mapping $\rho$ from clades in $\Gamma$ to sets of situations in $\Gamma^{*}$ by setting $\rho(\Xi):=\cup_{S \in \Xi} \rho(S)$. Then $\rho(\Xi)$ is a clade in $\Gamma^{*}$ for every clade $\Xi$ in $\Gamma$, and $\rho$ satisfies Axioms RO-R6.

Proof 1. Axiom R0' follows from Axiom R0. Axiom R1' follows from Axiom R1. Axiom R2' follows from Axiom R2. Axiom R3' follows Statement 3 of Proposition 12. Axiom R4' follows from Statement 7 of Proposition 12.

2. It follows from Axiom R2' that each $\rho(\Xi)$ is a clade. Axiom R0 follows from Axioms R0' and R1'. Axiom R1 follows from Axiom R1'.

It follows from Axiom R2' that if $\Xi$ is divergent from $\Phi$, then $\rho(\Xi)$ is divergent from $\rho(\Phi)$. On the other hand, if $\Xi$ is not divergent from $\Phi$, then

there are situations $S$ in $\Xi$ and $T$ in $\Phi$ that are sequenced, and hence, by Axiom R3', there are situations $S^{*}$ in $\rho(S)$ and $T^{*}$ in $\rho(T)$ that are sequenced, whence $\rho(\Xi)$ is divergent from $\rho(\Phi)$. This establishes Axiom R2.

Suppose $\Xi$ prerequisite $\Phi$. Consider a situation $\mathrm{T}^{*}$ in $\rho(\Phi)$. Let $\mathrm{T}$ be the situation in $\Phi$ that coarsens $\mathrm{T}^{*}$. By the assumption $\Xi$ prerequisite $\Phi$, T has a predecessor $\mathrm{S}$ in $\Xi$. By Axiom $\mathrm{R}^{\prime}, \mathrm{T}^{*}$ has a predecessor, say $\mathrm{S}^{*}$, in $\rho(\Xi)$. Since $\mathrm{T}^{*}$ was an arbitrary situation in $\rho(\Phi)$, this establishes that $\rho(\Xi)$ prerequisite $\rho(\Phi)$.

Now suppose $\rho(\Xi)$ prerequisite $\rho(\Phi)$. Consider a situation T in $\Phi$. Choose a situation $\mathrm{T}^{*}$ in $\rho(\mathrm{T})$. Let $\mathrm{S}^{*}$ be $\mathrm{T}^{*}$ 's predecessor in $\rho(\Xi)$, and let $\mathrm{S}$ be the situation in $\Xi$ that coarsens $\mathrm{S}^{*}$. By Axiom R2', $\mathrm{S}$ and $\mathrm{T}$ must be sequenced. If $\mathrm{T}$ were to precede $\mathrm{S}$, then by Axiom R3' we would have $\rho(T)$ precedes $\rho(S)$, which would imply the existence of a 
situation $T_{2} *$ in $\rho(T)$ such that $T_{2} *$ precedes $S^{*}$ precedes $T^{*}$, and since $\rho(T)$ is a clade, this would imply that $\mathrm{S}^{*}=\mathrm{T}^{*}$, whence $\left(\right.$ Axiom $\left.\mathrm{R} 1^{\prime}\right) \mathrm{S}=\mathrm{T}$. So $\mathrm{S}$ precedes $\mathrm{T}$. This establishes $\Xi$ prerequisite $\Phi$ and hence completes the derivation of Axiom R3.

Suppose $\Xi$ allows $\Phi$. Consider a situation $S^{*}$ in $\rho(\Xi)$. Let $S$ be the situation in $\Xi$ that coarsens $S^{*}$. Then $S$ has a successor $T$ in $\Phi$. By Axiom R3', $S^{*}$ has a successor, say $T^{*}$, in $\rho(\Phi)$. This establishes $\rho(\Xi)$ allows $\rho(\Phi)$.

Now suppose $\rho(\Xi)$ allows $\rho(\Phi)$. Consider a situation $S$ in $\Xi$. Choose a situation $S^{*}$ in $\rho(S)$. Let $T^{*}$ be a successor of $S^{*}$ in $\rho(\Phi)$, and let $\mathrm{T}$ be the situation in $\Phi$ that coarsens $\mathrm{T}^{*}$. By the same argument we used in the paragraph before last, $\mathrm{S}$ precedes $\mathrm{T}$. This establishes $\Xi$ allows $\Phi$ and hence completes the derivation of Axiom R4.

Suppose $\Xi$ implies $\Phi$. Consider a situation $S^{*}$ in $\rho(\Xi)$. Let $S$ be the situation in $\Xi$ that coarsens $S^{*}$. Then $S$ has either a predecessor or a cut in $\Phi$. If $S$ has a predecessor in $\Phi$, then by Axiom R3', $S^{*}$ has a predecessor in $\rho(\Phi)$. If $S$ has a cut in $\Phi$, then by Axiom R4', S* has a cut in $\rho(\Phi)$. This establishes that $\rho(\Xi)$ implies $\rho(\Phi)$.

Now suppose $\rho(\Xi)$ implies $\rho(\Phi)$. Consider a situation $S$ in $\Xi$. Choose a situation $S^{*}$ in $\rho(S)$. Then $S^{*}$ has either a predecessor or a cut in $\rho(\Phi)$. If $S^{*}$ has a predecessor in $\rho(\Phi)$, then by a previous argument, $S$ has a predecessor in $\Phi$. If $S^{*}$ has a cut in $\rho(\Phi)$, then by Axiom R4', S has a cut in $\Phi$. This establishes $\Xi$ implies $\Phi$ and hence completes the derivation of Axiom R5.

Finally, Axiom R6 follows from the definition $\rho(\Xi):=\cup_{S \in \Xi} \rho(S)$.

For further ways of axiomatizing refinement, see Section 13.3 of The Art of Causal Conjecture. 
5.3. Consistency with Finite Refinement. The following proposition assures us that the concept of refinement contained in Axioms R0-R6 agrees, in the finite case, with the concept of refinement developed in Section 2.

\section{Proposition 14.}

1. Suppose $\Gamma$ and $\Gamma^{*}$ are finite event trees, and $\Gamma^{*}$ is a refinement of $\Gamma$ in the sense of Section 2. For each situation $S$ in $\Gamma$, let $\rho(S)$ be the set of situations in $\Gamma^{*}$ that refine $S$. For each clade $\Xi$ in $\Gamma$, set

$$
\rho(\Xi)=\cup_{S \in \Xi} \rho(S)
$$

Then $\rho(\Xi)$ is always a clade in $\Gamma^{*}$, and the mapping $\rho$ satisfies Axioms R0-R6.

2. Suppose $\rho$ refines $\Gamma$ to $\Gamma^{*}$ in the sense of this section, and suppose $\Gamma$ and $\Gamma^{*}$ are finite. Then $\rho$ is a composition of elementary refinements of the kind described in Section 2.

Proof 1. Statement 1 is obviously satisfied by an elementary refinement. To see that it is also satisfied by a composition of elementary refinements, it suffices to notice that composition of refinements means composition of the corresponding mappings: if $\rho_{1}$ represents the refinement from $\Gamma$ to $\Gamma^{*}$, and $\rho_{2}$ represents the refinement from $\Gamma^{*}$ to $\Gamma^{* *}$, then $\rho_{2}{ }^{\circ} \rho_{1}$ represents the refinement from $\Gamma$ to $\Gamma^{* *}$. It is obvious from the form of the axioms that they are preserved by such composition.

2. We will prove Statement 2 using induction on the number of nonterminal situations in $\Gamma^{*}$. Suppose first that there is only one nonterminal situation in $\Gamma^{*}$, which is therefore the initial situation and has all the terminal situations as its daughters. And consider two cases: (1) there is no situation $S$ in $\Gamma$ such that $\rho(S)$ contains a terminal situation in $\Gamma^{*}$, and (2) there is such a situation $\mathrm{S}$. 
In case (1), $\rho$ maps every situation in $\Gamma$ to the clade $\left\{I^{*}\right\}$. But Axiom R0 tells us that $\rho$ maps only I to $\left\{I^{*}\right\}$. So I is the only situation in $\Gamma$, and the refinement is of the form shown in Figure 28. This is an elementary refinement.

\section{$I^{*}$}

I

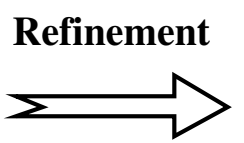

Figure 28 An elementary refinement, in which the original tree consists of a single situation, and the refined tree consists of a situation with daughters.

In case (2), S is distinct from I. By Axiom T0, S follows I. So by Statement 2 of Proposition 5, S is in a cut of I, say $\Xi$. By Axiom R5, $\rho(\Xi)$ is a cut of $I^{*}$, and since it is not equal to $\left\{I^{*}\right\}$, it must consist of all I*'s daughters, the terminal situations in $\Gamma^{*}$. So by Axioms R1 and R6, $\rho$ maps the situations in $\Xi$ to disjoint sets of these daughters. This establishes that $\rho$ is an elementary refinement of the type shown in Figure 29, provided that we can show that I and the situations in $\Xi$ are the only situations in $\Gamma$. But this is true by Axiom R2.

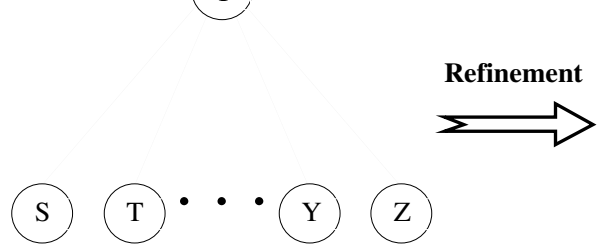

$I^{*}$

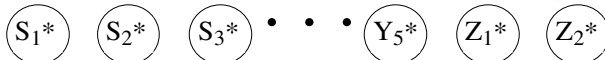

Figure 29 An elementary refinement, in which terminal situations are mapped to clades of terminal situations. 
Now suppose Statement 2 holds for refinements with n or fewer nonterminal situations, and suppose $\Gamma^{*}$ has $n+1$ nonterminal situations.

We consider three cases: (1) $\rho(S)$ is always a single situation, and every situation in $\Gamma^{*}$ refines a situation in $\Gamma,(2)$ at least one situation in $\Gamma^{*}$ does not refine a situation in $\Gamma$, and (3) every situation in $\Gamma^{*}$ refines a situation in $\Gamma$, and at least one pair of distinct situations in $\Gamma^{*}$ refine the same situation in $\Gamma$.

In case (1) $\rho$ maps $\Gamma$ one-to-one and onto $\Gamma^{*}$, and by Axiom R3 (or R4), it also preserves precedence, so that it is the identity mapping from the partially ordered set $\Gamma$ to itself, and this qualifies as an elementary refinement.

In case (2), choose a situation $Z^{*}$ in $\Gamma^{*}$ that does not refine a situation in $\Gamma$. Let $\mathrm{P}^{*}$ be the nearest predecessor of $Z^{*}$ that refines a situation in $\Gamma$. By Statement 8 of Proposition $8, \mathrm{P}^{*}$ either has no strict successors that refine situations in $\Gamma$ or has a whole cut of them. In the first case, we may omit the strict successors of $\mathrm{P}^{*}$ to obtain a smaller refinement of $\Gamma$, and the addition of the strict successors constitutes a further elementary refinement, so that the proof by induction is complete. In the second case we may choose the cut so that all the situations strictly between $\mathrm{P}^{*}$ and the cut do not refine elements of $\Gamma$, and $\mathrm{Z}^{*}$ will be one of these. Omitting these situations again produces a smaller refinement of $\Gamma$, and adding them back again constitutes a further elementary refinement.

Finally, consider case (3), where every situation in $\Gamma^{*}$ refines a situation in $\Gamma$, and at least one pair, say $\mathrm{S}_{1}{ }^{*}$ and $\mathrm{S}_{2}{ }^{*}$, refine the same situation $\mathrm{S}$. Then there must exist a pair of terminal situations, say $\mathrm{T}_{1}{ }^{*}$ and $\mathrm{T}_{2}{ }^{*}$, that refine the same situation $\mathrm{T}$. (Choose a terminal successor, say $\mathrm{T}_{1}$, of $\mathrm{S}_{1}{ }^{*}$, and let $\mathrm{T}$ be the situation it refines. By Statement 11 of Proposition 12, T is also terminal. By Statement 6 of Proposition 12, S precedes T, and 
by Statement 11 of Proposition 12, there exists $T_{2} *$ that also refines $T$ and follows $S_{2}{ }^{*}$. Since every situation in $\Gamma^{*}$ refines a situation in $\Gamma, \mathrm{T}_{2} *$ must also be terminal.)

Now consider any terminal situations $\mathrm{T}_{1} *$ and $\mathrm{T}_{2} *$ that refine the same situation $\mathrm{T}$. Let $\mathrm{S}^{*}$ designate the latest common predecessor of $\mathrm{T}_{1} *$ and $\mathrm{T}_{2} *$, and let $\mathrm{S}$ designate the situation refined by $\mathrm{S}^{*}$. Then $\mathrm{T}$ is terminal, and $\mathrm{S}$ precedes $\mathrm{T}$. Moreover, the situations in the closed interval $\left[\mathrm{S}^{*}, \mathrm{~T}_{1} *\right]$ and the situations in the closed interval $\left[\mathrm{S}^{*}, \mathrm{~T}_{1} *\right]$ both correspond one-to-one to the situations in the closed interval $[\mathrm{S}, \mathrm{T}]$. Let depth $\left(\mathrm{T}_{1}{ }^{*}, \mathrm{~T}_{2} *\right)$ denote the number of these situations.

From all the terminal situations that refine the same situation, choose a pair $\mathrm{T}_{1} *$ and $\mathrm{T}_{2} *$ with the least value of depth $\left(\mathrm{T}_{1} *, \mathrm{~T}_{2} *\right)$. Let $\mathrm{S} *$ designate their latest common predecessor. Let $\mathrm{R}_{1} *, \ldots, \mathrm{R}_{\mathrm{k}} *$ designate the daughters of $\mathrm{S}^{*}$, and let $\mathrm{R}_{1}, \ldots, \mathrm{R}_{\mathrm{k}}$ designate the situations they refine. We may assume that $\mathrm{R}_{1} *$ precedes $\mathrm{T}_{1} *$ and $\mathrm{R}_{2} *$ precedes $\mathrm{T}_{2} *$, so that $\mathrm{R}_{1}=\mathrm{R}_{2}$. By the assumption that depth $\left(\mathrm{T}_{1} *, \mathrm{~T}_{2} *\right)$ is minimal, all the terminals situations following a particular $\mathrm{R}_{\mathrm{i}} *$ refine distinct situations. We can therefore deduce from Axioms R3 and R4 that the subtree beginning at $\mathrm{R}_{\mathrm{i}} *$ is isomorphic to the subtree beginning at $\mathrm{R}_{\mathrm{i}}$, and hence that the subtrees beginning at $\mathrm{R}_{1} *$ and $\mathrm{R}_{2} *$ are isomorphic. If we remove one of these, say the subtree beginning at $\mathrm{R}_{1} *$, we will still have a refinement of $\Gamma$, and restoring it is an elementary refinement. This completes the inductive step of the proof.

\section{Event Spaces}

As we learned in Section 4, event trees pack a great deal of meaning into their apparent mathematical simplicity. There is information in the absence as well as in the presence of branches. This density of meaning is advantageous for probability theory, because it allows us to handle underlying logical relationships concisely and quickly, but 
it is not helpful when we want to couple probability ideas with explicit logical relationships or with information expressed linguistically.

After our study of refining, we can explain the limitations of event trees in another way: they do not permit the easy coexistence, in the same conversation, of situations described at different levels of detail. Two situations can coexist in the same event tree only if they give the same detail about all events up to the time of their happening.

In this section, we generalize from event trees to event spaces-spaces that can contain situations at many different levels of detail. Like an event tree, an event space describes possibilities for a single observer; each element is thought of as a situation she might find herself in, or alternatively, as the instantaneous event that she arrives in that situation. But unlike an event tree, an event space may contain two situations that can happen simultaneously, perhaps because one is simply a more detailed version of the other.

An event space can be thought of, as we shall see, as an event tree together with additional situations that represent directly the events or situations represented by clades in the tree. (Figure 30 provides an example.) But rather than define event spaces in terms of event trees, we provide a direct axiomatization for event spaces, designed to make clear their potential as a semantics for logics of action and causality. 

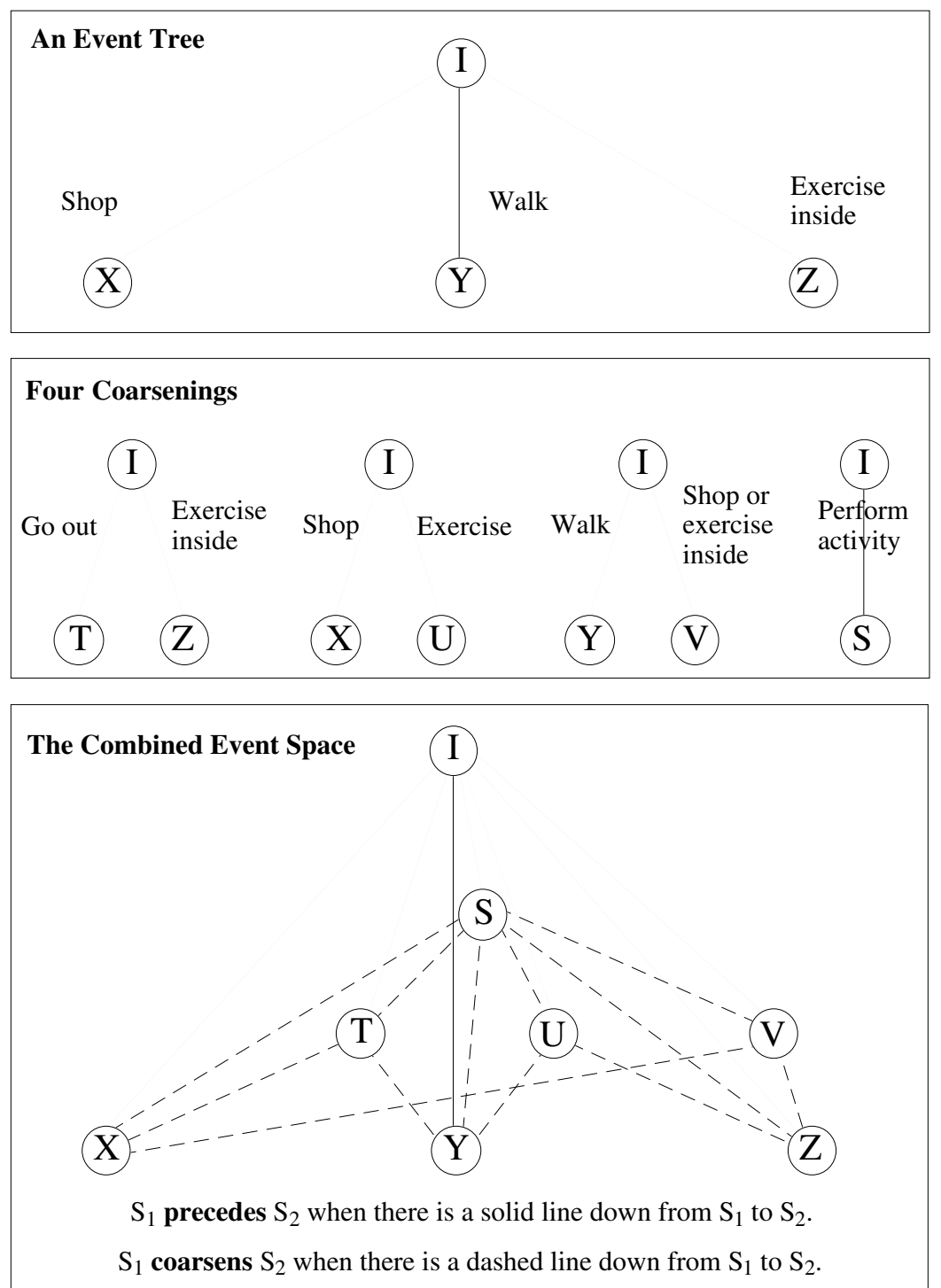

Figure 30 An event tree and an event space for a person who will soon begin to shop, walk, or exercise inside. The second panel shows four ways of simplifying the event tree; the first merges $\mathrm{X}$ and $\mathrm{Y}$ into $\mathrm{T}$, the second merges $\mathrm{Y}$ and $\mathrm{Z}$ into $\mathrm{U}$, the third merges $\mathrm{X}$ and $\mathrm{Z}$ into $\mathrm{V}$, and the fourth merges $\mathrm{X}, \mathrm{Y}$, and $\mathrm{Z}$ into $\mathrm{S}$. The event space, shown in the bottom panel, contains $\mathrm{X}, \mathrm{Y}$, and $\mathrm{Z}$, together with all the mergers. The two relations shown, precedes and coarsens, provide only partial information about how the event space's eight situations are related to each other. A complete description will be given in Table 2. 
6.1. Relations on an Event Space. Consider a set $\Gamma$, whose elements we call situations. In order to call a set $\Gamma$ an event space, we require the existence on $\Gamma$ of certain relations satisfying certain axioms. Before turning to the axioms, we list the relations. There are five primitive binary relations: overlaps, may.str.require, may.str.foretell, may.forbear, and may.diverge. We will define a large number of other relations, including relations with the same names and intuitive meanings as the event-tree relations we studied in Section 4, from these six primitive relations.

The meanings of the primitive binary relations are listed in Table 1 . We call these relations optional, because they say only that an event $\mathrm{S}$ may happen in a certain way.

\begin{tabular}{|lll|}
\hline \multicolumn{3}{|c|}{ THE PRIMARY OPTIONAL RELATIONS } \\
S 1 T & S overlaps T & S may happen in such a way that T happens at the \\
& same time. \\
S 2 T & S may.str.require T & S may happen in such a way that T has already \\
& & happened. \\
S 3 T & S may.str.foretell T & S may happen in such a way that T must happen \\
& & later. \\
S 4 T & S may.forbear T & S may happen in such a way that T remains only \\
& & possible. \\
S 5 T & S may.diverge T & S may happen in such a way that T cannot \\
happen. & &
\end{tabular}

Table 1 A number, a name, and a verbal explanation is given for each relation. The names all have English readings. For example, $\mathrm{S}$ may.str.require $\mathrm{T}$ is read "S may strictly require T." We read S may.diverge T as "S may diverge from T." 
In order to grasp the intuition underlying Table 1, the reader may wish to think about five ways a situation $S^{*}$ may relate to a clade $\Phi$ in an event tree. As Figure 31 shows, $S^{*}$ may be (1) in $\Phi$, (2) below $\Phi$, (3) above $\Phi$, with $\Phi$ containing a cut of it, (4) above $\Phi$, without $\Phi$ containing a cut of it, or (5) divergent from $\Phi$. If we identify the situations $S$ and $\mathrm{T}$ in our event space with clades $\Xi$ and $\Phi$ in an underlying event tree, then the situations $S^{*}$ in $\Xi$ are different ways $S$ may happen, and each relates to $\Phi$ in one of the five ways shown in the figure.

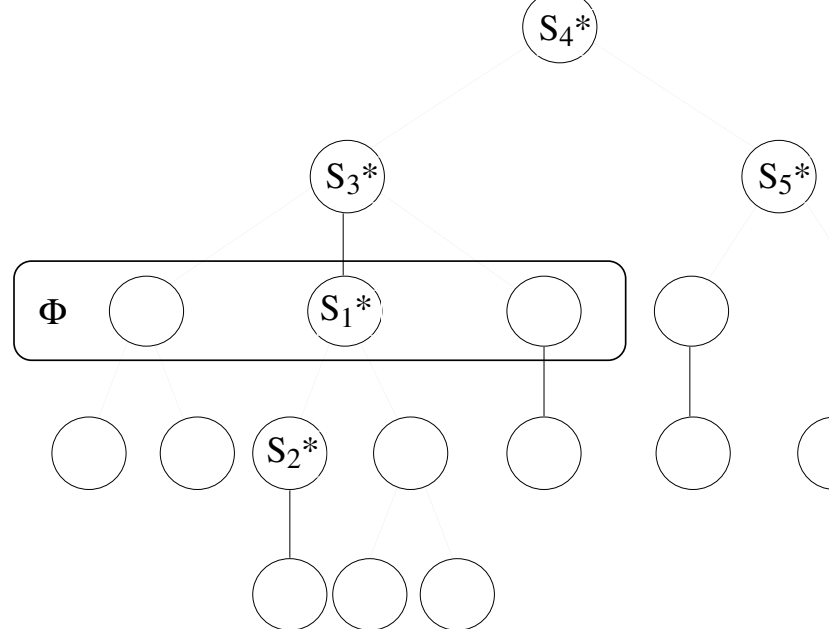

Figure 31 Five ways a situation $S^{*}$ can relate to a clade $\Phi$ in an event tree. (1) $S^{*}$ is in $\Phi$, and so $\Phi$ happens at the same time as $S^{*},(2) S^{*}$ is below $\Phi$, and so $\Phi$ has already happened when $S^{*}$ happens, (3) $S^{*}$ is above $\Phi$, and $\Phi$ contains a cut of $S^{*}$, and so $\Phi$ must happen after $S^{*}$ happens, (4) $S^{*}$ is above $\Phi$, but $\Phi$ does not contain a cut of $S^{*}$, and so $\Phi$ is only possible after $S^{*}$ happens, and (5) $S^{*}$ is divergent from $\Phi$, and so $\Phi$ cannot happen if $\mathrm{S}^{*}$ happens. 
It may also be helpful to spell out the five primary optional relations for the example in Figure 30. This is done in Table 2.

\begin{tabular}{|c|c|c|c|c|c|c|c|}
\hline \multirow[t]{7}{*}{ overlaps } & $(\mathrm{S}, \mathrm{S})$ & $(\mathrm{S}, \mathrm{T})$ & $(\mathrm{S}, \mathrm{U})$ & $(\mathrm{S}, \mathrm{V})$ & $(\mathrm{S}, \mathrm{X})$ & $(\mathrm{S}, \mathrm{Y})$ & $(\mathrm{S}, \mathrm{Z})$ \\
\hline & $(\mathrm{T}, \mathrm{S})$ & $(\mathrm{T}, \mathrm{T})$ & $(\mathrm{T}, \mathrm{U})$ & $(\mathrm{T}, \mathrm{V})$ & $(\mathrm{T}, \mathrm{X})$ & $(\mathrm{T}, \mathrm{Y})$ & \\
\hline & $(\mathrm{U}, \mathrm{S})$ & $(\mathrm{U}, \mathrm{T})$ & $(\mathrm{U}, \mathrm{U})$ & $(\mathrm{U}, \mathrm{V})$ & & $(\mathrm{U}, \mathrm{Y})$ & $(\mathrm{U}, \mathrm{Z})$ \\
\hline & $(\mathrm{V}, \mathrm{S})$ & $(\mathrm{V}, \mathrm{T})$ & $(\mathrm{V}, \mathrm{U})$ & $(\mathrm{V}, \mathrm{V})$ & $(\mathrm{V}, \mathrm{X})$ & & $(\mathrm{V}, \mathrm{Z})$ \\
\hline & $(\mathrm{X}, \mathrm{S})$ & $(\mathrm{X}, \mathrm{T})$ & & $(\mathrm{X}, \mathrm{V})$ & $(\mathrm{X}, \mathrm{X})$ & & \\
\hline & $(\mathrm{Y}, \mathrm{S})$ & $(\mathrm{Y}, \mathrm{T})$ & $(\mathrm{Y}, \mathrm{U})$ & & & $(\mathrm{Y}, \mathrm{Y})$ & \\
\hline & $(\mathrm{Z}, \mathrm{S})$ & & $(\mathrm{Z}, \mathrm{U})$ & $(\mathrm{Z}, \mathrm{V})$ & & & $(\mathrm{Z}, \mathrm{Z})$ \\
\hline may.str.require & $(\mathrm{S}, \mathrm{I})$ & $(\mathrm{T}, \mathrm{I})$ & $(\mathrm{U}, \mathrm{I})$ & $(\mathrm{V}, \mathrm{I})$ & $(\mathrm{X}, \mathrm{I})$ & $(\mathrm{Y}, \mathrm{I})$ & $(\mathrm{Z}, \mathrm{I})$ \\
\hline may.str.foretell & $(\mathrm{I}, \mathrm{S})$ & & & & & & \\
\hline may.forbear & & $(\mathrm{I}, \mathrm{T})$ & $(\mathrm{I}, \mathrm{U})$ & $(\mathrm{I}, \mathrm{V})$ & $(\mathrm{I}, \mathrm{X})$ & $(\mathrm{I}, \mathrm{Y})$ & $(\mathrm{I}, \mathrm{Z})$ \\
\hline \multirow[t]{6}{*}{ may.diverge } & & & $(\mathrm{T}, \mathrm{U})$ & $(\mathrm{T}, \mathrm{V})$ & $(\mathrm{T}, \mathrm{X})$ & $(\mathrm{T}, \mathrm{Y})$ & $(\mathrm{T}, \mathrm{Z})$ \\
\hline & & $(\mathrm{U}, \mathrm{T})$ & & $(\mathrm{U}, \mathrm{V})$ & $(\mathrm{U}, \mathrm{X})$ & $(\mathrm{U}, \mathrm{Y})$ & $(\mathrm{U}, \mathrm{Z})$ \\
\hline & & $(\mathrm{V}, \mathrm{T})$ & $(\mathrm{V}, \mathrm{U})$ & & $(\mathrm{V}, \mathrm{X})$ & $(\mathrm{V}, \mathrm{Y})$ & $(\mathrm{V}, \mathrm{Z})$ \\
\hline & & $(\mathrm{X}, \mathrm{T})$ & $(\mathrm{X}, \mathrm{U})$ & $(\mathrm{X}, \mathrm{V})$ & & $(\mathrm{X}, \mathrm{Y})$ & $(\mathrm{X}, \mathrm{Z})$ \\
\hline & & $(\mathrm{Y}, \mathrm{T})$ & $(\mathrm{Y}, \mathrm{U})$ & $(\mathrm{Y}, \mathrm{V})$ & $(\mathrm{Y}, \mathrm{X})$ & & $(\mathrm{Y}, \mathrm{Z})$ \\
\hline & & $(\mathrm{Z}, \mathrm{T})$ & $(\mathrm{Z}, \mathrm{U})$ & $(\mathrm{Z}, \mathrm{V})$ & $(\mathrm{Z}, \mathrm{X})$ & $(\mathrm{Z}, \mathrm{Y})$ & \\
\hline
\end{tabular}

Table 2 The primary optional relations for Figure 30. In contrast to the relations coarsens and precedes, which are shown in Figure 30, these relations describe fully how the event space's situations are related to each other.

The five primary optional relations can be used to form a large variety of further relations. For example, by forming disjunctions of primary optional relations, we get 
more optional relations. Since there are twenty-six ways of choosing two or more things from five, this provides us with twenty-six optional relations in addition to the primary ones. Table 3 lists and names a few of them.

\begin{tabular}{|c|c|c|}
\hline \multicolumn{3}{|c|}{ ADDITIONAL OPTIONAL RELATIONS } \\
\hline $\mathrm{S} 12 \mathrm{~T}$ & $S$ may.require $T$ & $\begin{array}{l}\text { S may happen in such a way that } \mathrm{T} \text { happens at the } \\
\text { same time or has already happened. }\end{array}$ \\
\hline $\mathrm{S} \mathbf{1 3} \mathrm{T}$ & S may.foretell T & $\begin{array}{l}\text { S may happen in such a way that } \mathrm{T} \text { happens at the } \\
\text { same time or must happen later. }\end{array}$ \\
\hline $\mathrm{S} \mathrm{34} \mathrm{T}$ & S may.str.allow $\mathrm{T}$ & $\begin{array}{l}\text { S may happen in such a way that } \mathrm{T} \text { must happen } \\
\text { later or remains only possible. }\end{array}$ \\
\hline $\mathrm{S} 45 \mathrm{~T}$ & $\mathrm{~S}$ may.forestall $\mathrm{T}$ & $\begin{array}{l}\mathrm{S} \text { may happen in such a way that } \mathrm{T} \text { remains only } \\
\text { possible or cannot happen. }\end{array}$ \\
\hline $\mathrm{S} 123 \mathrm{~T}$ & S may.imply $\mathrm{T}$ & $\begin{array}{l}\text { S may happen in such a way that } \mathrm{T} \text { happens at the } \\
\text { same time, has already happened, or must happen } \\
\text { later. }\end{array}$ \\
\hline $\mathrm{S} 134 \mathrm{~T}$ & $S$ may.allow $T$ & $\begin{array}{l}\text { S may happen in such a way that } \mathrm{T} \text { happens at the } \\
\text { same time, must happen later, or remains only } \\
\text { possible. }\end{array}$ \\
\hline $\mathrm{S} 1234 \mathrm{~T}$ & S compatible $\mathrm{T}$ & $\begin{array}{l}\mathrm{S} \text { may happen in such a way that } \mathrm{T} \text { happens at the } \\
\text { same time, has already happened, must happen } \\
\text { later, or remains only possible. }\end{array}$ \\
\hline
\end{tabular}

Table 3 The numbers for each relation list the primary relations whose disjunction it represents. For example, S $12 \mathrm{~T}$ means that either S $1 \mathrm{~T}$ or S $2 \mathrm{~T}$ (or both). The relation S compatible T is read "S is compatible with T." 
We can form yet further relations by negating optional relations. This produces mandatory relations, relations that restrict the way $\mathrm{S}$ can happen. Since there are thirtyone ways optional relations, there are also thirty-one mandatory relations. Table 4 lists, names, and explains a number of them. The names and explanations relay on the intuition that each time $\mathrm{S}$ happens, exactly one of the five possibilities shown in Figure 31 will hold for $\mathrm{T}$ - it happens at the same time, has already happened, must happen later, remains only possible, or cannot happen. Denying that some of these possibilities ever arise when $\mathrm{S}$ happens is the same as asserting that one of the remaining ones always does. Thus, for example, $\mathrm{S} \neg \mathbf{4 5} \mathrm{T}$ requires what $\mathrm{S} \mathbf{1 2 3} \mathrm{T}$ permits. More generally, if o is a proper subset of $\{1,2,3,4,5\}$ and (o) is its complement with respect to $\{1,2,3,4,5\}$, then $\mathrm{S} \neg(\mathbf{o}) \mathrm{T}$ requires what $\mathrm{S} \mathbf{o} \mathrm{T}$ permits. We will call $\neg(\mathbf{o})$ the mandatory relation corresponding to the optional relation $\mathbf{0}$. 


\begin{tabular}{|c|c|c|}
\hline \multicolumn{3}{|c|}{ PRIMARY MANDATORY RELATIONS } \\
\hline $\mathrm{S} \neg \mathbf{2 3 4 5} \mathrm{T}$ & $S$ refines $T$ & Whenever $\mathrm{S}$ happens, $\mathrm{T}$ happens at the same time. \\
\hline $\mathrm{S} \neg \mathbf{1 3 4 5} \mathrm{T}$ & $\mathrm{S}$ str.requires $\mathrm{T}$ & Whenever $\mathrm{S}$ happens, $\mathrm{T}$ has already happened. \\
\hline $\mathrm{S} \neg \mathbf{1 2 4 5} \mathrm{T}$ & $\mathrm{S}$ str.foretells $\mathrm{T}$ & Whenever $\mathrm{S}$ happens, $\mathrm{T}$ must happen later. \\
\hline $\mathrm{S} \neg \mathbf{1 2 3 5} \mathrm{T}$ & $\mathrm{S}$ forbears $\mathrm{T}$ & Whenever $\mathrm{S}$ happens, $\mathrm{T}$ remains only possible. \\
\hline $\mathrm{S} \neg \mathbf{1 2 3 4} \mathrm{T}$ & $S$ divergent $T$ & Whenever $\mathrm{S}$ happens, $\mathrm{T}$ cannot happen. \\
\hline \multicolumn{3}{|c|}{ ADDITIONAL MANDATORY RELATIONS } \\
\hline $\mathrm{S} \neg \mathbf{3 4 5} \mathrm{T}$ & $S$ requires $T$ & $\begin{array}{l}\text { Whenever } \mathrm{S} \text { happens, } \mathrm{T} \text { happens at the same time } \\
\text { or has already happened. }\end{array}$ \\
\hline $\mathrm{S} \neg 245 \mathrm{~T}$ & $\mathrm{~S}$ foretells $\mathrm{T}$ & $\begin{array}{l}\text { Whenever } \mathrm{S} \text { happens, } \mathrm{T} \text { happens at the same time } \\
\text { or must happen later. }\end{array}$ \\
\hline $\mathrm{S} \neg \mathbf{1 2 5} \mathrm{T}$ & S str.allows $\mathrm{T}$ & $\begin{array}{l}\text { Whenever } \mathrm{S} \text { happens, } \mathrm{T} \text { must happen later or } \\
\text { remains only possible. }\end{array}$ \\
\hline $\mathrm{S} \neg 123 \mathrm{~T}$ & $\mathrm{~S}$ forestalls $\mathrm{T}$ & $\begin{array}{l}\text { Whenever } \mathrm{S} \text { happens, T remains only possible or } \\
\text { cannot happen. }\end{array}$ \\
\hline $\mathrm{S} \neg \mathbf{4 5} \mathrm{T}$ & $\mathrm{S}$ implies $\mathrm{T}$ & $\begin{array}{l}\text { Whenever } \mathrm{S} \text { happens, } \mathrm{T} \text { happens at the same time, } \\
\text { has already happened, or must happen later. }\end{array}$ \\
\hline $\mathrm{S} \neg 25 \mathrm{~T}$ & $\mathrm{~S}$ allows $\mathrm{T}$ & $\begin{array}{l}\text { Whenever } \mathrm{S} \text { happens, } \mathrm{T} \text { happens at the same time, } \\
\text { must happen later, or remains only possible. }\end{array}$ \\
\hline $\mathrm{S} \neg \mathbf{5} \mathrm{T}$ & $\mathrm{S}$ tolerates $\mathrm{T}$ & $\begin{array}{l}\text { Whenever } \mathrm{S} \text { happens, } \mathrm{T} \text { happens at the same time, } \\
\text { has already happened, must happen later, or } \\
\text { remains only possible. }\end{array}$ \\
\hline
\end{tabular}

Table 4 Mandatory relations corresponding to the optional relations in Tables 1 and 3.

The numbers for each relation list the primary optional relations it denies. For example, $\mathrm{S} \neg 45 \mathrm{~T}$ means that S $45 \mathrm{~T}$ does not hold-i.e., that neither S $4 \mathrm{~T}$ nor S $5 \mathrm{~T}$ holds. 
(According to our terminology, however, $\mathrm{S} \neg \mathbf{4 5} \mathrm{T}$ corresponds to $\mathrm{S} \mathbf{1 2 3} \mathrm{T}$, not to the relation $\mathrm{S} 45 \mathrm{~T}$ that it denies.) We read $\mathrm{S}$ divergent $\mathrm{T}$ as " $\mathrm{S}$ is divergent from $\mathrm{T}$."

Table 5 shows the primary mandatory relations for Figure 30.

\begin{tabular}{|c|c|c|c|c|c|c|c|}
\hline \multirow[t]{5}{*}{ refines } & $(\mathrm{S}, \mathrm{S})$ & $(\mathrm{S}, \mathrm{T})$ & $(\mathrm{S}, \mathrm{U})$ & $(\mathrm{S}, \mathrm{V})$ & $(\mathrm{S}, \mathrm{X})$ & $(\mathrm{S}, \mathrm{Y})$ & $(\mathrm{S}, \mathrm{Z})$ \\
\hline & $(\mathrm{T}, \mathrm{S})$ & $(\mathrm{T}, \mathrm{T})$ & & & & & \\
\hline & $(\mathrm{U}, \mathrm{S})$ & & $(\mathrm{U}, \mathrm{U})$ & & & & \\
\hline & $(\mathrm{V}, \mathrm{S})$ & & & $(\mathrm{V}, \mathrm{V})$ & & & \\
\hline & & & & & $(\mathrm{X}, \mathrm{X})$ & $(\mathrm{Y}, \mathrm{Y})$ & $(\mathrm{Z}, \mathrm{Z})$ \\
\hline str.requires & $(\mathrm{S}, \mathrm{I})$ & $(\mathrm{T}, \mathrm{I})$ & $(\mathrm{U}, \mathrm{I})$ & $(\mathrm{V}, \mathrm{I})$ & $(\mathrm{X}, \mathrm{I})$ & $(\mathrm{Y}, \mathrm{I})$ & $(\mathrm{Z}, \mathrm{I})$ \\
\hline str.foretells & $(\mathrm{I}, \mathrm{S})$ & & & & & & \\
\hline forbears & & $(\mathrm{I}, \mathrm{T})$ & $(\mathrm{I}, \mathrm{U})$ & $(\mathrm{I}, \mathrm{V})$ & $(\mathrm{I}, \mathrm{X})$ & $(\mathrm{I}, \mathrm{Y})$ & $(\mathrm{I}, \mathrm{Z})$ \\
\hline \multirow[t]{4}{*}{ divergent } & & & & & $(\mathrm{U}, \mathrm{X})$ & $(\mathrm{V}, \mathrm{Y})$ & $(\mathrm{T}, \mathrm{Z})$ \\
\hline & & & $(\mathrm{X}, \mathrm{U})$ & & & $(\mathrm{X}, \mathrm{Y})$ & $(\mathrm{X}, \mathrm{Z})$ \\
\hline & & & & $(\mathrm{Y}, \mathrm{V})$ & $(\mathrm{Y}, \mathrm{X})$ & & $(\mathrm{Y}, \mathrm{Z})$ \\
\hline & & $(\mathrm{Z}, \mathrm{T})$ & & & $(\mathrm{Z}, \mathrm{X})$ & $(\mathrm{Z}, \mathrm{Y})$ & \\
\hline
\end{tabular}

Table 5 The primary mandatory relations for Figure 30.

Table 6 lists some further derived relations. The first two of these, precedes and alw.foretells, are more complex than the relations in Tables 1, 3, and 4, because they each combine relations going in the two directions. 


\begin{tabular}{|lll|}
\hline & \multicolumn{2}{c|}{ FURTHER DERIVED RELATIONS } \\
\hline RELATION & DEFINITION & ENGLISH READING \\
S precedes T & S allows T and T requires S & S precedes T \\
S alw.foretells T & S foretells T and T requires S & S always foretells T \\
S follows T & T precedes $\mathrm{S}$ & S follows T \\
S prerequisite T & T requires $\mathrm{S}$ & S is a prerequisite for T \\
S coarsens T & T refines $S$ & S coarsens T \\
\hline
\end{tabular}

Table 6 Definitions of some additional binary relations. The reader may wish to verify, using the definitions that of precedes and coarsens given here, that the specification of these relations in Figure 6.1 agrees with the specification of the primary optional relations in Table 2.

6.2. Axioms for Event Spaces. Now we list the axioms that the relations on $\Gamma$ must satisfy in order for $\Gamma$ to be called an event space. These axioms include a construction axiom for each of the six primitive relations.

Axioms S1 (Properties of individual binary relations.)
a. refines is asymmetric.
b. refines is reflexive.

Axioms S2 (Dependencies among binary relations.)
a. S may.str.require $\mathrm{T}$ if and only if $\mathrm{T}$ may.str.allow $\mathrm{S}$.
b. If $\mathrm{S}$ compatible $\mathrm{T}$ and $\mathrm{T}$ implies $\mathrm{U}$, then $\mathrm{S}$ compatible $\mathrm{U}$. 
Axiom S2a says that the second of the five primary optional relations can be defined in terms of the third and fourth. So in a sense we have only four primitive binary relations.

Axiom S3 (Refinement and the primary optional relations.) Suppose $\mathbf{i}$ is a primary optional relation. Then if $S^{*}$ refines $S$ and $S^{*} \mathbf{i} T$, then $S$ i T.

A clade is a nonempty set of situations, all of which are divergent from each other.

\section{Axioms S4 (Merger.)}

a. If $\Xi$ is a clade, then there is a situation, which we designate by $\oplus \Xi$, such that for every primary optional relation $\mathbf{i}, \oplus \Xi \mathbf{i}$ T if and only if $S * \mathbf{i}$ T for some $S^{*}$ in $\Xi$.

b. If $\Xi_{1}$ and $\Xi_{2}$ are divergent clades, then $\oplus\left(\Xi_{1} \cup \Xi_{2}\right)=\oplus\left(\left\{\oplus \Xi_{1}\right\} \cup \Xi_{2}\right)$.

c. If $\mathrm{U}$ and $\mathrm{V}$ are divergent from $\mathrm{T}$, and $\oplus\{\mathrm{T}, \mathrm{U}\}=\oplus\{\mathrm{T}, \mathrm{V}\}$, then $\mathrm{U}=\mathrm{V}$.

We call $\oplus \Xi$ the merger of $\Xi$, and we write $\oplus \Xi$ merges $\Xi$ and $\Xi$ decomposes $\oplus \Xi$. It follows from Axiom S4a and the asymmetry of refines (Axiom 1a) that $\oplus \Xi$ is the only situation decomposed by $\Xi$. In other words, if $\Xi$ decomposes $S$, then $S$ is the merger of $\Xi$. Axiom $\mathrm{S} 4 \mathrm{~b}$ says that the operation $\oplus$ is associative. If $\Xi$ decomposes $\mathrm{T}$ and $\mathrm{S}$ alw.foretells T, we say that $\Xi$ is a $c u t$ of $S$.

Axiom S5 (Constructions for the primary optional relations.)

Suppose $1 \leq \mathrm{i} \leq 5$ and $\mathrm{S} \mathbf{i}$. Then there is a situation, which we designate by $\mathbf{i}_{\mathrm{T}}(\mathrm{S})$, such that

(1) $\mathbf{i}_{\mathrm{T}}(\mathrm{S})$ refines $\mathrm{S}$, 
(2) $\mathbf{i}_{\mathrm{T}}(\mathrm{S}) \neg$ (i) $\mathrm{T}$, and

(3) if $U$ refines $S$ and $U \neg$ (i) $T$, then $U$ refines $i_{T}(S)$.

We write Overlap $(S)$ for $\mathbf{1}_{\mathrm{T}}(\mathrm{S}), \operatorname{After}_{\mathrm{T}}(\mathrm{S})$ for $\mathbf{2}_{\mathrm{T}}(\mathrm{S})$, Foretell $_{\mathrm{T}}(\mathrm{S})$ for $\mathbf{3}_{\mathrm{T}}(\mathrm{S})$, Forbear $(S)$ for $\mathbf{4}_{\mathrm{T}}(\mathrm{S})$, and Diverge $\mathrm{T}(\mathrm{S})$ for $\mathbf{5}_{\mathrm{T}}(\mathrm{S})$.

It follows from the asymmetry of refines that $\mathbf{i}_{\mathrm{T}}(\mathrm{S})$ is the unique situation that satisfies the conditions of Axiom S3. In other words, if $\mathrm{R}$ refines $\mathrm{S}, \mathrm{R} \neg(\mathrm{i}) \mathrm{T}$, and $\mathrm{U}$ refines $\mathrm{R}$ whenever $U$ refines $S$ and $U \neg$ (i) $T$, then $R=i_{T}(S)$. It then follows from the reflexivity of refines (Axiom 1b) that $S=i_{T}(S)$ if $S \neg(i) T$.

When $\mathrm{S}$ i T does not hold, we say $\mathbf{i}_{\mathrm{T}}(\mathrm{S})$ does not exist. (For example, when $\mathrm{S}$ overlaps T does not hold, Overlapt $(\mathrm{S})$ does not exist.) We write $\operatorname{Decomp}_{\mathrm{T}}(\mathrm{S})$ for the set consisting of those of the $\mathrm{i}_{\mathrm{T}}(\mathrm{S})$ that do exist.

\section{Axioms S6 (Recomposition.)}

a. $\operatorname{Decomp}_{\mathrm{T}}(\mathrm{S})$ is a clade.

b. $\mathrm{S}=\oplus \operatorname{Decomp}_{\mathrm{T}}(\mathrm{S})$.

Axiom S6a says two things about the situations $\mathbf{1}_{\mathrm{T}}(\mathrm{S}), \mathbf{2}_{\mathrm{T}}(\mathrm{S}), \mathbf{3}_{\mathrm{T}}(\mathrm{S}), \mathbf{4}_{\mathrm{T}}(\mathrm{S})$, and $\mathbf{5}_{\mathrm{T}}(\mathrm{S})$ : (1) at least one of them exists, meaning that at least one of the relations S 1 T, S 2 T, S 3 T, S 4 T, and S 5 T holds, and (2) those that exist are divergent from each other. 


\begin{tabular}{|ll|}
\hline NAME & ENGLISH READING \\
Overlap $_{\mathrm{T}}(\mathrm{S})$ & part of S overlapping T (or the overlap of S and $\mathrm{T}$ ) \\
$\operatorname{After}_{\mathrm{T}}(\mathrm{S})$ & part of S coming after T \\
Foretell $_{\mathrm{T}}(\mathrm{S})$ & part of S foretelling $\mathrm{T}$ \\
Forbear $_{\mathrm{T}}(\mathrm{S})$ & part of S forbearing T \\
$\operatorname{Diverge}_{\mathrm{T}}(\mathrm{S})$ & part of S diverging from $\mathrm{T}$ \\
$\oplus \Xi$ & merger of $\Xi$ \\
\hline
\end{tabular}

Table 7 English readings for the names of the six constructions.

6.3. Consequences. Now we work out some of the consequences of these definitions and axioms.

\section{Proposition 15.}

1. If $\mathrm{S}$ requires $\mathrm{T}$, then $\mathrm{S}$ implies $\mathrm{T}$.

2. If $S$ overlaps $T$, then $S$ compatible $T$.

3. If $\mathrm{S}$ allows $\mathrm{T}$, then $\mathrm{S}$ tolerates $\mathrm{T}$.

4. If $\mathrm{S}$ foretells $\mathrm{T}$, then $\mathrm{S}$ allows $\mathrm{T}$.

5. If $\mathrm{S}$ str.requires $\mathrm{T}$, then $\mathrm{S}$ requires $\mathrm{T}$.

6. If $\mathrm{S}$ str.foretells $\mathrm{T}$, then $\mathrm{S}$ foretells $\mathrm{T}$.

7. If $\mathrm{S}$ str.allows $\mathrm{T}$, then $\mathrm{S}$ allows $\mathrm{T}$.

Proof These statements follow directly from the definitions of the relations in terms of the primary optional relations.

\section{Proposition 16.}

1. At least one of the primary optional relations holds between $\mathrm{S}$ and $\mathrm{T}$. 
2. At most one of the primary mandatory relations holds between $\mathrm{S}$ and $\mathrm{T}$.

Proof As we noted earlier, Statement 1 is part of the content of Axiom S6a. Statement 2 follows because each primary mandatory relation denies all but one of the primary optional relations.

\section{Proposition 17.}

1. Suppose $\mathbf{i}$ is a primary optional relation. If $\mathrm{S} \neg$ (i) $\mathrm{T}$, then $\mathrm{S} \mathbf{i} \mathrm{T}$.

2. More generally, suppose $\mathbf{o}$ is an optional relation. If $\mathrm{S} \neg(\mathbf{o}) \mathrm{T}$, then $\mathrm{S} \mathbf{o} \mathrm{T}$.

3. Suppose $\mathrm{o}$ is an optional relation. If $\mathrm{S}^{*}$ refines $\mathrm{S}$ and $\mathrm{S}^{*} \mathrm{o} \mathrm{T}$, then $\mathrm{S} \boldsymbol{o} \mathrm{T}$.

4. Suppose $\mathbf{m}$ is a mandatory relation. If $S^{*}$ refines $S$ and $S \mathbf{m} T$, then $S^{*} \mathbf{m} T$.

5. Suppose $\mathrm{o}$ is an optional relation. If $\mathrm{S}^{*}$ refines $\mathrm{S}$ and $\mathrm{S}^{*} \neg(\mathbf{o}) \mathrm{T}$, then $\mathrm{S} \boldsymbol{o} \mathrm{T}$.

6. Suppose $\mathbf{i}$ is a primary optional relation. Then $\mathrm{S} \mathbf{i} \mathrm{T}$ if and only if there exists $\mathrm{S} *$ such that $\mathrm{S}^{*}$ refines $\mathrm{S}$ and $\mathrm{S}^{*} \neg$ (i) $\mathrm{T}$.

7. overlaps is reflexive.

8. divergent is irreflexive.

9. overlaps is symmetric, and if $S$ overlaps $T$, then $\operatorname{Overlap}_{T}(S)=\operatorname{Overlap}_{S}(T)$.

Proof 1. Statement 1 follows from Statement 1 of the preceding proposition.

2. Statement 2 follows from Statement 1.

3. Statement 3 follows from Axiom S3.

4. Statement 4 is the contrapositive of Statement 3.

5. Statement 5 follows from Statements 2 and 3.

6. Statement 6 follows from Statement 5 and Axiom S4.

7. Statement 7 follows from Statement 6 with $i=1$ and $S^{*}=S$.

8. Statement 8 follows form Statement 7, because $\mathrm{S}$ divergent $\mathrm{T}$ denies $\mathrm{S}$ overlaps $\mathrm{T}$. 
9. If $\mathrm{S}$ overlaps $\mathrm{T}$, Overlap $_{\mathrm{T}}(\mathrm{S})$ refines $\mathrm{T}$ and overlaps $\mathrm{S}$, and so by Axiom $\mathrm{S} 3$

T overlaps S. We know from Axiom S5 and the asymmetry of refines that Overlapt $(\mathrm{S})$ is the unique situation that refines both $\mathrm{S}$ and $\mathrm{T}$ and is refined by any other situation that also refines both $\mathrm{S}$ and $\mathrm{T}$. Since this condition is symmetric in $\mathrm{S}$ and $\mathrm{T}, \operatorname{Overlap}_{\mathrm{T}}(\mathrm{S})=$ Overlaps(T).

\section{Proposition 18.}

1. If $\mathrm{S}$ allows $\mathrm{T}$, then $\mathrm{S}$ compatible $\mathrm{T}$.

2. If $\mathrm{S}$ prerequisite $\mathrm{T}$ and $\mathrm{S}$ implies $\mathrm{T}$, then $\mathrm{S}$ allows $\mathrm{T}$.

3. $S$ precedes $T$ if and only if $S \neg 25 \mathrm{~T}$ and $\mathrm{T} \neg 5 \mathrm{~S}$.

4. $\mathrm{S}$ alw.foretells $\mathrm{T}$ if and only if $\mathrm{S} \neg \mathbf{2 4 5} \mathrm{T}$ and $\mathrm{T} \neg \mathbf{5} \mathrm{S}$.

5. 234 is symmetric.

6. compatible and divergent are symmetric.

7. Except for overlaps, the primary optional relations are irreflexive.

Proof Statement 1 follows from Statement 3 of Proposition 6.3.1 and Statement 2 of Proposition 6.3.3. Statements 2 through 5 follow from the definitions together with Axiom S2a. Statement 6 follows from Statement 5 together with Statement 9 of Proposition 6.3.3. Statement 7 follows from Statement 6.

Proposition 19. refines is transitive.

Proof This is the content of Statement 4 of Proposition 17 when $\mathbf{r}=$ refines.

Coupled with Axioms S1a and S1b, Proposition 19 says that refines is a partial ordering. If we agree to say that a given situation is the largest of a set of situations when it is refined by all the situations in that set, then we are now in a position to give a more 
colloquial explanation of the constructions in Axiom S5. We say that $\operatorname{Overlap}_{\mathrm{T}}(\mathrm{S})$ is the largest situation that refines both $\mathrm{S}$ and $\mathrm{T}, \operatorname{After} \mathrm{T}(\mathrm{S})$ is the largest situation that refines $\mathrm{S}$ and strictly requires $\mathrm{T}$, Foretell $\mathrm{s}_{\mathrm{T}}(\mathrm{S})$ is the largest situation that refines $\mathrm{S}$ and strictly foretells $\mathrm{T}$, Forbears $\mathrm{T}(\mathrm{S})$ is the largest situation that refines $\mathrm{S}$ and strictly forbears $\mathrm{T}$, and Divergent $\mathrm{T}(\mathrm{S})$ is the largest situation that refines $\mathrm{S}$ and is divergent from $\mathrm{T}$.

Proposition 20. Suppose $\Xi$ decomposes $\mathrm{S}$, and suppose $\mathrm{T}$ is a situation.

1. If $\mathbf{o}$ is an optional relation, then $S \mathbf{o} T$ if and only if $S^{*} \mathbf{o} T$ for some $S^{*} \in \Xi$.

2. If $\mathbf{m}$ is a mandatory relation, then $S \mathbf{m} T$ if and only if $S^{*} \mathbf{m} T$ for all $S^{*} \in \Xi$.

Proof These statements follow from Axiom S4a and the definitions of the optional and mandatory relations in terms of the primary optional relations.

\section{Proposition 21.}

1. If $\Xi$ decomposes $S$ and $S * E \Xi$, then $S *$ refines $S$.

2. $\left\{\mathrm{S}^{*}\right\}$ decomposes $\mathrm{S}$ if and only if $\mathrm{S}^{*}=\mathrm{S}$.

3. Suppose $T$ refines $S$, and $T \neq S$. Then $\{T$,Diverge $T(S)\}$ decomposes $S$.

Proof 1. Statement 1 follows from the reflexivity of refines and Statement 2 of Proposition 20.

2. By Statements 6 and 7 of Proposition $18, S=$ Overlap $_{T}(S)$, and $\mathbf{2}_{\mathrm{T}}(\mathrm{S}), \mathbf{3}_{\mathrm{T}}(\mathrm{S}), \mathbf{4}_{\mathrm{T}}(\mathrm{S})$, and $\mathbf{5}_{\mathrm{T}}(\mathrm{S})$ do not exist. So $\{\mathrm{S}\}$ decomposes $\mathrm{S}$. Similarly, $\left\{\mathrm{S}^{*}\right\}$ decomposes $\mathrm{S}^{*}$, so that if $\left\{S^{*}\right\}$ decomposes $S$, then $S=S^{*}$ by the uniqueness of merger.

3. The assumption $\mathrm{T}$ refines $\mathrm{S}$ means $\mathrm{T} \neg \mathbf{2 3 4 5} \mathrm{S}$, and by Axiom $\mathrm{S} 2 \mathrm{a}$, this implies $\mathrm{S} \neg \mathbf{2 3 4} \mathrm{T}$. Hence $\operatorname{After}_{\mathrm{T}}(\mathrm{S})$, Foretell $\mathrm{T}(\mathrm{S})$, and $\operatorname{Forbear}_{\mathrm{T}}(\mathrm{S})$ do not exist. So Decompt(S), which decomposes $\mathrm{S}$, consists of Overlap $\mathrm{T}(\mathrm{S})$, which is equal to $\mathrm{T}$, and $\operatorname{Diverge}_{\mathrm{T}}(\mathrm{S})$, if it 
exists. Since $\mathrm{T} \neq \mathrm{S}$, Statement 2 tells us that $\{\mathrm{T}\}$ cannot decompose $\mathrm{S}$ by itself. So $\operatorname{Diverge}_{\mathrm{T}}(\mathrm{S})$ does exist.

When $T$ refines $S$ and $T \neq S$, we sometimes write $S \backslash T$ for $\operatorname{Diverge}_{T}(S)$. We use the usual terminology of subtraction for this operation: $\mathrm{S} \backslash \mathrm{T}$ is the result of subtracting $\mathrm{T}$ from $S$.

Proposition 22. Suppose $\mathrm{T}$ refines $\mathrm{S}$ and $\mathrm{T} \neq \mathrm{S}$, so that $\operatorname{Diverge}_{\mathrm{T}}(\mathrm{S})$ exists.

1. Suppose $U$ refines $S$ and is divergent from $\operatorname{Diverge}_{T}(S)$. Then $U$ refines $T$.

2. $S \backslash(S \backslash T)=T$.

3. Suppose $U$ refines $S$ and is divergent from $T$. Then $U$ refines Diverge $T(S)$.

Proof 1 . We need to show that $\mathrm{U} \neg \mathbf{2 3 4 5} \mathrm{T}$. We will show first that $\mathrm{U} \neg \mathbf{2 3 4} \mathrm{T}$ and then that $\mathrm{U} \neg \mathbf{5} \mathrm{T}$.

The assumption that $\mathrm{U}$ refines $\mathrm{S}$ tells us that $\mathrm{U} \neg \mathbf{2 3 4} \mathrm{S}$, and by the symmetry of $\neg \mathbf{2 3 4}$ (Statement 5 of Proposition 18), this implies S $\neg \mathbf{2 3 4}$ U. By Statement 4 of Proposition 17, $\mathrm{T} \neg \mathbf{2 3 4} \mathrm{U}$, or $\mathrm{U} \neg \mathbf{2 3 4} \mathrm{T}$.

To show that $\mathrm{U} \neg \mathbf{5} \mathrm{T}$, we argue by contradiction. Suppose U $5 \mathrm{~T}$. Then DivergeU(T) refines $\mathrm{U}$ and is divergent from T. By Statement 4 of Proposition 17, Diverge $\mathrm{U}(\mathrm{T})$ is divergent from Diverge $\mathrm{T}(\mathrm{S})$. By Statement 4 of Proposition 21, Diverge $\mathrm{U}(\mathrm{T})$ is divergent from S. But by the transitivity of refines, DivergeU(T) refines S, and by Statement 2 of Proposition 16, this is a contradiction.

2. We need to show that $\mathrm{T}$ refines $\mathrm{S}$, is divergent from $\mathrm{S} \backslash \mathrm{T}$, and is refined by any other situation $\mathrm{U}$ that refines $\mathrm{S}$ and is divergent from $\mathrm{S} \backslash \mathrm{T}$. We are given that $\mathrm{T}$ refines $\mathrm{S}$. That $\mathrm{T}$ is divergent from $\mathrm{S} \backslash \mathrm{T}$ follows from the fact that $\mathrm{S} \backslash \mathrm{T}$ is divergent from $\mathrm{T}$. The final point is established by Statement 1. 
3. Statement 3 follows from Statements 1 and 2.

\section{Proposition 23.}

1. If $\Xi$ is a clade and $T \in \Xi$, then $\oplus(\Xi \backslash\{T\})=(\oplus \Xi) \backslash T$.

2. If we successively subtract members of a clade from the merger of the clade, the result is the merger of the clade obtained by removing those members.

Proof 1. By Axiom S5b, $\oplus \Xi=\oplus\{\oplus(\Xi \backslash\{T\}, T\}$. By Statement 3 of Proposition 21, $\oplus \Xi$ $\oplus \Xi=\left\{\operatorname{Diverge}_{\mathrm{T}}(\oplus \Xi), \mathrm{T}\right\}$. So by Axiom S4c, $\oplus\left(\Xi \backslash\{\mathrm{T}\}=\operatorname{Diverge}_{\mathrm{T}}(\oplus \Xi)\right.$. Statement 2 follows from Statement 1.

6.4. Subordination and Refining. The ideas of subordination and refinement, which were fairly complicated for event trees, are very simple for event spaces.

Suppose $\Gamma$ is an event space, and $\Gamma_{0}$ is a subset of $\Gamma$. And suppose $\Gamma_{0}$ includes all constructions from its situations. In other words, (1) if $S$ and $T$ are in $\Gamma_{0}$, then $\operatorname{Decomp}_{\mathrm{T}}(\mathrm{S})$ is a clade in $\Gamma_{0}$, and (2) if $\Xi$ is in $\Gamma_{0}$, then $\oplus \Xi$ is in $\Gamma_{0}$. Then obviously qualifies as an event space. It call it is subordinate to $\Gamma$.

Suppose $\Gamma$ and $\Gamma^{*}$ are event spaces, and suppose $\rho$ is a one-to-one mapping from $\Gamma$ to $\Gamma^{*}$ that preserves the primitive relations and the constructions. This means that

- $\quad \rho(S)$ i $\rho(T)$ if and only if $S$ i T, for $i=1,2,3,4,5$,

- $\oplus \rho(\Xi)=\rho(\oplus \Xi)$, and

- $\quad \mathbf{1}_{\rho(\mathrm{T})}(\rho(\mathrm{S}))=\rho\left(\mathbf{1}_{\mathrm{T}} \mathrm{S}\right)$.

Then we say that $\rho$ refines $\Gamma$ to $\Gamma^{*}$, and we say that $\Gamma^{*}$ is a refinement of $\Gamma$. If $\rho$ is onto, then we call $\rho$ an isomorphism. 
It is obvious that the image of a refining to $\Gamma^{*}$ is an event space subordinate to $\Gamma^{*}$.

6.5. Completeness. In contrast to an event tree, an event space expresses all its information in a positive way in its primitive relations and constructions. There is no further information conveyed in a negative way, by the absence of further situations. In this respect, the idea of an event space is more general than the idea of the set of clades of an event tree.

We say an event space $\Gamma$ is complete if whenever $\mathrm{S}$ precedes $\mathrm{T}$, there is a situation $\mathrm{U}$ such that $\mathrm{S}$ alw.foretells $\mathrm{U}$ and $\mathrm{T}$ refines $\mathrm{U}$.

An event space subordinate to a complete event space need not be complete. Figure 32 gives an example.

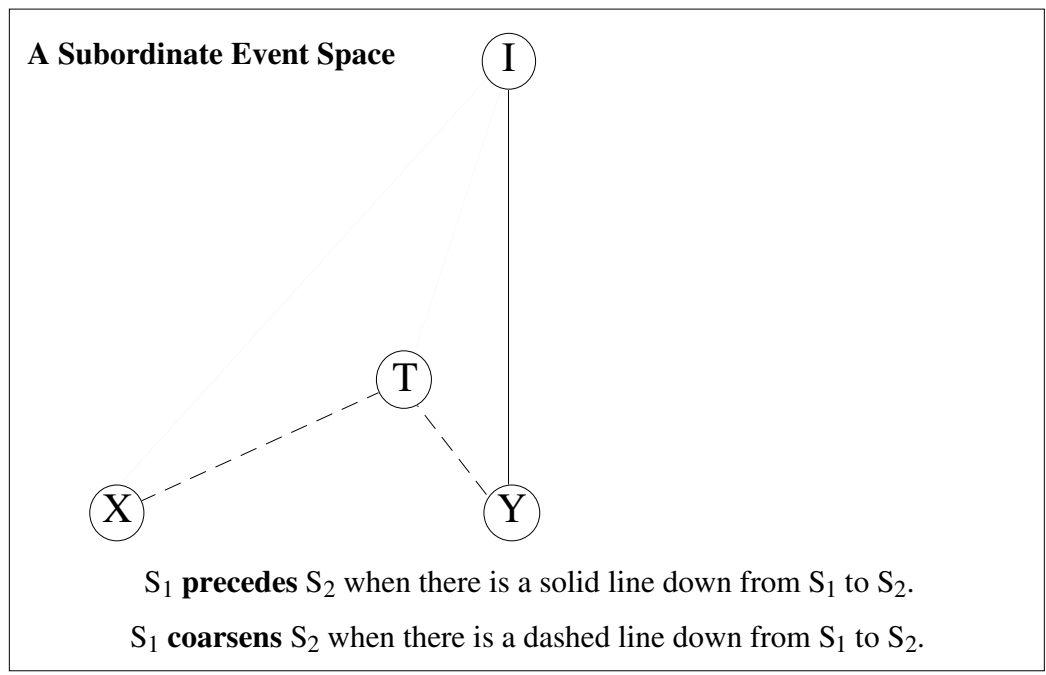

Figure 32 This event space, subordinate to the event space in Figure 30, is not complete. The situation I has several successors in the event space, but it has no cuts.

6.6. Relating Event Spaces to Event Trees. The introduction to this section explained that event spaces merely provide another way to think about event trees. An 
event space is basically the same as the a set of clades in an event tree. In this section we explain this point more fully.

Proposition 24. Suppose $\Gamma_{0}$ is an event tree. Let $\Gamma$ be the set consisting of the clades in $\Gamma_{0}$. Define relations overlaps, may.str.require, may.str.foretell, may.forbear, and may.diverge on $\Gamma$ as follows:

$S$ overlaps $T$ if $S \cap T \neq \varnothing$,

$S$ may.str.require $T$ if there are situations $S_{0} \in S$ and $T_{0} \in T$ such that $T_{0}$ precedes $_{0} S_{0}{ }^{6}$

$S$ may.str.foretell $T$ if there is a situation $S_{0}$ of $S$ such that $\left\{S_{0}\right\}$ foretells $0 T, 7$

$S$ may.forbear $T$ if there are situations $S_{0} \in S$ and $T_{0} \in T$ such that $S_{0}$ precedes $0 T_{0}$ but not $\left\{\mathrm{S}_{0}\right\}$ foretells $\mathrm{T}_{0}$.

$S$ may.diverge $T$ if there is a situation $S_{0} \in S$ such that $S_{0} \notin T$. Then $\Gamma$, with these as its primitive relations, is an event space. If $\Gamma_{0}$ is finite, then is complete.

We call the event space $\Gamma$ constructed in Proposition 24 the event space of clades in $\Gamma_{0}$.

Proposition 25. Every event space is isomorphic to an event space subordinate to the event space of clades from an event tree.

${ }^{6}$ Here precedes $_{0}$ is the precedence relation between situations in $\Gamma_{0}$.

${ }^{7}$ Here foretells ${ }_{0}$ is the relation foretells between clades in $\Gamma_{0}$, defined in Section 4. 


\section{GLENN SHAFER}

\section{References}

1. Dempster, A. P., Employment discrimination and statistical science (with discussion), Statistical Science, 2 (1988), 141-195.

2. Edwards, A.W.F., Pascal's Arithmetical Triangle, Charles Griffin, London, 1987.

3. Finkelstein, Michael O., and Bruce Levin, Statistics for Lawyers, Springer Verlag, New York, 1990.

4. Freedman, David A., Statistical models and shoe leather (with discussion), Sociological Methodology 21 (1991) 291-358.

5. Humphreys, Paul, The Chances of Explanation: Causal Explanation in the Social, Medical, and Physical Sciences, Princeton University Press, Princeton, New Jersey, 1989.

6. Jech, Thomas J., Set Theory, Academic Press, New York, 1978.

7. Kutschera, Franz von, Causation, Journal of Philosophical Logic 22 (1993) 563-588.

8. Raiffa, Howard, Decision Analysis, Addison-Wesley, Reading, Massachusetts, 1968.

9. Shafer, Glenn, Philosophical foundations for causal networks. Bernadette Bouchon-Meunier, Ronald R. Yager, and Lotfi A. Zadeh, eds., Advances in Intelligent Computing, Springer Verlag, New York, 1995, pp. 3-12.

10. Shafer, Glenn, The Art of Causal Conjecture. The MIT Press, Cambridge, Massachusetts, 1996.

11. Shafer, Glenn, The situation of causality. Submitted to Foundations of Science.

12. Shafer, Glenn, and Amos Tversky, Languages and designs for probability judgment, Cognitive Science 9 (1985) 305-339.

13. Vovk, Vladimir G., A logic of probability, with applications to the foundations of statistics (with discussion), Journal of the Royal Statistical Society, Series B 55 (1993a) 317-351.

14. Vovk, Vladimir G., Forecasting point and continuous processes: prequential analysis, Test 2 (1993b) 189-217.

\section{DEPARTMENT OF ACCOUNTING AND INFORMATION SYSTEMS, RUTGERS UNIVERSITY,} NEWARK, NEW JERSEY 07102

E-mail address: gshafer@andromeda.rutgers.edu

Home page: http://www.rutgers.edu/Accounting/raw/gsm/shafer.htm 